\title{
III. DIATOM PHYTOPLANKTON STUDIES IN THE SOUTHERN PACIFIC OCEAN, COMPOSITION AND CORRELATION TO THE ANTARCTIC CONVERGENCE AND ITS PALEOECOLOGICAL SIGNIFICANCE
}

\author{
Juliane Fenner, Hans-Joachim Schrader, and Harry Wienigk, Geologisch-Paläontologisches Institut \\ and Museum der Universität Kiel, D-23 Kiel, Federal Republic of Germany
}

\section{INTRODUCTION}

During Leg 35 of D/V Glomar Challenger from Callao, Peru to Ushuaia, Argentina, 2 February to 30 March 1974, surface water samples (4 m water depth) were collected in the southern part of the East Pacific Ocean, off the coast of Chile and in the Bellingshausen Sea. The study is supplementary to the investigation done on fossil diatoms of the sediment cores recovered during the leg (see Schrader, this volume).

Figures 1,2, and 3, and Table 1 show the position of the stations at which plankton collections and simultaneous surface water temperature measurements were made.

The object of the study was to determine: (1) patterns of the quantitative distribution of diatom species in the eastern South Pacific Ocean, with special emphasis on the distribution of Antarctic marine planktonic diatom species which are also found in sediment assemblages; (2) to provide information on the application of biometrical studies for reconstruction of past fluctuations in the general Antarctic circulation system.

\section{PREVIOUS STUDIES}

Diatoms are one of the most important constituents of the phytoplankton in the Southern Ocean and have been studied by many diatomists since Ehrenberg (1844) published the first paper on Antarctic diatoms. Taxonomy and distribution were studied by Castracane (1886), Van Heurck (1909), Heiden und Kolbe (1928), Peragallo (1921), Hart (1934), Hendey (1937), Hustedt (1958), Manguin (1960), Cassie (1963), Fukase and ElSayed (1965), Hargraves (1968), Hasle (1969), Donahue (1970), and Abbott (1972). (For a complete list see Kozlova, 1964, or Schrader, this volume.)

Quantitative studies became more numerous during the last 15 years. Hart (1942), Boden (1949), Kozlova (1964), and Hasle (1969) discussed relative and quantitative numbers of diatom species in sediment and water samples. From the distribution and frequency of diatom species it has been relatively simple to define the Subtropical Convergence (Hendey, 1937, Hart, 1942, Fukase, 1962, Kozlova, 1964, Hasle, 1969), whereas such has not been the case with the Antarctic Convergence.

Chlorophyll $a$ data have been measured and reported by Burkholder and Sieburth (1961), Mandelli and Burkholder (1965), El-Sayed and Mandelli (1965), and El-Sayed (1966a). Using the $\mathrm{C}_{14}$ technique for measurement of the primary production, Burkholder and Sieburth (1961) have observed a high phytoplankton biomass of up to $26.8 \mathrm{mgC} / \mathrm{m}^{3}$ in the inshore regions during the Antarctic summer. Although most chlorophyll data have been obtained from material collected during the Antarctic spring, summer, and autumn, Bunt (1961) and El-Sayed (1966b) made their observations also during the winter.

\section{OCEANOGRAPHY OF THE SOUTHEAST PACIFIC OCEAN}

The Southern Ocean (Deacon, 1963; The Oxford Atlas, 1963) is governed by the Westwind Drift resulting in the clockwise Antarctic Circumpolar Current which has volocities up to $25 \mathrm{~cm} / \mathrm{sec}^{-1}$ (Neumann and Pierson, 1966, cited in Gordon, 1971a) where west winds are at a maximum (at about $50^{\circ} \mathrm{S}$ latitude) with an average current velocity of $18 \mathrm{~cm} / \mathrm{sec}^{-1}$ (Kort, 1964). This zone of eastward water transport separates the warm subtropical and cold antarctic water masses and is limited in the north by the Subtropical Convergence (at about $40^{\circ} \mathrm{S}$ latitude) and in the south by the Antarctic Convergence (at about $60^{\circ} \mathrm{S}$ latitude). Between these two boundaries the temperature decreases from $17^{\circ}$ to $3^{\circ} \mathrm{C}$ and the salinity from $35^{\circ} \%$ to $34^{\circ} \%$ (Botnikov, 1964). Thus, three different water masses of the Southern Ocean exist. The Subtropical Convergence separates subtropic waters from the subantarctic water masses, whereas the Antarctic Convergence separates the subantarctic from the Antarctic water masses. At the latter boundary sinking occurs entraining some subantarctic Surface Water and Circumpolar Deep Water to produce the Antarctic Intermediate Water. The processes at the latter boundary can be much more complicated (Gordon, 1971a); even occasional signs of divergence have been observed (Wexler, 1959). The zone has been named the Antarctic Polar Front Gordon (1971b).

Close to the Antarctic continent, the Antarctic Divergence, a wind-produced feature, results in upwelling and addition of salty and warmer Circumpolar Deep Water, low in oxygen but high in nutrients, to the colder, highly oxygenated Antarctic Surface Water.

This configuration is partly destroyed, or at least complicated, in the study area where southernmost South America deflects most of the Subantarctic Waters to the north, forming the cold Peru Current, whereas the Antarctic Convergence is deflected to the south. Although the Antarctic Convergence is considered to be one of the most stable boundaries, impredictable loops, meanders, and eddies in it may occur, resulting in its shift southward or northward (Mackintosh, 1964).

The position of the Antarctic Convergence varies between $58^{\circ} \mathrm{S}$ and $63^{\circ} \mathrm{S}$ latitude (Deacon, 1973; Wyrtki, 


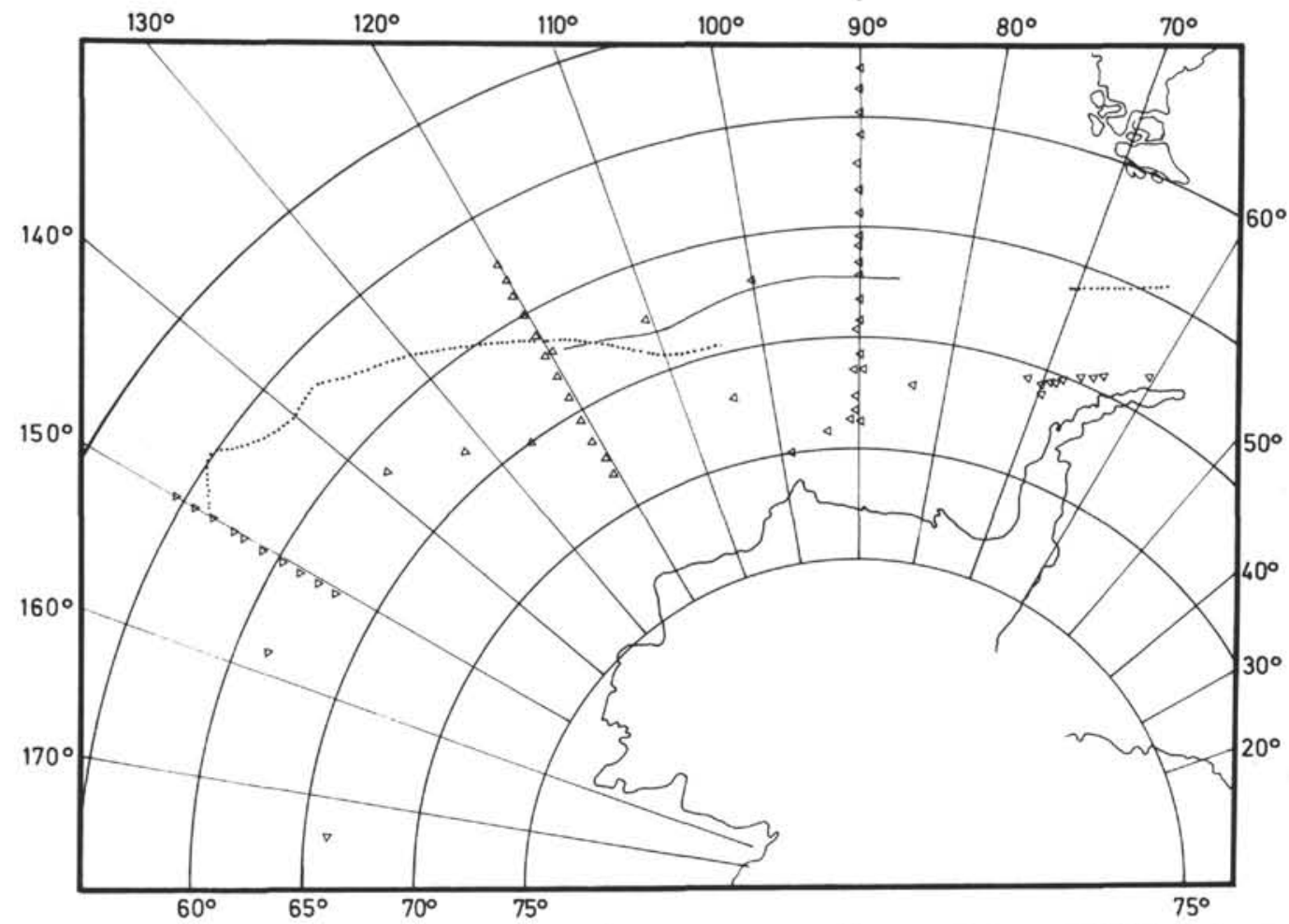

Figure 1. Location of Sections I (along $90^{\circ} \mathrm{W}$ ), II (along $120^{\circ} \mathrm{W}$ ), III (along $150^{\circ} \mathrm{W}$ ) of phytoplankton samples of the "Brategg" Expedition (Hasle, 1969).

1960; Ostapoff, 1962; Mackintosh, 1964; Gordon, 1967, $1971 \mathrm{~b}$ ) with its width being $2^{\circ}$ to $4^{\circ}$ of latitude (Gordon, 1967), or even as much as $5^{\circ}$ (Ostapoff, 1962). For the Antarctic Divergence the northern limit lies between $68^{\circ}$ to $70^{\circ} \mathrm{S}$ latitude (Deacon, 1937; Mackintosh, 1964).

\section{METHOD OF STUDY}

Collection of plankton samples, during both travel time and while on coring sites of Glomar Challenger, was accomplished by diverting water from the ship's water-cooling intake system (Figure 4) which is located about 4 meters below sea level beneath the ship's hull. Enroute to the laboratory the water passed through approximately 15 meters of pipe, a filter system of about 5 $\mathrm{mm}$ filter openings, and a turbine pump. In the laboratory it was filtered through a $22 \mu \mathrm{m}$ mesh phytoplankton net routinely every $2 \mathrm{hr}$ during travel time and sporadically while the ship was on coring station. Temperature readings, in degrees Fahrenheit, were measured at the seawater intake; they conformed closely to those made independently and routinely by the ship's weatherman.

At the time of collection the plankton was still alive and most zooplankton was undamaged. Collected samples were stored in glass vials in a $3 \%$ formaldehyde mixture. A 5-ml split of each plankton sample was oxydized by boiling it for $5 \mathrm{~min}$ in $50 \mathrm{ml}$ concentrated $\mathrm{HNO}_{3}$. Acid and soluble salts were removed from the samples by washing seven times in distilled water and centrifugation at $3000 \mathrm{rpm}$ for $5 \mathrm{~min}$. Splits of the cleaned samples were dried on a $18 \mathrm{~mm} \phi$ cover glass and mounted with Aroclor 4465, solvent xylene, refractive index of the final mixture 1.67 (Schrader, 1969).
Microscopic examination was performed with a Leitz Orthoplan/Orthomat photomicroscope with apochromatic optics, of maximum numerical aperture 1.32. The bulk of the photographs shown on the accompanying plates are at $1500 \times$ magnification; a few are at a lower magnification. Some of the photographs were made using a differential interference phase contrast setting. Counts were done with high-power oil immersion objective of $100 \times$, n.A. 1.32; counting all diatoms in traverses to about $300-400$ individuals. These were calculated to percent abundance within a sample, except for Chaetoceros bristles and fragments which were omitted from the countings. Counts are in valves and were done strictly afțer the method of Schrader (1974).

\section{RESULTS}

\section{Dominance and Latitudinal Distribution of Marine Diatoms in Antarctic Phytoplankton}

Results of the quantitative diatom analysis of the DSDP Leg 35 plankton samples are presented in Table 2. The latitudinal distribution and percent abundance of the more important species are presented in Figure 5 together with the respective temperature values of the stations. Two outstanding features are apparent in Figure 5: (1) the abrupt change from an extremely rich phytoplankton population south of $52^{\circ} \mathrm{S}$ latitude to the striking paucity of phytoplankton in the surface waters north of this position, and (2) the decrease or increase in population of certain species in direction of decreasing temperatures.

North of $52^{\circ} \mathrm{S}$ latitude the diatom population is completely different to that south of $52^{\circ}$. Coscinodiscus asteromphalus, Roperia tesselata, and Thalassiosira 


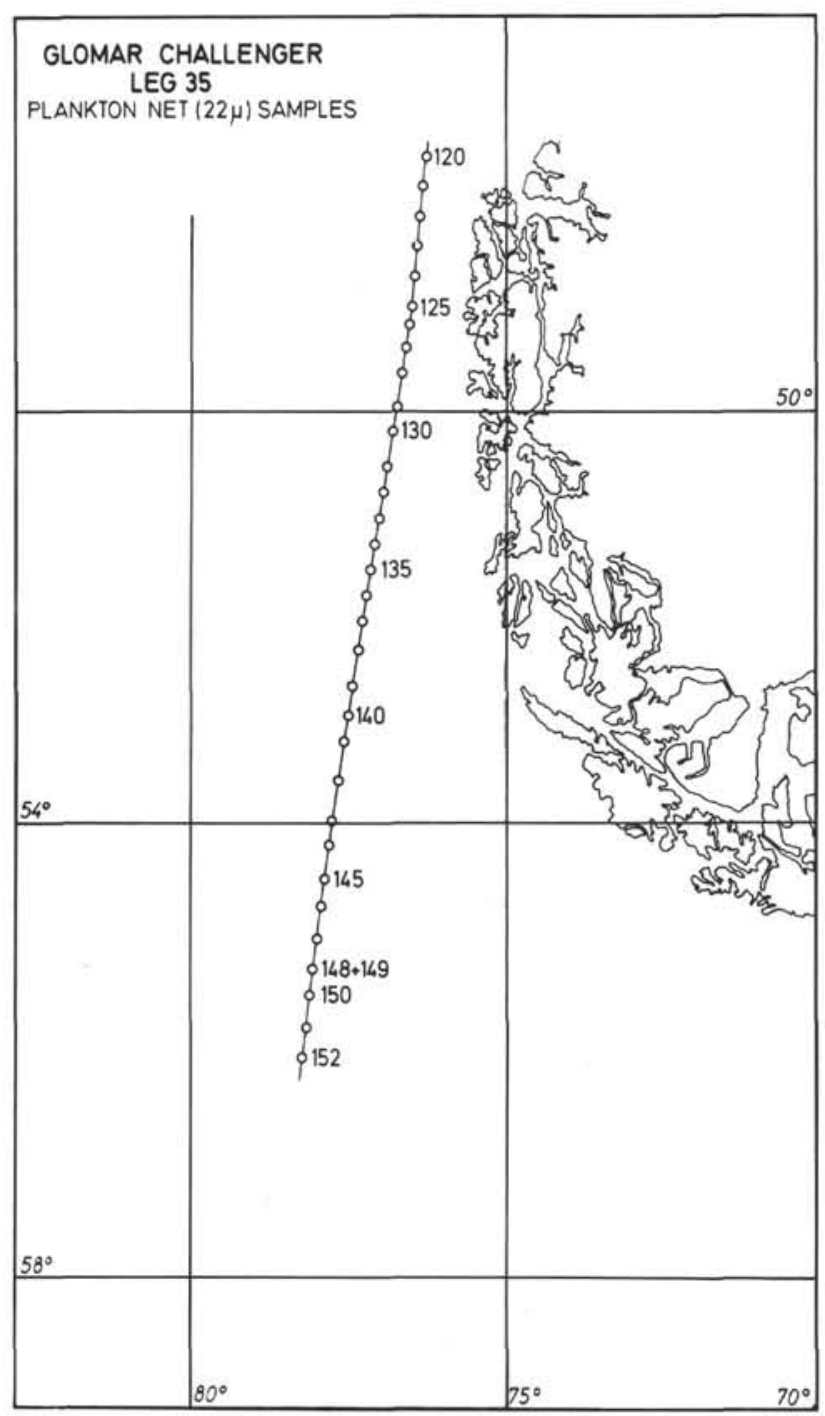

Figure 2. Location of phytoplankton stations during Leg 35 of $D / V$ Glomar Challenger (Stations 120-152).

poroseriata are the dominant species, accompanied by the sporadic occurrence of the predominantly tropical/subtropical to temperate species Actinocyclus curvatulus, A. ehrenbergii, A. ehrenbergii var. tenella, Actinoptychus splendens, Asteromphalus heptactis, Biddulphia longicruris, Coscinodiscus crenulatus, $C$. perforatus, C. radiatus, C. stellaris var. symbolophorus, Cyclotella stylorum, Hemidiscus cuneiformis, Lithodesmium undulatum, Rhizosolenia bergonii, Thalassiosira gravida, and $T$. lineata Jousé. By and large the samples were almost barren of diatoms and the above species occur only in extremely small numbers being far outnumbered by the crustaceans and dinoflagellates. Furthermore, most of the specimens in the region north of $52^{\circ} \mathrm{S}$ latitude are weakly silicified. The paucity of phytoplankton north of the Antarctic Convergence Zone was reported also by Hart (1934), Hendey (1937), and Boden (1949). It appears to be due to lack in nutrients and silica which have been depleted by the preceding phytoplankton bloom and have not been re- placed because of reduced vertical mixing, but because we do not have sufficient detailed oceanographic data on water masses of this area, we cannot make an exact interpretation of the abrupt change in the phytoplankton mass and species composition at $52^{\circ} \mathrm{S}$ latitude. Nor can we define a specific Subantarctic diatom assemblage, as Wood (1965) and Abbott (1972) did. The Subantarctic region in this study wherever diatoms were present in large quantities was dominated by Nitzschia kerguelensis. All other common species of the Antarctic were found in the Subantarctic waters as well, but most often in smaller numbers. A small percentage of temperate, or even tropical/subtropical species, from the north, are integrated into the flora with increasing quantity to the north towards the AAC, indicating that the Antarctic Convergence is not the barrier to diatom species distribution as it is for the zooplankton (Mackintosh, 1934, 1946; John, 1936; David, 1958; Baker, 1965). This study thus demonstrates that the Subantarctic zone is a region where mixing of Antarctic and temperate/subtropic diatom floras occurs and corroborate the results of Hendey (1937), Fukase (1962), Hargraves (1968), Hasle (1969), and Donahue (1972).

Figure 5 shows the predominance of Nitzschia kerguelensis in the entire area south of $52^{\circ} \mathrm{S}$ latitude, with only a slight reduction between $59^{\circ}$ and $63^{\circ} \mathrm{S}$ latitude (Antarctic Convergence), where other species such as Rhizosolenia and Nitschia angulata become more frequent. Nitzschia kerguelensis was found to never be outnumbered by Chaetoceros, Chorethron, or Rhizosolenia. As water temperature decreases toward Antarctica, Asteromphalus hyalinus, A. parvulus, Chaetoceros atlanticum f. bulbosum, Nitzschia curta, A. cylindrus, A. pseudonana, and Thalassiosira gracilis distinctly increase in numbers.

\section{Diversity of Marine Diatoms in Antarctic Phytoplankton}

Diversity calculations have been done on all counted DSDP Leg 35 phytoplankton samples; additional data were taken from Hasle (1969). The diversity index as defined by Berger and Parker (1970), is

$$
D_{H}=-\sum_{1}^{s} p_{i} \ln p_{i}
$$

wherein pi is the percent proportion of the individual species. The calculated diversity values shown in Figures 6 and 7, vary between 1 to 2.2, with two maxima, one between $60^{\circ}$ to $63^{\circ} \mathrm{S}$ latitude and the other one at the southernmost stations at $68^{\circ}$ to $69^{\circ} \mathrm{S}$ latitude. Two minima lie between $60^{\circ}$ to $53^{\circ} \mathrm{S}$ latitude, and between $68^{\circ}$ to $63^{\circ} \mathrm{S}$ latitude. Conversion and plotting of Hasle's (1969) data produced closely parallel results with a maximum between $60^{\circ}$ to $65^{\circ} \mathrm{S}$ latitude and a minimum between $55^{\circ}$ to $60^{\circ} \mathrm{S}$ latitude (Figure 7). Hasle placed the Antarctic Convergence in "Brategg" samples at approximately $63^{\circ} \mathrm{S}$ latitude for the section on $120^{\circ} \mathrm{W}$ longitude (Figure 7 , dotted line), and at approximately $63^{\circ} \mathrm{S}$ latitude for the section on $90^{\circ} \mathrm{W}$ 


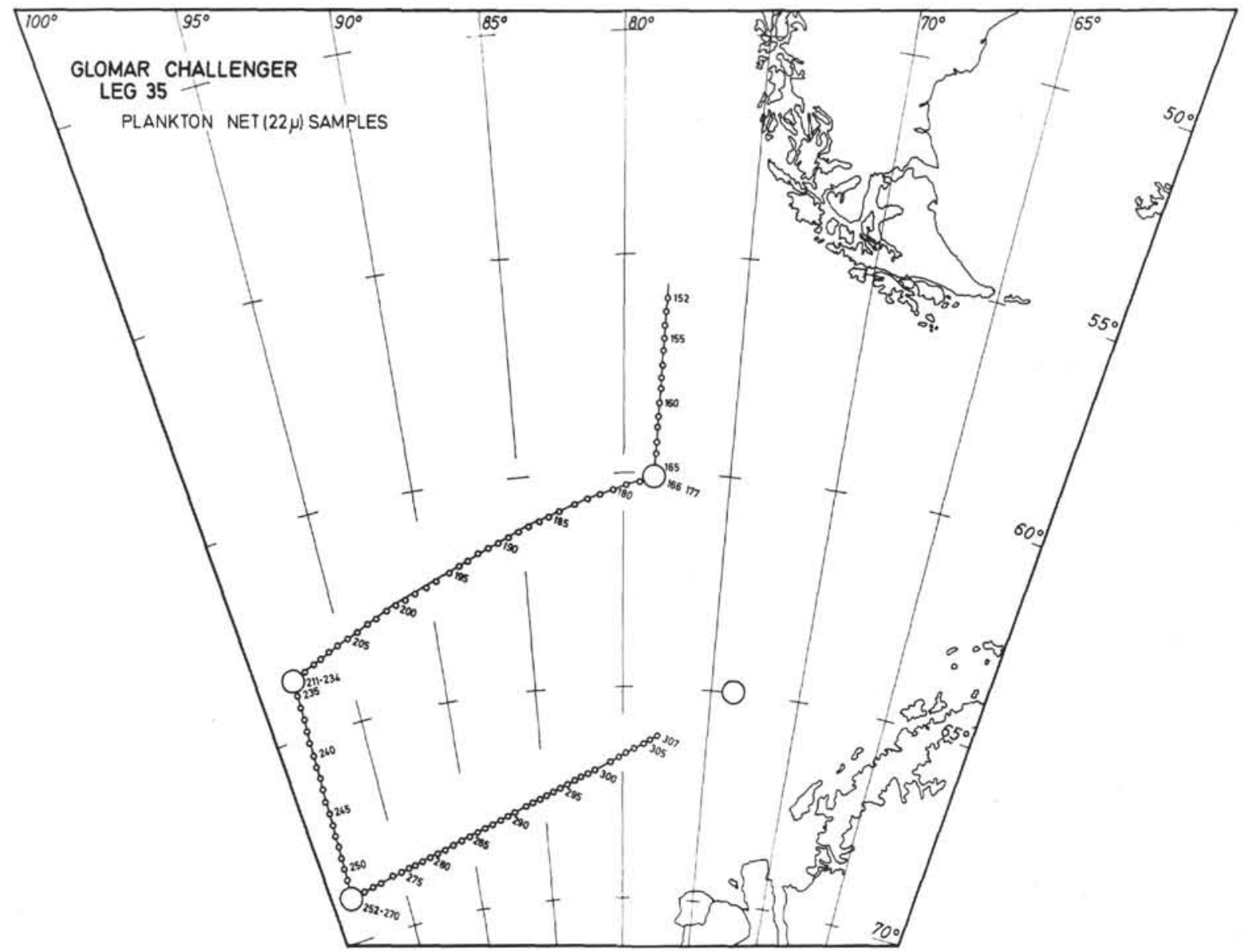

Figure 3. Location of phytoplankton stations during Leg 35 of D/V Glomar Challenger (Stations 152-307).

longitude (Figure 7, solid line). Hasle, using a more simplified index of diversity

$$
d=\frac{s-1}{\ln N}
$$

where $s$ is the number of species and $N$ the number of individuals, found her greatest values to be at station 1 $\left(52^{\circ} 50^{\prime} \mathrm{S}, 90^{\circ} \mathrm{W}\right)$.

Diversity values $(D H)$ for the two data sources are close analogs despite the samples on DSDP Leg 35 being collected during autumn (February-March, 1974) and those from the "Brategg" Expedition during summer (December-February, 1948). On the basis of temperature readings, the Antarctic Convergence was placed between $63^{\circ}$ to $60^{\circ} \mathrm{S}$ latitude in the eastern South Pacific, and the Antarctic Divergence, tentatively, between $68^{\circ}$ to $69^{\circ} \mathrm{S}$ latitude in the Bellingshausen Sea. These areas of diverging and converging water masses, with attendant extensive vertical mixing, appear to be characterized by a higher diversity of diatom population. Diversities of fossil populations have not been calculated because of the lack of well-preserved Quaternary assemblages. For this reason the authors are unable to demonstrate the applicability of diversity value of fossil assemblages for reconstruction of past shifts of
Antarctic circulation patterns. That the diversity was found to increase over nutrient-rich areas is somewhat surprising compared to situations found off northwest Africa and off Walvis Bay where lower diversity values were found in the upwelling areas compared to neighboring areas.

\section{Size Variations and Distribution Patterns of Two Diatom Species}

In order to test the intensity and kind of influence of hydrographic parameters on the size and morphology of diatoms, two common species Nitzschia kerguelensis and Thalassiosira gracilis, were selected for size variation measurements.

Donahue (1970) separated a variety Nitzschia kerguelensis var. ovalis from $N$. kerguelensis because of its thinner shells and smaller pores. The distribution of the species and its variety was determined by quantitative counts in core tops, north and south of the Antarctic Convergence. She found a striking correlation: the relative abundance of the variety north of the Antarctic Convergence increased up to $65 \%$ and south of the Antarctic Convergence it decreased. Donahue (1970) related this to nutrient supply rather than to temperature because $N$. kerguelensis is one of the most eurythermal Antarctic diatoms $\left(-1^{\circ}\right.$ to $\left.13^{\circ} \mathrm{C}\right)$. 
TABLE 1

List of Stations DSDP Leg 35, Phytoplankton Samples

\begin{tabular}{|c|c|c|c|c|c|c|c|c|c|c|c|c|c|c|c|c|c|}
\hline Station & $\begin{array}{l}\text { Temp. } \\
\text { ( } \mathrm{C} \text {. })\end{array}$ & Date & Station & $\begin{array}{c}\text { Temp. } \\
\text { (C) }\end{array}$ & Data & Station & $\begin{array}{c}\text { Temp. } \\
\text { ( } \mathrm{C} \text { ) }\end{array}$ & Date & Station & $\begin{array}{c}\text { Temp. } \\
\left.{ }^{\circ} \mathrm{C}\right)\end{array}$ & Date & Station & $\begin{array}{c}\text { Temp. } \\
\text { ( } \mathrm{C} \text {. }\end{array}$ & Date & Station & $\begin{array}{c}\text { Temp. } \\
\left({ }^{\circ} \mathrm{C}\right)\end{array}$ & Date \\
\hline$* 1^{*}$ & 18.9 & 13.2 & 52 & 19.5 & 17.2 & 104 & 15.1 & 22.2 & 154 & 7.8 & 26.2 & 204 & 5.6 & 5.3 & 254 & 1.1 & 13.3 \\
\hline 2 & 18.9 & 13.2 & 53 & 19.5 & 17.2 & $105^{*}$ & 15.1 & 22.2 & 155 & 7.8 & 26.2 & $205^{*}$ & 5.6 & 5.3 & $255^{*}$ & 1.1 & 13.3 \\
\hline 3 & 20.0 & 13.2 & 54 & 19.5 & 17.2 & 106 & 14.5 & 22.2 & 156 & 7.8 & 26.2 & 206 & 3.9 & 5.3 & 256 & 1.1 & 13.3 \\
\hline 4 & 18.3 & 13.2 & 55 & 18.9 & 17.2 & 107 & 14.5 & 22.2 & 157 & 7.2 & 27.2 & 207 & 3.9 & 5.3 & 257 & 1.1 & 13.3 \\
\hline 5 & 18.9 & 13.2 & 56 & 17.8 & 18.2 & 108 & 15.0 & 22.2 & 158 & 6.7 & 27.2 & 208 & 4.5 & 5.3 & 258 & 1.1 & 13.3 \\
\hline 6 & 18.9 & 13.2 & 57 & 17.8 & 18.2 & 109 & 15.0 & 22.2 & 159 & 6.7 & 27.2 & 209 & 3.9 & 6.3 & 259 & 1.1 & 13.3 \\
\hline 7 & 18.9 & 13.2 & 58 & 18.3 & 18.2 & 110 & 14.5 & 23.2 & $160^{*}$ & 6.7 & 27.2 & $210^{*}$ & 3.9 & 6.3 & $260^{*}$ & 1.1 & 13.3 \\
\hline 8 & 18.9 & 13.2 & 59 & 18.3 & 18.2 & 111 & 14.5 & 23.2 & 161 & 6.7 & 27.2 & 211 & 3.9 & 6.3 & 261 & 1.1 & 13.3 \\
\hline 9 & 20.0 & 14.2 & 60 & 17.2 & 18.2 & 112 & 14.5 & 23.2 & 162 & 6.7 & 27.2 & 212 & 3.9 & 6.3 & 262 & 1.1 & 13.3 \\
\hline 10 & 21.1 & 14.2 & 61 & 16.7 & 18.2 & 113 & 14.5 & 23.2 & 163 & 6.7 & 27.2 & 213 & 3.9 & 6.3 & 263 & 1.1 & 14.3 \\
\hline 11 & 20.6 & 14.2 & 62 & 17.8 & 18.2 & 114 & 14.5 & 23.2 & 164 & 6.7 & 27.2 & 214 & 3.9 & 6.3 & 264 & 1.1 & 14.3 \\
\hline 12 & 20.6 & 14.2 & 63 & 17.2 & 18.2 & $115^{*}$ & 14.5 & 23.2 & $165^{*}$ & 6.1 & 27.2 & $215^{*}$ & 3.9 & 6.3 & $265^{*}$ & 1.1 & 14.3 \\
\hline 13 & 24.5 & 14.2 & 64 & 15.6 & 18.2 & 116 & 15.0 & 23.2 & 166 & 6.1 & 28.2 & 216 & 3.9 & 6.3 & 266 & 1.1 & 14.3 \\
\hline 14 & 24.5 & 14.2 & 65 & 15.6 & 18.2 & 117 & 14.5 & 23.2 & 167 & 6.1 & 28.2 & 217 & 3.3 & 6.3 & 267 & 1.1 & 14.3 \\
\hline 15 & 24.5 & 14.2 & 66 & 15.6 & 18.2 & 118 & 14.5 & 23.2 & 168 & 6.1 & 28.2 & 218 & 3.3 & 6.3 & 268 & 1.1 & 14.3 \\
\hline 16 & 24.5 & 14.2 & 67 & 15.6 & 18.2 & 119 & 14.5 & 23.2 & 169 & 6.1 & 1.3 & 219 & 3.3 & 6.3 & 269 & 1.1 & 15.3 \\
\hline 17 & 25.0 & 14.2 & 69 & 16.1 & 19.2 & $120^{*}$ & 14.5 & 23.2 & $170^{*}$ & 6.1 & 1.3 & $220^{*}$ & 3.3 & 7.3 & $270^{*}$ & 1.1 & 15.3 \\
\hline 18 & 25.0 & 14.2 & 70 & 15.0 & 19.2 & 121 & 13.9 & 24.2 & 171 & 6.1 & 2.3 & 221 & 3.3 & 7.3 & 271 & 1.1 & 15.3 \\
\hline 19 & 24.5 & 14.2 & 71 & 14.5 & 19.2 & 122 & 13.9 & 24.2 & 172 & 6.1 & 2.3 & 222 & 3.3 & 7.3 & 272 & 1.1 & 15.3 \\
\hline 20 & 24.5 & 14.2 & 73 & 16.7 & 20.2 & 123 & 13.3 & 24.2 & 173 & 6.1 & 2.3 & 223 & 3.9 & 7.3 & 273 & 1.1 & 15.3 \\
\hline 21 & 24.5 & 15.2 & 74 & 16.7 & 20.2 & 124 & 13.3 & 24.2 & 174 & 6.1 & 2.3 & 224 & 3.9 & 7.3 & 274 & 1.7 & 15.3 \\
\hline 22 & 24.5 & 15.2 & $75^{*}$ & 17.2 & 20.2 & $125^{*}$ & 12.7 & 24.2 & $175^{*}$ & 6.1 & 2.3 & $225^{*}$ & 3.9 & 7.3 & $275^{*}$ & 1.1 & 15.3 \\
\hline 23 & 24.5 & 15.2 & 76 & 17.8 & 20.2 & 126 & 12.2 & 24.2 & 176 & 6.1 & 3.3 & 226 & 3.9 & 8.3 & 276 & 1.7 & 15.3 \\
\hline 24 & 24.5 & 15.2 & 77 & 16.7 & 20.2 & 127 & 12.8 & 24.2 & 177 & 6.1 & 3.3 & 227 & 3.3 & 8.3 & 277 & 1.7 & 15.3 \\
\hline $25^{*}$ & 24.5 & 15.2 & 78 & 16.7 & 20.2 & 128 & 12.8 & 24.2 & 178 & 6.1 & 3.3 & 228 & 3.3 & 9.3 & 278 & 1.7 & 15.3 \\
\hline 26 & 24.5 & 15.2 & 79 & 16.7 & 20.2 & 129 & 11.2 & 24.2 & 179 & 6.1 & 3.3 & 229 & 3.3 & 10.3 & 279 & 1.7 & 15.3 \\
\hline 27 & 24.5 & 15.2 & 80 & 15.0 & 20.2 & $130^{*}$ & 11.2 & 24.2 & $180^{*}$ & 6.1 & 3.3 & $230^{*}$ & 3.3 & 10.3 & $280^{*}$ & 1.7 & 15.3 \\
\hline 28 & 24.5 & 15.2 & 81 & 15.0 & 20.2 & 131 & 11.7 & 24.2 & 181 & 5.6 & 3.3 & 231 & 3.3 & 10.3 & 281 & 1.7 & 16.3 \\
\hline 30 & 22.8 & 15.2 & 82 & 15.6 & 20.2 & 132 & 10.1 & 24.2 & 182 & 5.6 & 3.3 & 232 & 3.3 & 11.3 & 282 & 1.7 & 16.3 \\
\hline 31 & 22.2 & 15.2 & 83 & 13.9 & 20.2 & 133 & 10.6 & 25.2 & 183 & 5.6 & 3.3 & 233 & 3.3 & 11.3 & 283 & 1.7 & 16.3 \\
\hline 32 & 22.2 & 15.2 & 84 & 14.4 & 20.2 & 134 & 10.0 & 25.2 & 184 & 5.6 & 3.3 & 234 & 3.3 & 11.3 & 284 & 1.7 & 16.3 \\
\hline 33 & 22.2 & 16.2 & 85 & 14.4 & 20.2 & $135^{*}$ & 10.6 & 25.2 & $185^{*}$ & 5.0 & 4.3 & $235^{*}$ & 3.9 & 11.3 & $285^{*}$ & 1.1 & 16.3 \\
\hline 34 & 22.2 & 16.2 & 86 & 15.0 & 21.2 & 136 & 10.6 & 25.2 & 186 & 5.6 & 4.3 & 236 & 3.9 & 11.3 & 286 & 1.1 & 16.3 \\
\hline 35 & 21.7 & 16.2 & 87 & 14.5 & 21.2 & 137 & 10.6 & 25.2 & 187 & 5.6 & 4.3 & 237 & 3.9 & 11.3 & 287 & 1.7 & 16.3 \\
\hline 36 & 21.7 & 16.2 & 88 & 13.9 & 21.2 & 138 & 10.6 & 25.2 & 188 & 5.6 & 4.3 & 238 & 2.8 & 11.3 & 288 & 1.7 & 16.3 \\
\hline 37 & 21.7 & 16.2 & 89 & 15.6 & 21.2 & 139 & 10.0 & 25.2 & 189 & 5.6 & 4.3 & 239 & 2.8 & 11.3 & 289 & 1.7 & 16.3 \\
\hline 38 & 22.2 & 16.2 & 90 & 15.6 & 21.2 & $140^{*}$ & 8.9 & 25.2 & $190^{*}$ & 5.6 & 4.3 & $240^{*}$ & 1.7 & 11.3 & $290^{*}$ & 1.7 & 16.3 \\
\hline 39 & 21.2 & 16.2 & 91 & 16.1 & 21.2 & 141 & 8.9 & 25.2 & 191 & 5.6 & 4.3 & 241 & 1.7 & 11.3 & 291 & 2.2 & 16.3 \\
\hline 40 & 20.6 & 16.2 & 92 & 13.9 & 21.2 & 142 & 8.3 & 25.2 & 192 & 5.6 & 4.3 & 242 & 1.1 & 12.3 & 292 & 2.8 & 17.3 \\
\hline 41 & 20.6 & 16.2 & 93 & 16.1 & 21.2 & 143 & 8.3 & 25.2 & 193 & 5.6 & 4.3 & 243 & 1.1 & 12.3 & 293 & 2.2 & 17.3 \\
\hline 42 & 20.0 & 16.2 & 94 & 16.7 & 21.2 & 144 & 8.3 & 25.2 & 194 & 5.0 & 4.3 & 244 & 1.1 & 12.3 & 294 & 2.2 & 17.3 \\
\hline 43 & 21.1 & 16.2 & 95 & 16.7 & 21.2 & $145^{*}$ & 8.3 & 26.2 & $195^{*}$ & 5.0 & 4.3 & $245^{*}$ & 1.1 & 12.3 & $295 *$ & 2.2 & 17.3 \\
\hline 44 & 21.1 & 16.2 & 96 & 16.7 & 21.2 & 146 & 8.3 & 26.2 & 196 & 5.0 & 4.3 & 246 & 1.1 & 12.3 & 296 & 2.2 & 17.3 \\
\hline 45 & 21.1 & 16.2 & 97 & 16.1 & 21.2 & 147 & 7.8 & 26.2 & 197 & 5.0 & 5.3 & 247 & 1.1 & 12.3 & 297 & 2.2 & 17.3 \\
\hline 46 & 19.5 & 17.2 & 98 & 16.1 & 22.2 & 148 & 8.3 & 26.2 & 198 & 5.0 & 5.3 & 248 & 1.7 & 12.3 & 298 & 2.2 & 17.3 \\
\hline 47 & 19.5 & 17.2 & 99 & 15.6 & 22.2 & 149 & 8.3 & 26.2 & 199 & 5.0 & 5.3 & 249 & 2.2 & 12.3 & 299 & 2.2 & 17.3 \\
\hline 48 & 19.5 & 17.2 & 100 & 16.1 & 22.2 & $150^{*}$ & 8.3 & 26.2 & 200 & 5.0 & 5.3 & 250 & 2.2 & 12.3 & $300^{*}$ & 2.2 & 17.3 \\
\hline 49 & 19.5 & 17.2 & $101^{*}$ & 16.1 & 22.2 & 151 & 8.3 & 26.2 & $201 *$ & 4.5 & 5.3 & 251 & 1.1 & 12.3 & 301 & 2.2 & 18.3 \\
\hline $50^{*}$ & 20.0 & 17.2 & 102 & 16.1 & 22.2 & 152 & 7.8 & 26.2 & 202 & 5.0 & 5.3 & $252 *$ & 1.1 & 12.3 & 302 & 2.2 & 18.3 \\
\hline \multirow[t]{5}{*}{51} & 20.0 & 17.2 & 103 & 15.0 & 22.2 & 153 & 7.8 & 26.2 & 203 & 5.0 & 5.3 & 253 & 1.1 & 12.3 & 303 & 2.2 & 18.3 \\
\hline & & & & & & & & & & & & & & & 304 & 2.2 & 18.3 \\
\hline & & & & & & & & & & & & & & & $305^{*}$ & 2.2 & 18.3 \\
\hline & & & & & & & & & & & & & & & 306 & 2.2 & 18.3 \\
\hline & & & & & & & & & & & & & & & $307^{*}$ & 2.2 & 18.3 \\
\hline
\end{tabular}

Note: Asterisk denotes stations treated in this paper.

It was impossible, in the phytoplankton material on hand, to separate the variety from the species, but by using the ratio of the length to the width of the species multiplied by the width of 5 costae, and using counts of 100-200 individuals in 10 widely separated plankton samples, a factor, $F$, involving these parameters was calculated:

$$
\left.F=\frac{\text { length }}{\text { width }} \times \text { width of } 5 \text { costae (in } \mu \mathrm{m}\right)
$$

and the mean value

$$
F=\frac{\sum_{1}^{n} F}{n}
$$

was plotted versus temperature. Standard deviation was calculated using the formula:

$$
\mathrm{SD}=\sqrt{\frac{F^{2}-\frac{(F)^{2}}{n}}{n-1}}
$$

Figure 8, which graphs the results of the study, demonstrates a continuous increase in $F$ values from north to south. Smaller individuals of Nitzschia kerguelensis with more costae in $5 \mu \mathrm{m}$ occur north of the Antarctic Convergence with a continuous increase of individuals with larger shells and with fewer costae in $5 \mu \mathrm{m}$ southwards. No distinct deviation from this tendency is observed across the Antarctic Convergence 


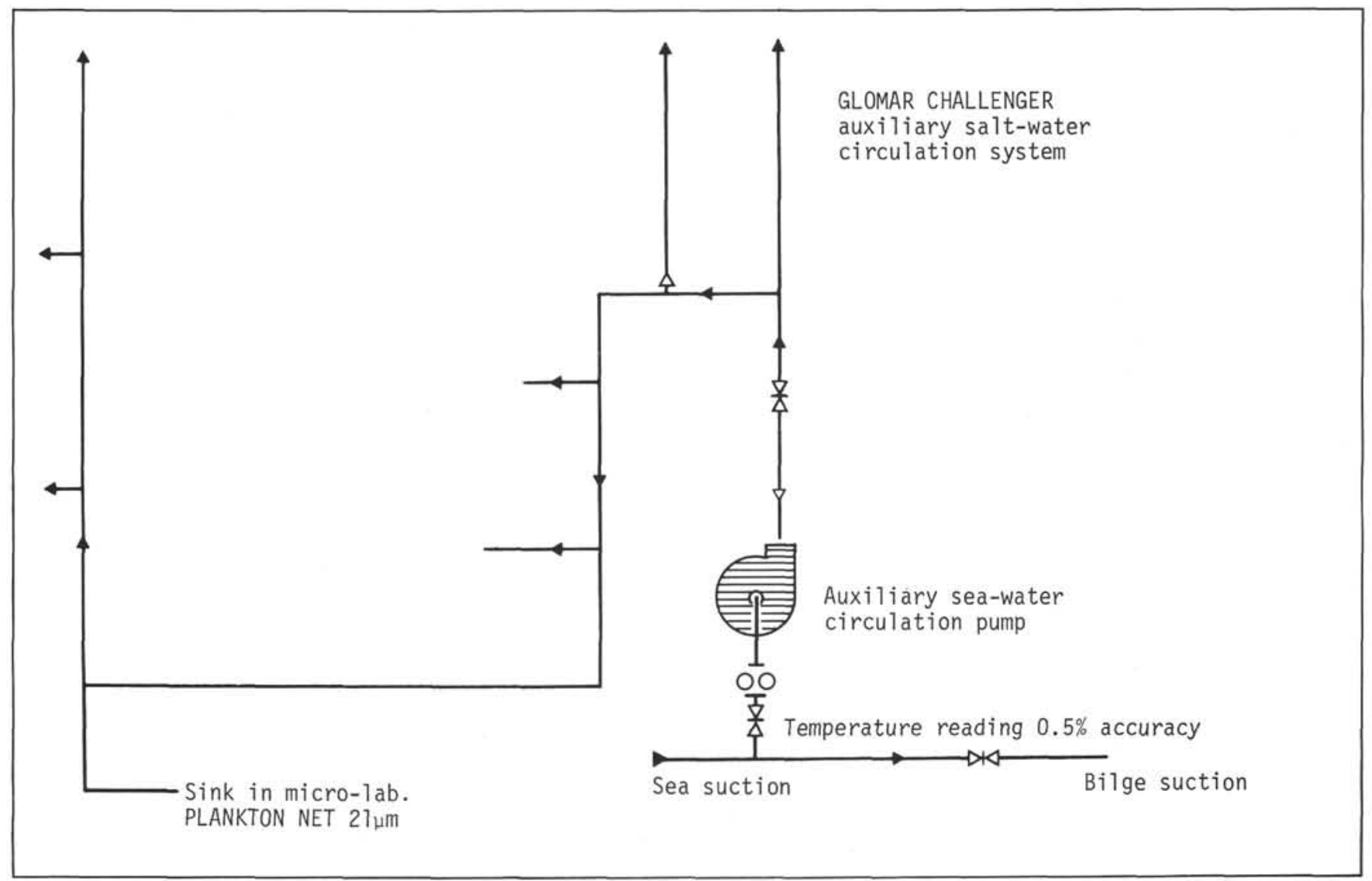

Figure 4. Schematic description of the salt-water system from the seawater inlet (4m below surface on the ship's hull) to outlet in microlaboratory.

$\left(64^{\circ} 30^{\prime}\right.$ to $63^{\circ} 30^{\prime} \mathrm{S}$ latitude) or in approaching and reaching the Antarctic Divergence $\left(68^{\circ}\right.$ to $70^{\circ} \mathrm{S}$ latitude). Temperature decreases continuously over this section (from $53^{\circ}$ to $69^{\circ} \mathrm{S}$ latitude) and thus there appears to be a direct correlation of $F$ with temperature rather than correlation with nutrients because the highest nutrient values occur over the Antarctic Convergence and the Antarctic Divergence.

Nitzschia kerguelensis has a wide variation in valve length, width, and width of 5 costae; it was impossible to distinguish among Nitzschia kerguelensis, Nitzschia kerguelensis var. ovalis, and Nitzschia lanceolata as has been proposed by Donahue (1970). A small elliptical form with (Plate 2, Figures 19-30) nearly parallel margins was found south of the Antarctic Convergence in small percentages $(<1 \%$ of the total Nitzschia kerguelensis fraction); it is interpreted to represent a race of $N$. kerguelensis and was not separated taxonomically from the species.

Valve shape and ornamentation of the species thus may serve in paleoecological investigations as indicators of past temperature fluctuations in, or mass movements of, Antarctic waters. N. kerguelensis in core top sediments retrieved from DSDP Leg 35 served well as an indicator of the northward movement of the Antarctic Bottom Water transporting enroute endemic Antarctic floral elements into subtropical/tropical areas.
Thalassiosira gracilis is a characteristic diatom species in Antarctic waters, with a distribution limited to south of $58^{\circ} \mathrm{S}$ latitude. Frustules are strongly silicified and frequently found in Antarctic sediments of Quaternary and Pliocene age (McCollum, 1975; Donahue, 1970). Some taxonomic uncertainties exist in distinguishing the species from Cosmiodiscus insignis; supposedly they are the same species but with different diameters.

One hundred individuals of Thalassiosira gracilis were measured in each plankton sample and the mean diameter and standard deviation were calculated (Figure 9). Mean diameter reveals no direct correlation with surface water temperatures being of comparable value north of $65^{\circ} \mathrm{S}$ latitude and south of $67^{\circ} \mathrm{S}$ latitude. Maximum diameters were found to occur between $65^{\circ}$ and $67^{\circ} \mathrm{S}$ latitude. This peak lies between the Antarctic Divergence and Convergence zones and may serve as another index in reconstruction of past Antarctic circulation patterns. Further, there may be some relationship between the maximum diameter mean and the lower diversity values within this zone (Figures 6 and 7), as a function of it being less competitive.

\section{Distribution of Eucampia balaustium}

Eucampia balaustium has not been recorded beyond the Southern Ocean, but its distribution therein is controversial. Hendey (1937), Hustedt (1958), and Hasle 
(1969) regard the species to be widely distributed throughout the Southern Ocean, whereas Hart (1934), Manguin (1960), Fukase (1962), Cassie (1963), Fukase and El-Sayed (1965), and Kozlova (1964) did not observe the species north of the Antarctic Convergence. Abbott (1972) found it to be abundant throughout the Subantarctic in surface sediment layers of the western South Pacific Ocean, with a relative abundance of up to $5 \%$ of the total diatom assemblage. Hasle found the largest populations, from 100,000 to 500,000 cells per liter, in Brategg section I, north of the Antarctic Convergence. Eucampia balaustium occurs as traces, in the present material with notable percentages only in Samples 245, 195, 175, 170, and 165. All of these samples, except 245 and 195, are situated north of the Antarctic Convergence. The species will be enriched in sediment assemblages because of its heavy silicified frustules, which resist dissolution during descend and burial. Gombos (1974) reported Eucampia balaustium together with Charcotia actinochilus as being associated with ice conditions, "these two species are very abundant during periods of cooling and extended ice front, but are absent during periods of relative warming and receded ice front." Abbott (1972) calculated climatic changes using a ratio of the number of Subantarctic Coscinodiscus lentiginosus to the combined number of the Antarctic species Eucampia balaustium and Charcotia actinochilus; he found good correlation to climatic curves based on other parameters, i.e., foraminifera, radiolarians, sand, and $\mathrm{CaCO}_{3}$ content. The assumption that Eucampia balaustium is an ice-dwelling species cannot be drawn from our phytoplankton assemblage, nor do sediment data available in the literature support the assumption. Useful are the data of Abbott (1972), who found higher percentages north of, and lower percentages south of $55^{\circ} \mathrm{S}$. The data represented by Figure 10 shows Eucampia balaustium to have its greatest abundance in the open ocean from $63^{\circ}$ to $56^{\circ} \mathrm{S}$ latitude, but in light of Hart's (1934) report that the species has been found abundantly in samples taken near South Georgia $\left(55-54^{\circ} \mathrm{S}\right.$ latitude) and in samples taken at the eastern end of the Bainsfield Strait and at the Palmer Archipelago, no unequivocal conclusion can be drawn on the latitudinal distribution of the species.

\section{ACKNOWLEDGMENTS}

The investigation was supported by the Deutsche Forschungsgemeinschaft and is part of one of the author's (Schrader) participation in the upwelling program (Auftriebsphänomene im Meer) of the German National Science Foundation and the CLIMAP program of the U.S. National Science Foundation. The authors are grateful to fellow colleagues, Fred Rögl and Dennis Graham without whose help continuous sampling during day and night would have been impossible. The authors further acknowledge their indebtedness to the crew of the Glomar Challenger, especially the engine room crew who recorded water temperatures. Frau Schmidtmann and Frau Mielck helped in typing and proof reading.

\section{SYSTEMATIC SECTION, FLORAL REFERENCES}

In the following pages genera are arranged alphabetically as are species within each genus. Some species, the taxonomic positions of which are unclear, and which need ultimate revision, are discussed in groups. Table 3 presents a classification used in modern taxonomy, which will facilitate the reader with a general idea of the arrangement of the genera, families, orders, etc., in a "natural" system (from Simonsen, 1974). Species and variety descriptions follow those of Hustedt (1930-1959), Hasle (1965), Simonsen (1974), and others; differences from descriptions of those authors are noted under Remarks. For the more important species, a length/width correlation is included to demonstrate extremes and maximum clustering. As many species and variations within a species as possible have been illustrated (Plates 1-14).

\section{Genus ACTINOCYCLUS Ehrenberg (1837)}

\section{Actinocyclus curvatulus Janisch in Schmidt (1878)} (Plate 6, Figures 1, 2)

Description: Hustedt (1930), p. 538, fig. 307.

Remarks: Valves flat, discoid, Valve diameter: 32-67 $\mu \mathrm{m}$. Number of loculi: $9-15$ in $10 \mu \mathrm{m}$. The loculi are arranged irregularly in the small central area forming a cluster which is surrounded by a hyaline ring. The loculi are grouped in fascicles, the first row of each fascicle running from the valve center to the margin with the shorter rows parallel to the first row; rows somewhat curved. The loculi near the margin are distinctly smaller (12-17 in $10 \mu \mathrm{m})$. Marginal labiate processes lie in the continuation of the longest row. The submarginal pseudonodulus is, in some specimens, discernible only with difficulty.

This species is bound to colder water masses in both hemispheres (Hustedt, 1930), but it seems to be more frequent in the Antarctic and Subantarctic region.

Actinocyclus curvatulus was found in DSDP Leg 35 samples only in Subantarctic waters, which is in good agreement with Van Heurck (1909), Mann (1937), Hustedt (1958), Hargraves (1968), and Abbott (1972). Hasle (1960), however, reported the species, with a structure more similar to Thalassiosira eccentrica, from the tropical Pacific Ocean.

\section{Actinocyclus ehrenbergii Ralfs (1861)}

(Plate 5, Figures 1, 2, 10)

Description: Hustedt (1930), p. 525-528, fig. 298.

Remarks: The observed specimens are $48-103 \mu \mathrm{m}$ in diameter. Number of loculi: 9-10 in $10 \mu \mathrm{m}$, and is very constant on one valve. In the abrupt beginning submarginal zone, 14-16 loculi in $10 \mu \mathrm{m}$ occur. Marginal spines in continuation of the interfasciculate rows are easily discernible and vary in number with the fascicles from 11 to 17 . The pseudonodulus lies at the upper border of the submarginal zone, the width of which is always smaller than $1 / 10$ of the valve diameter. The central area varies in diameter from $2-5 \mu \mathrm{m}$. Either the central area is hyaline with just one eccentric loculus (Plate 5, Figure 10), or is filled with irregularly arranged loculi, this central areolae-bulk being separated from the other areolae by a hyaline ring (Plate 5, Figure 1, 2).

Actinocyclus ehrenbergii was found only at the northern stations of DSDP Leg 35, from $13^{\circ} \mathrm{S}$ to $41^{\circ} \mathrm{S}$ latitude. Hustedt (1958, p. 130) reports single specimens of the species in temperate regions in the South Atlantic as far south as $59^{\circ} \mathrm{S}$ latitude.

\section{Actinocyclus ehrenbergii var. tenella (Bréb.) Hustedt (1930)}

$$
\text { (Plate 5, Figures 3, 4) }
$$

Description: Hustedt (1930), p. 530, fig. 302.

Remarks: Two individuals of this variety were found in the examined materials, at station $1\left(13^{\circ} \mathrm{S}\right.$ latitude $)$ and station $230\left(63.5^{\circ} \mathrm{S}\right.$ latitude). The specimens are $26.5 \mu \mathrm{m}$ and $32 \mu \mathrm{m}$ in diameter. The number of loculi in $10 \mu \mathrm{m}$ is $9-11$ on the flat part of the valve and 15 16 in the submarginal zone.

\section{Genus ACTINOPTYCHUS Ehrenberg (1839)}

\section{Actinoptychus aff. splendens (Shadb.) Ralfs in Pritchard (1861) (No illustration)}

Description: Hustedt (1930), p. 478-479, fig. 265.

Remarks: Only one specimen of this species was found at station $101,41.7^{\circ} \mathrm{S}$ latitude. The species is common in warm and temperate waters (Hustedt, 1930). The specimen is $30 \mu$ in valve diameter, and has 15 pores in $10 \mu \mathrm{m}$, the number of which increases slightly towards the margin. The central subcircular hyaline area is $5 \mu \mathrm{m}$ in diameter. The number of sections is 10 , with raised sections possessing a 
TABLE 2A

Percent Abundance of Diatom Species Found Within Each Sample

\begin{tabular}{|c|c|c|c|c|c|c|c|c|c|c|c|c|c|c|c|c|c|c|c|c|c|c|c|}
\hline & 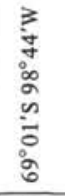 & $\begin{array}{l}3 \\
\hat{\sigma} \\
\infty \\
o \\
\text { in } \\
\text { in } \\
0 \\
0\end{array}$ & $\begin{array}{l}3 \\
\text { ì } \\
\text { o } \\
\text { o } \\
\text { is } \\
\text { iे } \\
\vdots \\
0\end{array}$ & 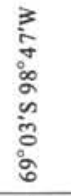 & 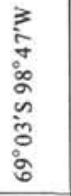 & $\begin{array}{l}3 \\
i n \\
i \\
i \\
i \\
\bar{\sigma} \\
\infty\end{array}$ & $\begin{array}{l}3 \\
i \\
0 \\
i \\
0 \\
i \\
0 \\
0 \\
0\end{array}$ & $\begin{array}{l}3 \\
i \\
i n \\
0 \\
\infty \\
i n \\
i \\
i \\
\vdots \\
0\end{array}$ & $\begin{array}{l}3 \\
\frac{1}{2} \\
\delta \\
0 \\
\tilde{\omega} \\
\vdots \\
\vdots\end{array}$ & 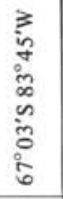 & $\begin{array}{l}3 \\
0 \\
0 \\
\infty \\
0 \\
0 \\
0 \\
0 \\
0\end{array}$ & 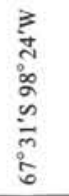 & $\begin{array}{l}3 \\
\text { in } \\
\text { o } \\
\text { in } \\
\text { in } \\
\text { iे } \\
\text { i. }\end{array}$ & $\begin{array}{l}3 \\
0 \\
\infty \\
\infty \\
\infty \\
\infty \\
0 \\
0 \\
0\end{array}$ & 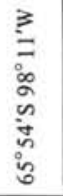 & $\begin{array}{l}3 \\
0 \\
\infty \\
\infty \\
0 \\
0 \\
0 \\
0 \\
0\end{array}$ & $\begin{array}{l}3 \\
0 \\
\vdots \\
\vdots \\
\text { in } \\
\text { a } \\
0 \\
0\end{array}$ & 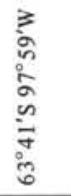 & 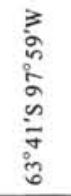 & $\begin{array}{l}3 \\
0 \\
\vdots \\
\vdots \\
0 \\
\vdots \\
0 \\
0 \\
0\end{array}$ & 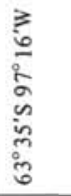 & 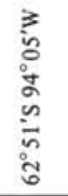 & 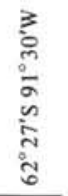 \\
\hline Station No. & $\tilde{\varkappa}$ & $\tilde{\text { }}$ & : & $\tilde{\sim}$ & $\stackrel{2}{\sim}$ & $\cong$ & $\underset{\sim}{\sim}$ & $\stackrel{\sim}{\infty}$ & Дิ & ฉั & \&్ల & $\stackrel{2}{2}$ & 总 & 㝏 & $\stackrel{P}{d}$ & $\stackrel{\sim}{\sim}$ & స̊ำ & $\approx$ & สิ & $\frac{n}{2}$ & $\stackrel{2}{2}$ & $\cong$ & $\bar{\AA}$ \\
\hline Temperature $\left({ }^{\circ} \mathrm{C}\right)$ & 1.1 & 1.1 & 1.1 & 1.1 & 1.1 & 1.1 & 1.7 & 1.1 & 1.7 & 2.2 & 2.2 & 1.1 & 2.2 & 2.2 & 1.7 & 3.9 & 3.3 & 3.9 & 3.3 & 3.9 & 3.9 & 5.6 & 4.5 \\
\hline $\begin{array}{l}\text { Actinocyclus curvatulus } \\
\text { A. ehrenbergii } \\
\text { A. ehrenbergii vartenella } \\
\text { Actinoptychus splendens } \\
\text { Amphora aff. biarcuata }\end{array}$ & . & & & & & & & & & & & & & & & & $1:$ & & & & & & \\
\hline $\begin{array}{l}\text { Amphora aff. sp. } \\
\text { Asteromphalus heptactis }\end{array}$ & & & & & & & & & & & & & & & & & & & & & & & \\
\hline A. hookeri & & & 0.2 & & & & & & 0.3 & & & 0.4 & 0.3 & 0.3 & & & 0.2 & & 0.2 & & & & \\
\hline $\begin{array}{l}\text { A. hyalinus } \\
\text { A. parvulus }\end{array}$ & 4 & $\begin{array}{l}1.5 \\
0.3\end{array}$ & $\begin{array}{l}1.6 \\
1.2\end{array}$ & $\begin{array}{l}1.2 \\
0.6\end{array}$ & 2 & $\begin{array}{l}2.4 \\
0.9\end{array}$ & $\begin{array}{l}4.4 \\
0.9\end{array}$ & $\begin{array}{l}0.6 \\
0.3\end{array}$ & $\begin{array}{l}0.3 \\
2.2\end{array}$ & $\begin{array}{l}7.3 \\
0.3\end{array}$ & $\begin{array}{l}1.3 \\
0.2\end{array}$ & $\begin{array}{l}0.9 \\
1.1\end{array}$ & $\begin{array}{l}1.2 \\
0.3\end{array}$ & $\begin{array}{l}9 \\
1.1\end{array}$ & 2.8 & $\begin{array}{l}2.3 \\
0.3\end{array}$ & & 0.2 & 0.2 & & & 1.2 & 0.4 \\
\hline Bacteriastrum hyalinum & & & & & & & & & & & & & & & & & & & & & & & \\
\hline $\begin{array}{l}\text { Biddulphia longicruris } \\
\text { B. sp. } \\
\text { Chaetoceros atlanticum }\end{array}$ & & & & & & & & & & & & & & & & 0.3 & & & & & & & 0.4 \\
\hline $\begin{array}{l}\text { f. bulbosum } \\
\text { C. sp. }\end{array}$ & $\begin{array}{r}3.6 \\
10.5\end{array}$ & 18.2 & 14.2 & 10.2 & 4.7 & $\begin{array}{l}1.7 \\
2.8\end{array}$ & 5.6 & 4.8 & $\begin{array}{l}0.3 \\
2.2\end{array}$ & 4.2 & 2.2 & 0.7 & $\begin{array}{l}0.3 \\
3\end{array}$ & 7.9 & 2 & 0.3 & & & & & 0.3 & & \\
\hline $\begin{array}{l}\text { Charcotia actinochilus } \\
\text { Chorethron criophilum } \\
\text { Cocconeis aff. placentula } \\
\text { C. costata var. antarctica } \\
\text { Coscinodiscus asteromphalus }\end{array}$ & $\begin{array}{l}0.2 \\
0.2\end{array}$ & 0.3 & 0.9 & 0.6 & 1 & & & 28.3 & 0.6 & & 1 & $\begin{array}{l}0.2 \\
0.2\end{array}$ & 0.3 & 0.3 & & & & & & & & & 0.4 \\
\hline $\begin{array}{l}\text { C. bullatus } \\
\text { C. crenulatus }\end{array}$ & & 0.6 & 0.5 & 0.3 & 0.6 & & 0.3 & 0.3 & & & 0.3 & 0.4 & & 2.5 & & & 1.1 & 1.9 & 1.1 & 1.3 & 0.5 & 0.5 & $\overline{0.4}$ \\
\hline $\begin{array}{l}\text { C. curvatulus } \\
\text { C. kutzingi }\end{array}$ & 0.2 & 0.3 & & & & & & & 0.3 & & & & & 0.3 & 2 & 1.5 & 5.3 & 5.1 & 3.7 & 1.9 & 1 & 1.7 & 5.9 \\
\hline C. lentiginosus & 1.6 & 0.3 & 0.2 & 0.6 & 0.3 & 0.7 & 0.3 & 0.3 & & 0.3 & 0.3 & 0.9 & & 1.4 & 2.8 & 2.3 & 2.4 & 2.6 & 3.3 & 1.3 & 1 & & 0.4 \\
\hline $\begin{array}{l}\text { C. lineatus } \\
\text { C. perforatus } \\
\text { C. radiatus } \\
\text { C. stellaris var. symbolophorus } \\
\text { C. tabularis }\end{array}$ & 0.5 & & & & & 0.2 & 0.3 & & & 0.6 & 0.2 & 0.2 & 0.3 & 0.8 & 2.8 & 0.3 & & 0.2 & 0.2 & 0.3 & & & 0.4 \\
\hline $\begin{array}{l}\text { C. tabularis var. egregius } \\
\text { Cyclotella stylorum } \\
\text { Dactyliosolen antarcticus } \\
\text { Eucampia balaustium } \\
\text { Hemidiscus cuneiformis }\end{array}$ & & & & & & & & & 0.3 & & & 0.2 & & & 0.3 & & & & & & 0.3 & 0.7 & \\
\hline $\begin{array}{l}\text { Lithodesmium undulatum } \\
\text { Navicula directa } \\
\text { N. trompii }\end{array}$ & 0.2 & & & & 0.3 & & & & 0.3 & & & & & 0.3 & & 0.5 & 2.7 & 1.4 & & & & 0.7 & \\
\hline $\begin{array}{l}\text { Nitzschia angulata } \\
\text { N. bicapitata }\end{array}$ & $\begin{array}{l}2.5 \\
0.5\end{array}$ & 6.4 & 4.2 & 11.7 & 7.8 & 1.3 & 5.6 & 2.4 & 5.3 & 9.7 & 3.3 & 6.1 & 5.5 & 8.4 & 0.6 & 4.8 & 4.9 & 0.5 & 1.1 & 0.5 & 1.6 & $\begin{array}{l}1.9 \\
0.2\end{array}$ & 6.3 \\
\hline N. curta & 0.4 & 5.2 & 4.2 & 2.5 & 3.3 & 0.4 & 2.7 & 5.8 & 3.4 & 1.2 & 1.9 & 8.3 & 3.6 & 1.7 & 0.3 & 0.5 & 0.2 & 0.2 & & & & & \\
\hline $\begin{array}{l}\text { N. cylindrus } \\
\text { N. fraudulenta } \\
\text { N. grunowii }\end{array}$ & 2.2 & 21.1 & 29.4 & 20.6 & 30.8 & 2.6 & 11.1 & 24.1 & 18.3 & $\begin{array}{l}2.4 \\
0.3\end{array}$ & 2.2 & 31.1 & 2.7 & $\begin{array}{l}3.7 \\
0.3\end{array}$ & 0.8 & $\begin{array}{l}5.0 \\
0.3\end{array}$ & 4.7 & 0.2 & 2.0 & & 0.3 & & \\
\hline N. heimii & & & & & & & & & & & & & & & & 0.5 & & 0.7 & 0.7 & 0.3 & 0.5 & 14.4 & 5.4 \\
\hline $\begin{array}{l}\text { N. kerguelensis } \\
\text { N. kolaczeckii }\end{array}$ & 57.2 & 22.5 & 21.7 & 31.7 & 26.4 & 62.2 & 40.9 & 24.9 & 29.6 & 5.0 & 76.4 & 21.7 & 77.3 & 40.2 & 72.6 & 54.8 & 66.7 & 74.1 & 69.6 & 75.4 & 66.6 & 18.8 & 43.5 \\
\hline
\end{tabular}




\begin{tabular}{|c|c|c|c|c|c|c|c|c|c|c|c|c|c|c|c|c|c|c|c|c|c|c|c|}
\hline $\begin{array}{l}\text { N. lineola } \\
\text { N. obliquecostata } \\
\text { N. peragalli } \\
\text { N. pseudonana } \\
\text { N. pungens }\end{array}$ & 0.2 & $\begin{array}{l}0.3 \\
4.1\end{array}$ & $\begin{array}{l}0.2 \\
0.2\end{array}$ & $\begin{array}{l}0.6 \\
0.6\end{array}$ & $\begin{array}{l}0.7 \\
0.3\end{array}$ & $\begin{array}{l}1.1 \\
1.1\end{array}$ & $\begin{array}{l}0.9 \\
0.6\end{array}$ & 2.6 & 0.3 & $\begin{array}{l}0.9 \\
0.9\end{array}$ & 0.2 & $\begin{array}{l}4.4 \\
0.2 \\
4.8\end{array}$ & & 0.6 & & 2 & 0.2 & & & 0.3 & & 1.9 & 0.7 \\
\hline N. ritscheri & 0.2 & & & 0.3 & & & & & & 0.3 & 0.9 & 0.4 & 0.3 & 0.3 & 0.3 & 0.8 & 0.2 & 0.5 & & 0.5 & & 0.7 & 0.4 \\
\hline $\begin{array}{l}\text { N. separanda } \\
\text { N. seriata } \\
\text { N. sicula var, bictuneata } \\
\text { N. sicula var, rostrata } \\
\end{array}$ & 0.2 & 0.9 & 3.5 & 0.9 & 3 & 4.1 & 3.2 & 1 & 5.2 & 11.5 & 1.7 & 2.2 & 1.2 & 11.2 & 1.4 & 0.8 & 0.7 & & 0.2 & 0.5 & & 0.2 & 1.1 \\
\hline $\begin{array}{l}\text { N. turgidula } \\
\text { N. turgiduloides } \\
\text { N. vanheurckii } \\
\text { Pleurosigma directum } \\
\text { Pseudoeunotia doliolus }\end{array}$ & & & & & & & & & & & & & & & & 0.3 & & & & & $\begin{array}{l}0.3 \\
0.3\end{array}$ & 0.2 & $\begin{array}{l}0.4 \\
0.4 \\
\end{array}$ \\
\hline $\begin{array}{l}\text { Rhizosolenia alata } \\
\text { R. alata forma inermis } \\
R \text {. hergonii } \\
R \text {. hebetata forma bidens } \\
\text { R. hebetata forma hiemalis } \\
\end{array}$ & 0.5 & & & 0.3 & & & & 0.3 & & & 0.2 & 0.4 & & & & 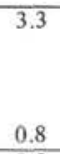 & 1.1 & 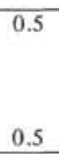 & 2.4 & 1.1 & 11.8 & 43.8 & 11.8 \\
\hline $\begin{array}{l}\text { R. hebetata forma semispina } \\
\text { R. styliformis } \\
\text { Roperia tesselata }\end{array}$ & & & & & & & & & & & & & & & 0.3 & $\begin{array}{l}0.5 \\
2.8\end{array}$ & 0.4 & 0.9 & 0.9 & 0.8 & 3.9 & $\begin{array}{l}1 \\
5.1\end{array}$ & $\begin{array}{l}1.1 \\
3\end{array}$ \\
\hline $\begin{array}{l}\text { Schimperiella antarctica } \\
\text { Thalassionema nitzschioides }\end{array}$ & 0.2 & 0.6 & 0.2 & 0.3 & 0.3 & 0.2 & 0.6 & 0.3 & 0.3 & 0.3 & 0.3 & 0.2 & 0.3 & 0.6 & 1.7 & 4 & 2 & 3.9 & 2.4 & 3.5 & 2.1 & 1 & 4.8 \\
\hline $\begin{array}{l}\text { T. nitzschioides var. parva } \\
\text { Thalassiosira decipiens } \\
\text { T. delicatula }\end{array}$ & & 0.9 & & & 0.7 & & 0.3 & & & & & & & & & & 0.4 & & & & & $\begin{array}{l}1 \\
0.5\end{array}$ & \\
\hline $\begin{array}{l}\text { T. eccentrica } \\
\text { T. eccentrica var. jousei }\end{array}$ & & & & & & 0.4 & & & 0.3 & & & 0.9 & & 0.3 & 0.8 & 0.5 & $\begin{array}{l}0.4 \\
0.4\end{array}$ & $\begin{array}{l}0.9 \\
0.2\end{array}$ & 4.3 & 3.2 & 4.7 & & $\begin{array}{l}0.7 \\
0.4\end{array}$ \\
\hline $\begin{array}{l}\text { T. gracilis } \\
\text { T. gravida } \\
\text { T. lineata } \\
\text { T. oestrupii } \\
\text { T. porosenata } \\
\end{array}$ & 6.5 & 16.2 & 12.6 & 16.6 & 15.9 & 16.8 & 21.6 & 3.3 & 29.6 & 8.5 & 6 & 13.2 & 1.8 & 6.2 & $\begin{array}{l}7.6 \\
0.6\end{array}$ & 6.3 & 3.6 & 3.7 & 2.4 & $\begin{array}{l}2.1 \\
0.5\end{array}$ & $\begin{array}{l}1.3 \\
0.8\end{array}$ & $\begin{array}{l}1.9 \\
0.2\end{array}$ & 0.4 \\
\hline $\begin{array}{l}\text { T. symmetrica } \\
\text { T. tumida } \\
T \text {. sp. a } \\
T \text {, sp. b } \\
T \text {. sp. c } \\
\end{array}$ & 0.2 & 0.6 & & 0.6 & 0.7 & 0.2 & & & & & 0.3 & & & 0.6 & 0.6 & 0.3 & 0.2 & 0.2 & 1.1 & $\begin{array}{l}0.3 \\
1.6 \\
\\
0.5 \\
0.3 \\
\end{array}$ & $\begin{array}{l}0.3 \\
0.8 \\
0.8\end{array}$ & 0.2 & $\begin{array}{l}0.4 \\
1.5\end{array}$ \\
\hline $\begin{array}{l}T . \text { sp.d } \\
T \text {. sp. e } \\
T \text {. sp. f }\end{array}$ & & & & & & & & & 0.9 & & 0.2 & & & & & 2 & & 0.7 & 0.7 & & 0.8 & & 1.9 \\
\hline $\begin{array}{l}T \text {. sp. g } \\
\text { Thalassiothrix longissima }\end{array}$ & 0.5 & & & & & 0.4 & & 0.3 & & 0.6 & 1 & 0.2 & 0.9 & 2 & 0.6 & 1.8 & 1.6 & 0.2 & 0.4 & 1.3 & 0.5 & 1 & 0.7 \\
\hline $\begin{array}{l}\text { Trachyneis aspera } \\
\text { Tropidoneis }\end{array}$ & 0.2 & & & & & & & & & & & 0.2 & & 0.3 & & 0.3 & & & 0.2 & 0.3 & & & \\
\hline
\end{tabular}


TABLE 2B

Percent Abundance of Diatom Species Found Within Each Sample

\begin{tabular}{|c|c|c|c|c|c|c|c|c|c|c|c|c|c|c|c|c|c|c|c|c|c|c|}
\hline & $\begin{array}{l}3 \\
0 \\
\infty \\
\infty \\
0 \\
0 \\
0 \\
0 \\
\bar{b}\end{array}$ & 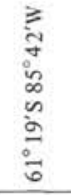 & 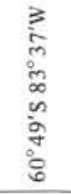 & 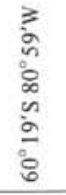 & 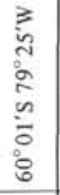 & $\begin{array}{l}3 \\
\text { iे } \\
\text { oे } \\
\text { ¿े } \\
\tilde{0} \\
0 \\
0 \\
0\end{array}$ & 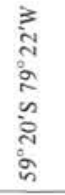 & 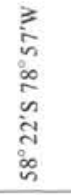 & $\begin{array}{l}\geqslant \\
\infty \\
o \\
\infty \\
\tilde{\omega} \\
\ddot{w} \\
i \\
i n\end{array}$ & 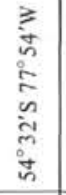 & 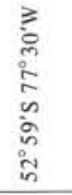 & $\begin{array}{l}\geqslant \\
0 \\
0 \\
\text { ह } \\
\text { in } \\
\text { in } \\
\text { on } \\
\text { in }\end{array}$ & 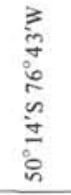 & $\begin{array}{l}\geqslant \\
0 \\
i \\
0 \\
i n \\
i \\
i 0 \\
\infty \\
\infty \\
0\end{array}$ & $\begin{array}{l}3 \\
\text { ì } \\
\text { jo } \\
\tilde{\omega} \\
\ddot{c} \\
\vdots \\
\vdots\end{array}$ & $\begin{array}{l}3 \\
\text { aे } \\
\text { in } \\
\text { in } \\
\text { wे } \\
\text { in } \\
\text { f }\end{array}$ & 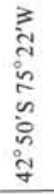 & 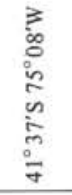 & 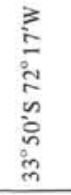 & 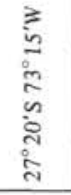 & 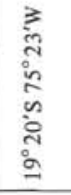 & 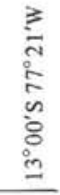 \\
\hline & $\cong$ & 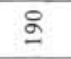 & $\cong$ & $\cong$ & $\stackrel{n}{\cong}$ & 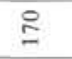 & 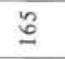 & $\stackrel{8}{0}$ & 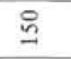 & $\stackrel{n}{z}$ & I & $\cong$ & 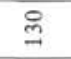 & $\cong$ & ิ & $\cong$ & $\stackrel{\circ}{\varrho}$ & $\vec{\Xi}$ & $\approx$ & 8 & $\approx$ & - \\
\hline & 5 & 5.6 & 5 & 6.1 & 6.1 & 6.1 & 6.1 & 6.7 & 8.3 & 8.3 & 8.9 & 10.6 & 11.2 & 12.8 & 14.5 & 14.5 & 15 & 15.6 & 17.2 & 20 & 24.5 & 18.9 \\
\hline $\begin{array}{l}\text { Actinocyclus curvatulus } \\
\text { A. ehrenbergii } \\
\text { A. ehrenbergii var, tenella } \\
\text { Actinoptychus splendens } \\
\text { Amphora aff. biarcuata }\end{array}$ & & & & & & & & & & & & 0.7 & 2.7 & & + & & + & $\begin{array}{l}0.3 \\
1.4 \\
0.3\end{array}$ & $\begin{array}{l}+ \\
+\end{array}$ & & $\begin{array}{l}+ \\
+\end{array}$ & $\begin{array}{l}0.3 \\
0.3 \\
0.6 \\
8.3\end{array}$ \\
\hline $\begin{array}{l}\text { A. sp. } \\
\text { Asteromphalus heptactis } \\
\text { A. hookeri } \\
\text { A. hyalinus } \\
\text { A. parvulus }\end{array}$ & & 0.3 & & 0.6 & 0.4 & 0.9 & 0.6 & $\begin{array}{l}0.4 \\
0.1 \\
0.1\end{array}$ & 1.3 & $\begin{array}{l}0.4 \\
0.4\end{array}$ & $\begin{array}{l}1.3 \\
0.2 \\
0.8\end{array}$ & $\begin{array}{l}0.4 \\
0.4 \\
\end{array}$ & & & & + & + & & & & & 0.3 \\
\hline $\begin{array}{l}\text { Bacteriastrum hyalinum } \\
\text { Biddulphia longicruris } \\
\text { B. sp. } \\
\text { Chaetoceros atlanticum } \\
\text { f. bulbosum }\end{array}$ & 0.2 & & & & & & & & & & & & & & + & & + & -1 & + & & & $\begin{array}{l}0.6 \\
6.8\end{array}$ \\
\hline C. sp. & 9.3 & 12.3 & 13.1 & 1.5 & 4.8 & 4.8 & 0.6 & 1 & & 0.6 & 0.2 & 1.8 & 1.5 & & t+ & + & + & 0.3 & + & 16.8 & + & 10.3 \\
\hline $\begin{array}{l}\text { Charcotia actinochilus } \\
\text { Chorethron criophilum } \\
\text { Cocconeis aff. placentula } \\
\text { C. costata var. antarctica } \\
\text { Coscinodiscus asteromphalus }\end{array}$ & 1.2 & & 1.6 & 0.3 & & 0.3 & & 0.2 & & & & & & & $\begin{array}{l}+ \\
+\end{array}$ & + & + & & + & & & 17.5 \\
\hline \begin{tabular}{l|} 
C. bullatus \\
C. crenulatus \\
C. curvatulus \\
C. kutzingi \\
C. lentiginosus
\end{tabular} & $\begin{array}{l}0.4 \\
0.8 \\
0.2\end{array}$ & 3.7 & 0.6 & 2.1 & 0.6 & $\begin{array}{l}2.3 \\
0.6\end{array}$ & $\begin{array}{l}3.5 \\
0.2 \\
0.2\end{array}$ & $\begin{array}{l}0.1 \\
1.1 \\
0.2\end{array}$ & 0.9 & 0.7 & $\begin{array}{l}0.2 \\
3\end{array}$ & $\begin{array}{l}0.7 \\
2.1\end{array}$ & $\begin{array}{l}0.3 \\
0.3\end{array}$ & & + & + & + & & & & + & \\
\hline $\begin{array}{l}\text { C. lineatus } \\
\text { C. perforotus } \\
\text { C. radiatus } \\
\text { C. stellaris var. symbolophorus } \\
\text { C. tabularis }\end{array}$ & 1.0 & 1.0 & 0.3 & 0.9 & 2.4 & 2.9 & 2.7 & 3.1 & & 1.7 & 7.9 & 1.1 & 0.9 & & $\begin{array}{l}+ \\
+ \\
+ \\
+\end{array}$ & & + & & & & & 1.7 \\
\hline $\begin{array}{l}\text { C. tabularis var. egregius } \\
\text { Cyclotella stylonum } \\
\text { Dactyliosolen antarcticus } \\
\text { Eucampia balaustium } \\
\text { Hemidiscus cuneiformis }\end{array}$ & 0.2 & & & & 0.6 & 0.3 & 0.4 & $\begin{array}{l}0.7 \\
0.1\end{array}$ & 3.5 & $\begin{array}{l}0.4 \\
0.6 \\
0.2\end{array}$ & $\begin{array}{l}0.2 \\
0.5 \\
0.2\end{array}$ & & 0.3 & + & & & & & & & & 0.6 \\
\hline $\begin{array}{l}\text { Lithodesmium undulatum } \\
\text { Navicula directa } \\
\text { N. trompii }\end{array}$ & & 0.3 & & & 0.2 & 0.3 & & & & & & & & & & & & & & & & 0.6 \\
\hline $\begin{array}{l}\text { Nitzschia angulata } \\
\text { N. bicapitata }\end{array}$ & 0.2 & $\begin{array}{l}1.0 \\
0.3 \\
\end{array}$ & 1.6 & $\begin{array}{l}1.5 \\
0.9 \\
\end{array}$ & $\begin{array}{l}1.4 \\
0.2 \\
\end{array}$ & $\begin{array}{l}1.4 \\
1.2 \\
\end{array}$ & $\begin{array}{l}1.3 \\
0.4 \\
\end{array}$ & $\begin{array}{l}3.3 \\
0.6 \\
\end{array}$ & 0.9 & $\begin{array}{l}0.9 \\
0.2 \\
\end{array}$ & $\begin{array}{l}0.5 \\
0.3 \\
\end{array}$ & $\begin{array}{l}0.4 \\
0.4\end{array}$ & $\begin{array}{l}0.3 \\
4.2 \\
\end{array}$ & & & & + & & & & & \\
\hline $\begin{array}{l}\text { N. curta } \\
N . \text { cylindrus } \\
N \text {. fraudulenta } \\
N . \text { grunowii } \\
N \text {. heimii } \\
\end{array}$ & 1.3 & 3.4 & 0.6 & 0.3 & $\begin{array}{l}0.2 \\
0.6\end{array}$ & $\begin{array}{l}0.9 \\
0.3 \\
0.6\end{array}$ & $\begin{array}{l}0.4 \\
0.4 \\
\end{array}$ & $\begin{array}{l}0.5 \\
0.1\end{array}$ & & 2.4 & 19.0 & 4.3 & 1.5 & & + & + & + & $\begin{array}{r}0.3 \\
11.8 \\
3.4 \\
14.3 \\
\end{array}$ & & 2.3 & & \\
\hline $\begin{array}{l}\text { N. kerguelensis } \\
\text { N. kolaczeckii }\end{array}$ & 27.9 & 38.9 & 51.3 & 22.1 & 4.4 & 46.3 & 48 & 70.4 & 85.7 & 82.7 & $\begin{array}{r}48.3 \\
0.2\end{array}$ & 1.8 & & & & & & & & & & \\
\hline
\end{tabular}




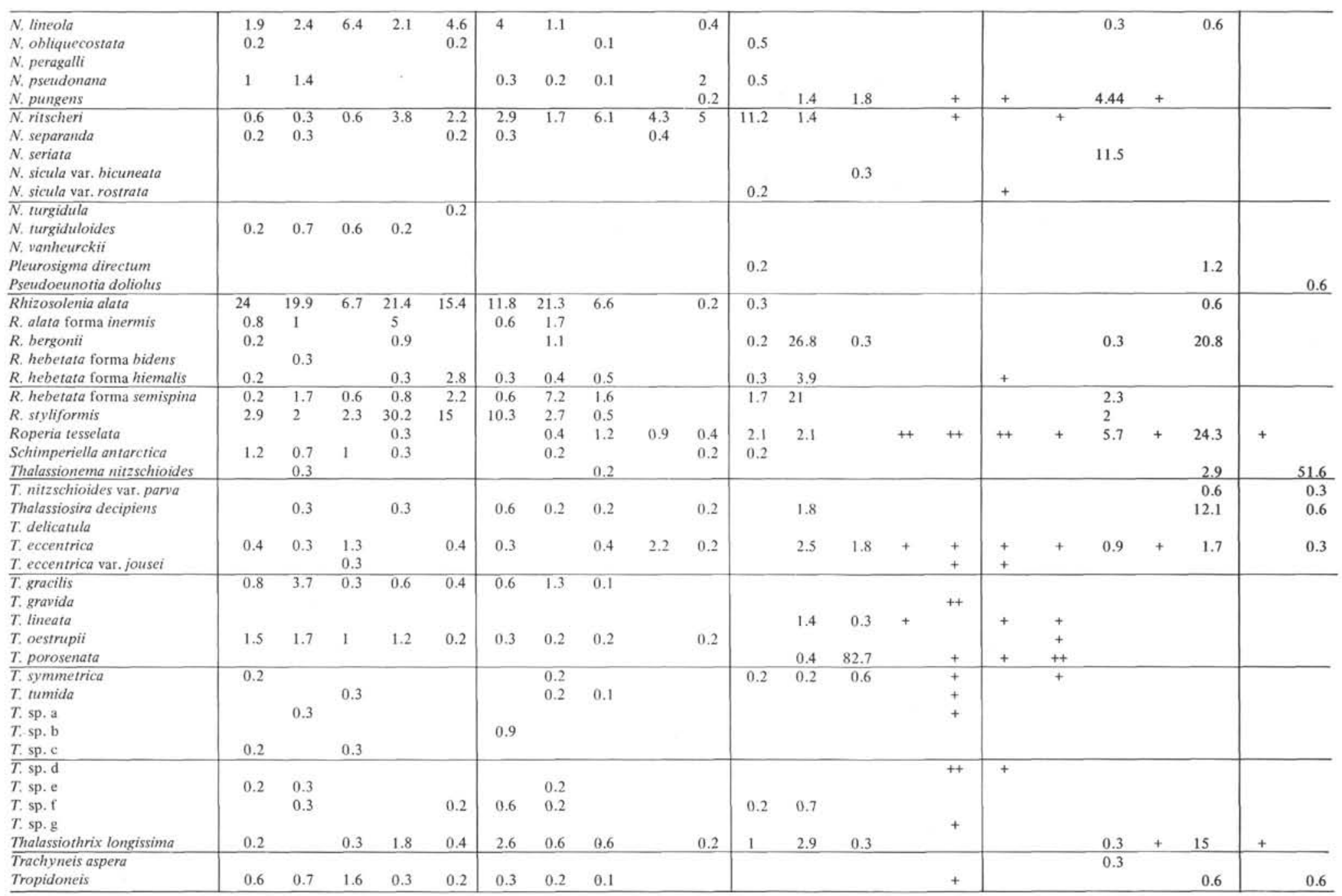




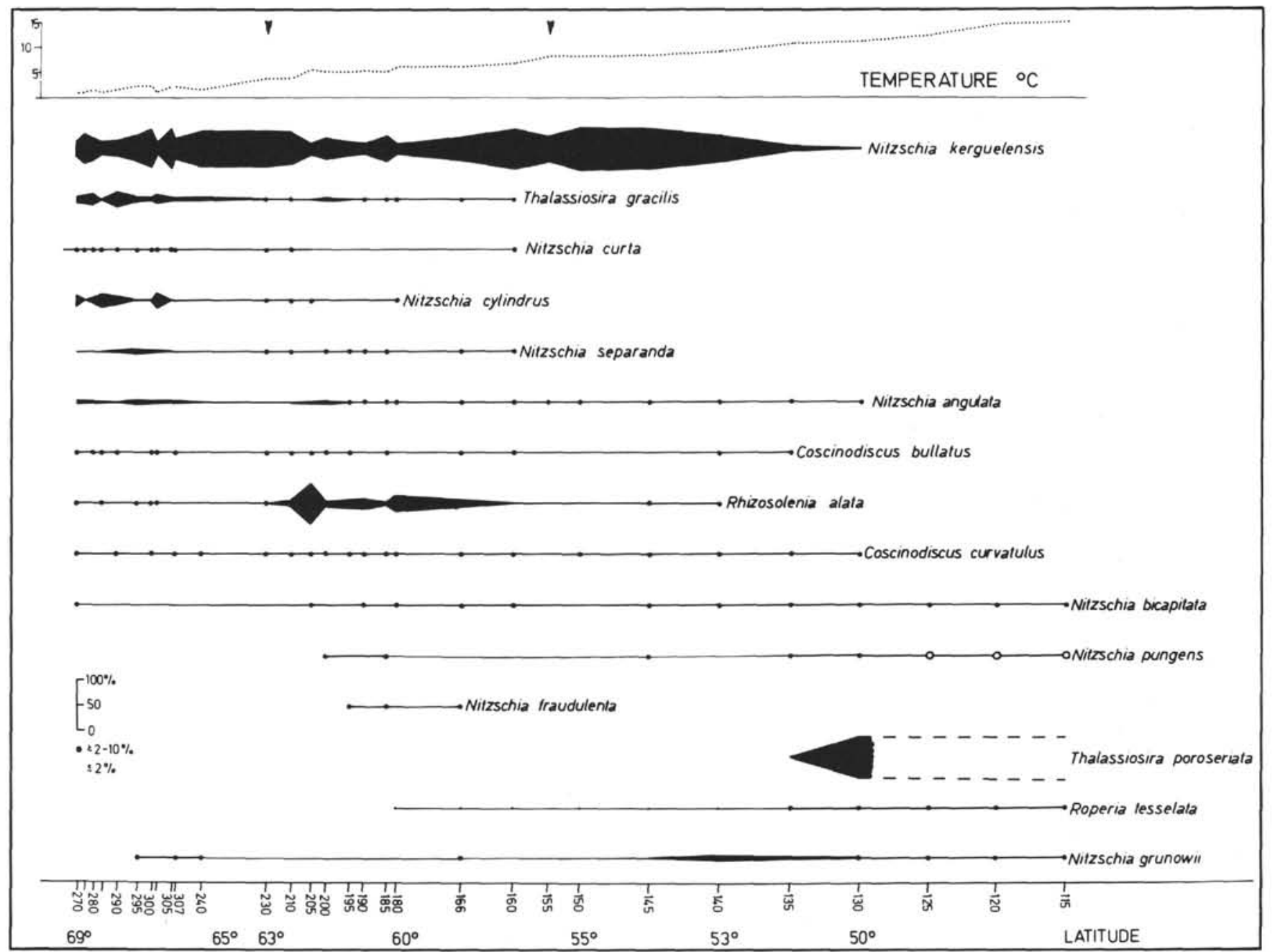

Figure 5. Percent abundance of important species of the total diatom assemblage at various stations. The arrows at the temperature line indicate the position of the Antarctic Convergence Zone (for explanation see text).

marginal apiculus in the middle. The hyaline line described by Hustedt (1930) connecting this apiculus with the central area is missing in the examined individual. Valve margin is finely striate: 20 striae in $10 \mu \mathrm{m}$.

\section{Genus AMPHORA Ehrenberg (1840)}

Amphora species 1 (aff. biarcuata Heiden and Kolbe, 1928) (Plate 11, Figure 14).

This species was found only at the northernmost station at $13^{\circ} \mathrm{S}$ latitude off the coast of Chile with $8.3 \%$ abundance. It has some resemblance to Amphora biarcuata which has been described by Heiden and Kolbe (1928) from Observatory Bay, Kerguelen. The present species differs by the much more convex dorsal side. The observed specimens were approximately $50 \mu \mathrm{m}$ long and 9-12 $\mu \mathrm{m}$ wide. The raphe is double curved because of slightly raised apices and central area. The axial area is very narrow. The number of transapical striae in $10 \mu \mathrm{m}$ is 19-20; striae show slightly radial arrangement.

\section{Amphora species 2}

(Plate 11, Figure 15)

This species was found only at the northernmost station at $13^{\circ} \mathrm{S}$ latitude, and in small numbers. The valves are semilanceolate with subcapitate ends. Specimens are 19-22 $\mu \mathrm{m}$ long and approximately 4 $\mu \mathrm{m}$ wide. The number of transapical striae is $22-23$ in $10 \mu \mathrm{m}$, with slight radial arrangement.
Genus ASTEROMPHALUS Ehrenberg (1845)

Asteromphalus heptactis (Bréb.) Ralfs (1861)

(Plate 4, Figures 22, 23)

Description: Hustedt (1930), p. 494, fig. 277.

Remarks: Valves are slightly convex, radially undulated, circular to oval, with diameters varying between 31 and $45 \mu \mathrm{m}$. The central area is eccentric, its diameter being $1 / 3$ of the valve diameter: $12-15$ $\mu \mathrm{m}$. The number of areolae in $10 \mu \mathrm{m}$ is 6-7. The valves are only thinly silicified. Only single individuals of this species were found north of $64^{\circ} \mathrm{S}$ latitude. Hustedt (1958) reported A. heptactis in plankton samples from the South Atlantic between $39^{\circ} \mathrm{S}$ and $41^{\circ} \mathrm{S}$ latitude, but in the intestine of Salpa fusiformis this species was found as far south as $69^{\circ} \mathrm{S}$ latitude; Manguin (1960) found the species in his samples from Adélie-Land. In general, the species is common in temperate waters (Heiden and Kolbe, 1928; Hustedt, 1930, 1958).

\section{Asteromphalus hookeri Ehrenberg (1844)}

(Plate 4, Figure 24)

Synonym: Asteromphalus robustus Castracane (1886).

Description: Hustedt (1930), p. 496-498, fig. 278.

Remarks: Valve diameter of the observed specimens is $90-104 \mu \mathrm{m}$. Except for the narrower ones, they generally have 8 hyaline rays with a width of 5.5-6.5 $\mu \mathrm{m}$. The hyaline central area occupies usually one half of the total diameter of the valve or less, and is $39-47 \mu \mathrm{m}$ in diameter. The number of areolae is $8-8.5$ in $10 \mu \mathrm{m}$; they are of uniform size over the whole valve. The species has been found in both the 


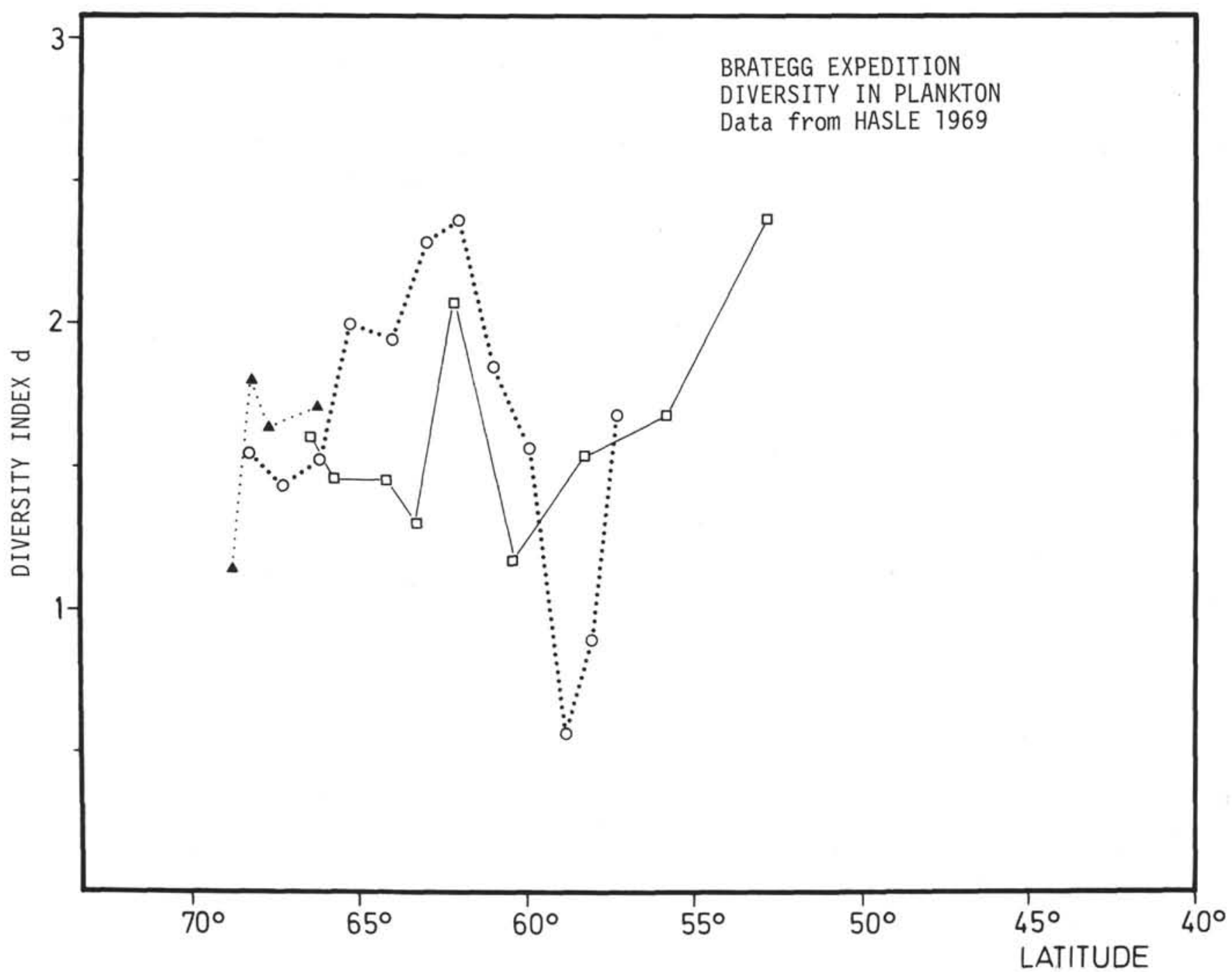

Figure 6. Diversity of phytoplankton samples (only diatom data) of Sections I (solid line) and II (dotted line) of the "Brategg" Expedition.

northern and southern hemisphere and is listed by most workers to occur in the Antarctic and Subantarctic regions: Van Heurck (1909, p. 43); Peragallo (1921, p. 75); Heiden and Kolbe (1928, p. 505); Manguin (1960, p. 257); Fenguelli and Orlando (1958, p. 133); Hasle (1969, p. 151); Balech and El-Sayed (1965, p. 109); and Hargraves $(1968$, p. 17-18). In the present study it was found in low percentages from the southern most station at $69^{\circ} \mathrm{S}$ to $53^{\circ} \mathrm{S}$ latitude in the north (i.e., in Antarctic as well as in Subantarctic waters), whereas Abbott (1972) found it to be fairly common only in the "Subantarctic" assemblage.

\section{Asteromphalus hyalinus Karsten (1905)}

(Plate 4, Figures 17-19)

Description: Hustedt (1959), p. 128, fig. 84-87.

Remarks: Valves convex, discoid with a valve diameter of 16-26 $\mu \mathrm{m}$. The number of hyaline rays is 4 or 5 ; they are $2-3 \mu \mathrm{m}$ wide and are curved, with the exception of one narrow and straight ray. The central area is eccentric and, in the 5-rayed specimens, is somewhat trapezoidal in shape. Its diameter is approximately one-half of the total valve diameter. The number of areolae in $10 \mu \mathrm{m}$ is 9-13.

This species was the most common Asteromphalus in the examined samples and had its greatest abundance in Antarctic waters. Karsten (1905) reported it only from $\left.\left.56^{\circ} 43\right) \mathrm{S}, 32^{\circ} 06\right) \mathrm{E}$. Hustedt (1958) records it from the Antarctic and Subantarctic zone in the southern Atlantic, as did Hargraves (1968); in the latter's samples this species was more abundant in the Antarctic than in the Subantarctic zone whereas Abbott (1972) found it only rarely in Subantarctic core tops of the eastern Antarctic part of the Indian Ocean.

\section{Asteromphalus parvulus Karsten (1905}

(Plate 4, Figures 20, 21)

Description: Hustedt (1958), p. 128, fig. 91

Remarks: Valves discoid, convex. Valve diameter 30-45 $\mu \mathrm{m}$. Hyaline central area occupies one-half or less of the total valve diameter. The number of hyaline rays is $5-7$, one much narrower than the others, the width of the broader rays being $2-2.8 \mu \mathrm{m}$. The number of areolae in $10 \mu \mathrm{m}$ is $9-10$.

This species has been found in Subantarctic as well as in Antarctic waters, but with greater abundance ( $4 \%$ of the diatom-flora) in Antarctic waters. Karsten (1905, p. 90) observed it at only one station, $59^{\circ} 16.3^{\prime} \mathrm{S}, 40^{\circ} 13.7^{\prime} \mathrm{E}$. Hustedt (1958, p. 128) found but one specimen at $51^{\circ} 29^{\prime} \mathrm{S}, 0^{\circ} 08^{\prime} \mathrm{W}$. Other authors report it in greater numbers: Hendey (1937, p. 270), from the Bellingshausen Sea and the Drake Passage; Frenguelli and Orlando (1958, p. 134); Manguin (1960, p. 258); Fukase (1962, p. 59); Cassie (1963, p. 6); Balech and El-Sayed (1965, p. 111), Fukase and El-Sayed (1965, p. 11); Hargraves (1968, p. $19)$; and Abbott (1972, p. 102). The latter observer found it to be common in the "Subantarctic" assemblage. 


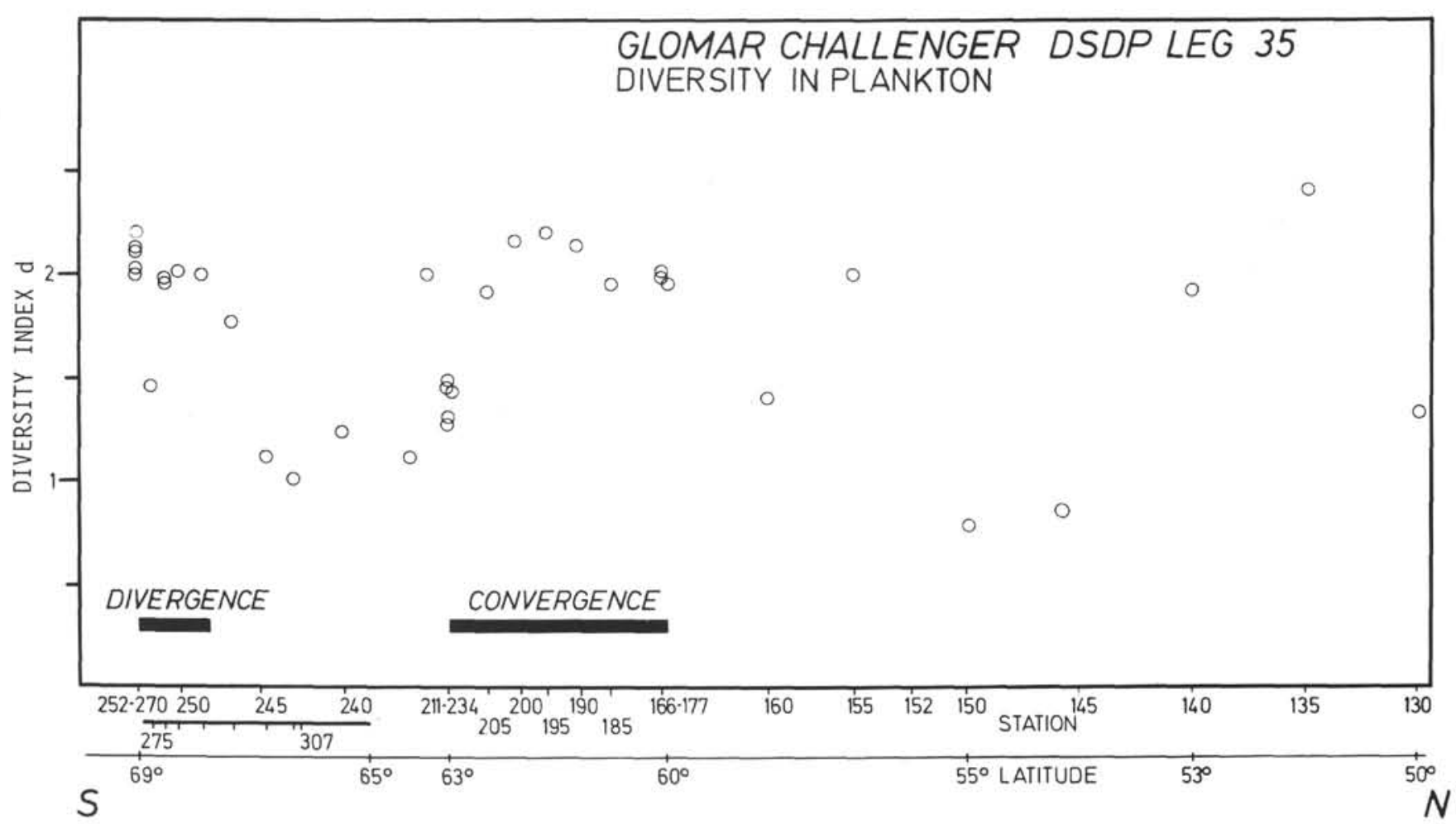

Figure 7. Diversity of diatom assemblages of Leg $35 \mathrm{D} / \mathrm{V}$ Glomar Challenger (for explanation see text).

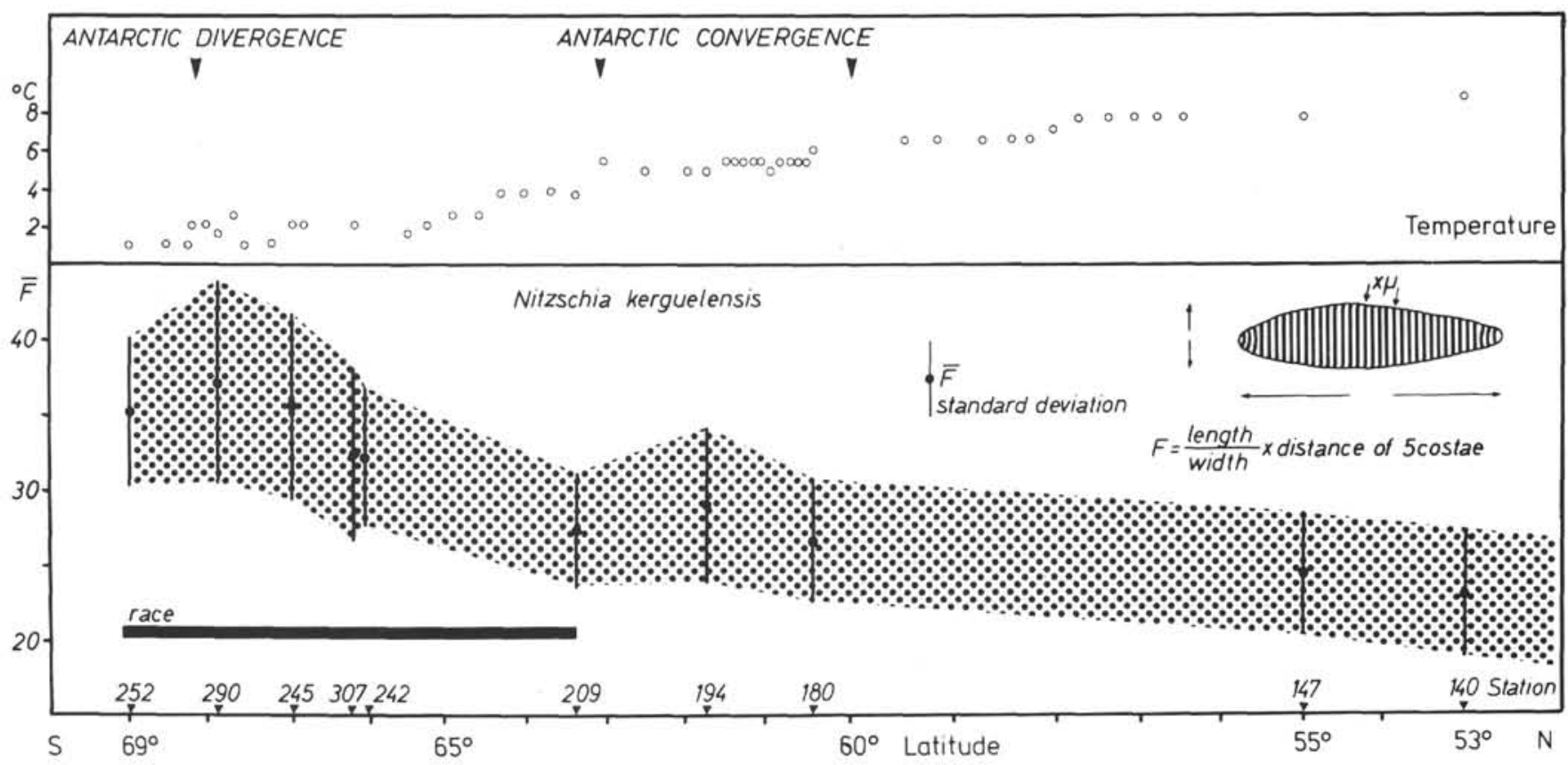

Figure 8. F values $=$ length $/$ width $\times$ width of 5 costae of Nitzschia kerguelensis in relation to the surface water temperatures and geographical latitude. Note Antarctic Divergence is situated south of $68^{\circ} \mathrm{S}$ latitude (for explanation see text).

Genus BACTERIASTRUM Shadbolt (1853)

Bacteriastrum hyalinum Lauder (1864) (No illustration)

Description: Hustedt (1930), p. 615-617, fig. 354

Remarks: This species was only found at the northernmost station at $13^{\circ} \mathrm{S}$ off the coast of Chile. This is in agreement with earlier records of it from temperate and warm waters (Hustedt, 1930, p. 616).
Genus BIDDULPHIA Gray (1821)

Biddulphia longicruris Greville (1859)

(Plate 11, Figure 16)

Description: Cupp (1943), p. 154-161, fig. 111.

Remarks: The species was found only north of $51.5^{\circ} \mathrm{S}$ latitude, in good agreement with Cupp (1943); Taylor (1966, p. 455); Hargraves (1968, p. 21); and Hendey (1937, p. 276). All authors describe Bid- 

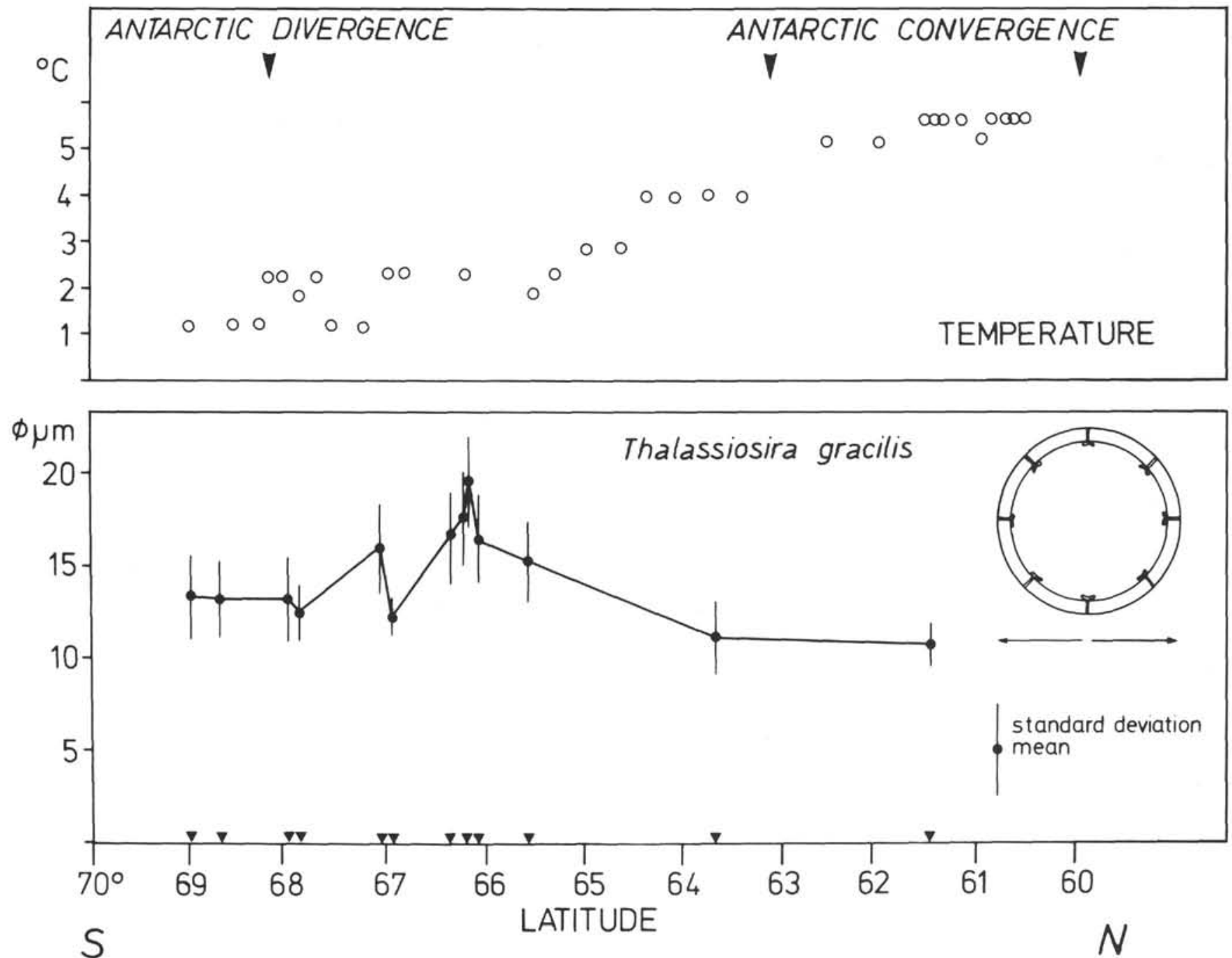

Figure 9. Diameter variations in Thalassiosira gracilis correlated to surface water temperatures and geographical latitude. Note Antarctic Divergence is situated south of $68^{\circ} \mathrm{S}$ latitude (for explanation see text).

dulphia longicruris as a form common in temperate and tropical seas. Hendey (1937) reported it to be frequent in the Peru Current.

Biddulphia sp.

(Plate 11, Figure 18)

\section{Genus CHAETOCEROS Ehrenberg (1844)}

Chaetoceros atlanticum forma bulbosum Hargraves (1968)

$$
\text { (No illustration) }
$$

Description: Hargraves (1968), p. 26-27, fig. 29-31.

Remarks: This forma of Chaetoceros atlanticum was counted separately from all the other Chaetoceros species because of its easy recognizability.

It was found only in the Antarctic zone, thus supporting $\mathrm{Har}$ graves' (1968) observation that it is a cold water form of the species.

\section{Genus CHARCOTIA M. Peragallo (1921)}

\section{Charcotia actinochilus (Ehrenberg) Hustedt (1958)}

(Plate 5, Figure 5)

Description: Hustedt (1958), p. 122-126, fig. 57-80.

Remarks: Individuals are $30-35 \mu \mathrm{m}$ in diameter; the number of areolae in the radial rows is $10-12$ in $10 \mu \mathrm{m}$ and the total number of marginal processes is 9-11.

The species was found in small numbers only in the Antarctic surface water samples. It has been reported to be restricted to the Antarctic zone (Van Heurck, 1909; Heiden and Kolbe, 1928; Hendey,
1937; Hustedt, 1958; Kozlova, 1964; Manguin, 1960; Hasle, 1969; Donahue, 1970; Abbott, 1972). Hargraves (1968 found it to be abundant in samples from the pack-ice zone.

\section{Genus COCCONEIS Ehrenberg (1838)}

Cocconeis costata var. antarctica Manguin (1960)

(Plate 11, Figure 12)

Description: Manguin (1960), p. 304, pl. 14, fig. 154-155 a-c.

Remarks: Only one individual of the species was found at station 75 , measuring $26 \mu \mathrm{m}$ in length and $16 \mu \mathrm{m}$ in width. Manguin (1960) described the species from littoral (?) plankton at Cap Margerie (Adélie Land).

\section{Cocconeis aff. placentula Ehrenberg (1838)}

(Plate 11, Figure 13)

Description: Hustedt (1959), p. 347-350, fig. 802-803.

Remarks: Only one single individual of a nonraphe-bearing frustule has been observed at station 175 .

\section{Genus CORETHRON Castracane (1886)}

\section{Corethron cirophilum Castracane (1886)} (No illustrations)

Description: Hendey (1937), p. 325.

Remarks: Worldwide in its distribution, this species is one of the most important in the southern oceans (Hustedt, 1958, p. 130). It is reported to occur in varying degrees of abundance from the Ant- 


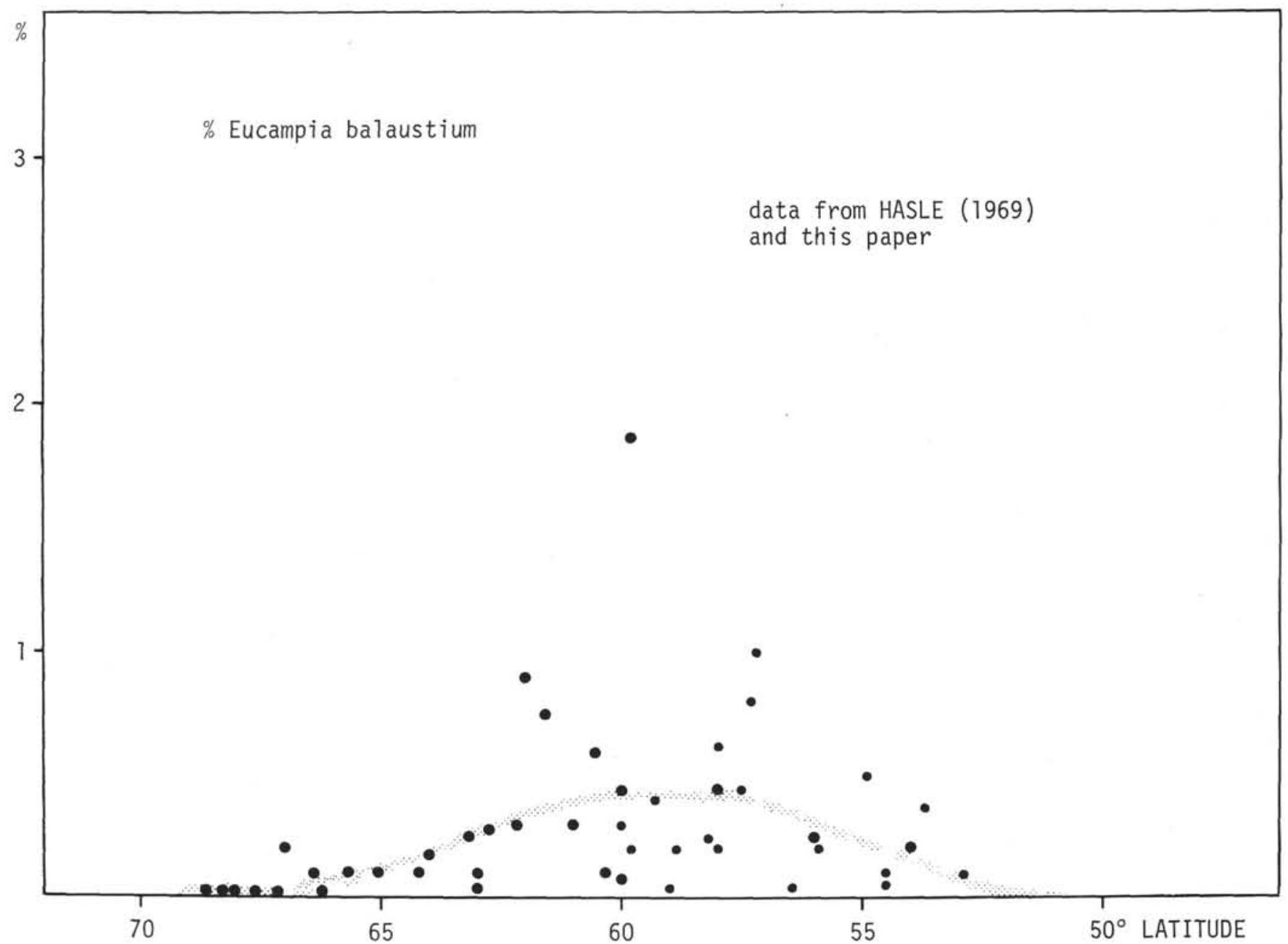

Figure 10. Percent abundance of total diatom assemblage of Eucampia balaustium in relation to the geographical latitude. Dotted line represents calculated mean values over $1^{\circ}$ of latitude.

arctic and Subantarctic region by Karsten (1905, p. 101, 104); Hendey (1937, p. 325$)$; Hustedt (1958, p. 130-131); Manguin (1960, p. 259260); Hargraves (1968, p. 39); and Hasle (1969, p. 151).

In the DSDP Leg 35 material it was present in varying degrees in nearly all samples. At station 285 it was found in large quantities.

\section{Genus COSCINODISCUS Ehrenberg (1838)}

\section{Coscinodiscus asteromphalus Ehrenberg (1844)} (No illustrations)

Description: Hustedt (1930), p. 452-545, fig. 250

Remarks: Valves discoid with a diameter of $71-175 \mu \mathrm{m}$. A central rosette with a central space is present. The loculi increase in size from the center outwards being largest halfway between the center and the margin; from there they decreased in size marginwards. Where they are largest, the number of loculi vary between $3-5$ in $10 \mu \mathrm{m}$. The interstitial meshes were present in great number and easily visible.

The species is cosmopolitan and neritic (Hendey, 1937). It is reported from the Arctic and Antarctic regions by Van Heurck (1909, p. 50); Peragallo (1921); Heiden and Kolbe (1928, p. 497); Hendey (1937); Frenguelli and Orlando (1958, p. 125); Hustedt (1958, p. 111112); Manguin (1960, p. 246); and Hargraves (1968, p. 39-40).

In this study it was only found north of $47^{\circ} \mathrm{S}$ and not in Antarctic samples. In one sample (station $1,13^{\circ} \mathrm{S}$ latitude), it was present in large numbers accountive for $15 \%$ of the rather scarce diatom flora.

\section{Coscinodiscus bullatus Janisch (1891)}

(Plate 6, Figures 11-14)

Description: Hustedt (1958), p. 112, pl. 4, fig. 26-28.

Remarks: Valves discoid and slightly concave. Valve diameter 27. $68 \mu \mathrm{m}$. Center of the valve slightly depressed. Loculi arranged in radial fascicles with the rows in the fascicles parallel to the middle row. The central loculi form an irregular cluster mostly surrounded by a hyaline ring. The number of loculi in $10 \mu \mathrm{m}$ was $9-12$. Marginal spines are generally distinct. Interstitial meshes within the fascicles are typical.

This species was found in small numbers throughout Antarctic and Subantarctic waters, with numbers increasing to the south; the highest percentage, $2.5 \%$ of the diatom flora, was reached between $63^{\circ}$ and $66^{\circ} \mathrm{S}$ latitude, but it occurs even in the northernmost sample $\left(13^{\circ} \mathrm{S}\right.$ latitude). Other authors, Hustedt (1958, p. 112); Hargraves (1968, p. 39-40); and Abbott (1972, p. 103), reported it as restricted to the Antarctic region. Only Jousé et al. (1962, p. 71) describe it from outside Antarctic regions.

\section{Coscinodiscus crenulatus Grunow (1884) (Plate 7, Figure 7)}

Description: Hustedt (1930), p. 411-412, fig. 219.

Remarks: Valve flat, discoid. Valve diameter, $38 \mu \mathrm{m}$. The loculi were of equal size on the valve face, their number being 10 in $10 \mu \mathrm{m}$. In the center of the valve is a cluster of irregularly arranged loculi surrounded by a thickened hyaline ring. The loculi are arranged in fascicles. A marginal labiate process (see Hasle and Fryxell, 1974) is always in continuation of the loculi rows running from the center to the margin. The margin is separated from the valve by a hyaline ring, which is irregularly interrupted by loculi. The valve margin is finely loculate (18 loculi in $10 \mu \mathrm{m}$ ).

Only one specimen of this species was found, at station $140\left(53^{\circ} \mathrm{S}\right.$ latitude).

Coscinodiscus crenulatus is a rare species known from warmer oceans: Kolbe (1954) (equatorial western Pacific); Hasle (1960) (tropical Pacific); Jousé et al. (1969). 
TABLE 3

"Natural" System of the Diatomaceae to Demonstrate Position of Treated Genera (after Simonsen, 1974)

\section{Class Bacillariophyceae}

I. Order Centrales

A. Suborder Coscinodiscineae

1. Family Melosiraceae Kützing, 1844

Corethron Castracane Dactyliosolen Castracane

2. Family Thalassiosiraceae Lebour, 1930

Cyclotella Küzing, Thalassiosira Cleve

3. Family Coscinodiscaceae Kotzing, 1844 Charcotia Peragallo Coscinodiscus Ehrenberg, Schimperiella Karsten

4. Family Hemidiscaceae Hendey, 1937 Actinocyclus Ehrenberg, Roperia Grunow, Hemidiscus Wallich

5. Family Stictodiscaceae Schütt, 1896

6. Family Heliopeltaceae H. L. Smith, 1872 Actinoptychus Ehrenberg

7. Family Asterolampraceae H. L. Smith, 1872 Asteromphalus Ehrenberg

8. Family Eupodiscaceae Kützing, 1849 B. Suborder Rhizosoleniineae

1. Family Pyxillaceae Schütt, 1896

2. Family Rhizosoleniaceae Petit, 1889

Rhizosolenia Ehrenberg, Lithodesmium Ehrenberg

3. Family Chaetoceraceae H. L. Smith, 1872

Bacteriastrum Shadbolt, Chaetoceros Ehrenberg

\section{Suborder Biddulphiineae}

1. Family Hemiaulaceae Heiberg, 1863 Eucampia Ehrenberg, Hemiaulus Ehrenberg

2. Family Biddulphiaceae Kützing, 1844 Biddulphia Gray

\section{Order Pennales}

A. Suborder Araphidineae

1. Family Diatomaceae Dumortier, 1822 Thalassionema Grunow

Thalassiothrix Cleve and Grunow

2. Family Protoraphidaceae Simonsen, 1970

B. Suborder Raphidoidineae

1. Family Eunotiaceae Kützing, 1844

C. Suborder Monoraphidineae

1. Family Achnanthaceae Kützing, 1844 Cocconeis Ehrenberg

D. Suborder Biraphidineae

1. Family Naviculaceae Kützing, 1844 Amphora Ehrenberg, Navicula Bory Pleurosigma W. Smith

Trachyneis Cleve, Tropidoneis Cleve

2. Family Epithemiaceae Grunow, 1860

3. Family Nitzschiaceae Grunow, 1860

Nitzschia Hassall, Pseudoeunotia Grunow

4. Family Surirellaceae Kützing, 1844

\section{Coscinodiscus curvatulus Grunow in A. Schmidt (1878)}

(Plate 6, Figures 3-10)

Description: Hustedt (1930), p. 406, fig. 214.

Remarks: Valves flat, discoid. Valve diameter 10-39 $\mu \mathrm{m}$. The areolae are grouped to slightly curved fascicles. The first row of each fascicle runs from the valve center to the margin; following rows run parallel and, accordingly, are shorter. At the end of the row running from the center to the margin a marginal process is always to be found.

The areolae vary in size from 6-16 in $10 \mu \mathrm{m}$; in the center of the valve they are sometimes irregularly distributed. The areolae decrease in size slightly from the center to the margin. The margin is finely striate with $18-22$ striae in $10 \mu \mathrm{m}$.

The species can be easily mistaken for Actinocyclus curvatulus, which is differentiated from Coscinodiscus curvatulus by a more or less articulate pseudonodulus and a broader marginal zone. Not even a faint indication of a pseudonodulus was observed in the examined specimens and the thickness of the margin in such small specimens as were found in the DSDP Leg 35 material, is of little specific value. All other authors who described the species from the Southern Oceans found only larger specimens $(35-80 \mu \mathrm{m})$ wherein a differentiation between it and $A$. curvatulus is easier.

Admittedly, in this study, some specimens included in Coscinodiscus curvatulus may be Actinocyclus curvatulus.

The species is described as being temperate (Hendey, 1937, p. 252; Hustedt, 1930, p. 409), and has been recorded in the Antarctic and Subantarctic zones by Van Heurck (1909); Hendey (1937, p. 252); Hustedt (1958, p. 112); Wood (1960); Manguin (1960, p. 247); Cassie (1963); and Hargraves (1968, p. 42). In the DSDP Leg 35 material Coscinodiscus curvatulus was found in samples from the Antarctic as well as the Subantarctic region. Higher frequencies occurred in the Antarctic Convergence zone and immediately south thereof where Coscinodiscus curvatulus makes up $2 \%-6 \%$ of the diatom flora; in the Antarctic zone it accounts for less than $0.5 \%$ of the diatom flora.

\section{Coscinodiscus kiitzingi Schmidt (1878)}

(Plate 7, Figure 2)

Description: Hustedt (1930), p. 398-399, fig. 209.

Remarks: Only one individual of this species was found at station $165\left(60^{\circ} \mathrm{S}\right.$ latitude). It has a diameter of $41 \mu \mathrm{m}$ and the number of areolae in $10 \mu \mathrm{m}$ is $7 ; 12$ areolae in $10 \mu \mathrm{m}$ occur within the marginal zone.

\section{Coscinodiscus lentiginosus Janisch (1878)}

(Plate 7, Figures 4-6)

Description: Hendey (1937), p. 248.

Remarks: Valves flat, discoid, diameter $20-53 \mu \mathrm{m}$. The areolae are dispersed more or less irregularly and loosely in the center of the valve, becoming ordered in radial lines and/or fascicles towards the margin. From the center to the valve margin the areolae are of constant size and decrease in size only near the valve margin. The largest areolae size is reached in some individuals at two-thirds of the way from the center to the margin. The number of areolae in $10 \mu \mathrm{m}$ varies between 7-11. The valve margin is fine-areolate with 10-13 areolae in $10 \mu \mathrm{m}$. Marginal spines are distinctly discernible as is a single prominent apiculus, which is typical for this species.

The species is characteristic of the Antarctic region and is also widespread in Subantarctic waters. Hendey (1937, p. 248); Hustedt (1958, p. 116); Hargraves (1968, p. 45); Hasle (1969, p. 151); Manguin (1960, p. 249); and Abbott (1972, p. 104) found it in small numbers even in the subtropical assemblage.

Coscinodiscus lentiginosus was found in DSDP Leg 35 samples in Antarctic and Subantarctic waters with a greater abundance in the Antarctic zone, but never reaching percentages much higher than $3 \%$ of the total diatom assemblage.

\section{Coscinodiscus lineatus Ehrenberg (1838)} (Plate 7, Figure 3)

Description: Hustedt (1930), p. 392, fig. 204.

Remarks: Valve diameter varies between $24-34 \mu \mathrm{m}$. The number of areolae is $7-8$ in $10 \mu \mathrm{m}$

A cosmopolitan species (Hustedt, 1930, p. 392) C. lineatas has a preference for warm to warm-temperate waters (Simonsen, 1974, p. 17). It has been reported from the Antarctic by Van Heurck (1909, p. 48); Peragallo (1921, p. 80 ); Heiden and Kolbe (1928, p. 482); Hendey (1937, p. 243); Frenguelli and Orlando (1958, p. 122); Manguin (1960, p. 249); Hargraves (1968, p. 46); and Abbott (1972, p. 104).

Coscinodiscus lineatus was found in DSDP Leg 35 samples in small numbers in the Antarctic, Subantarctic, and Subtropical waters.

\section{Coscinodiscus perforatus Ehrenberg (1844)}

$$
\text { (No illustration) }
$$

Description: Hustedt (1930), p. 445-449, fig. 245-246.

Remarks: One specimen of this species was found at station 105 $\left(41^{\circ} \mathrm{S}\right.$ latitude). The valve was extremely flat with a diameter of 122 $\mu \mathrm{m}$. The number of loculi is 5 in $10 \mu \mathrm{m}$, with 10 at the margin. Hyaline area and interstitial meshes, which Hasle and Fryxell (1974) found to be simple labiate processes are present. Coscinodiscus perforatus is described as being characteristic of temperate areas, but it 
has been also described by Fukase and El-Sayed (1965, p. 4) from Tierra del Fuego, and Hargraves (1968, p. 48) reports it from Subantarctic waters.

\section{Coscinodiscus radiatus Ehrenberg (1839)}

(Plate 7, Figure 1)

Description: Hustedt (1930), p. 420-421, fig. 225.

Remarks: Valves flat, discoid; valve diameter 41-49 $\mu \mathrm{m}$. Number of areolae are 4-6 in $10 \mu \mathrm{m}$ becoming gradually smaller at the margin (8-11). The areolae are arranged in radial rows with a tendency to an irregular arrangement. Interstitial meshes occasionally occur.

The species is considered to be common in all oceans (Hustedt, 1930, p. 421). Coscinodiscus radiatus was found in DSDP Leg 35 samples as far south as $47^{\circ} \mathrm{S}$ latitude. Hendey $(1937$, p. 250$)$ reported large numbers of robust individuals from the Peru Current off the coast of South America.

Coscinodiscus stellaris var. symbolophorus (Grunow) J $\phi$ rgensen (1905) (Plate 7, Figure 14)

Description: Hustedt (1930), p. 396-398, fig. 207-208.

Remarks: Individuals of this species were only found at station 120 $\left(52.5^{\circ} \mathrm{S}\right.$ latitude) in Subantarctic waters. It has been reported from the Antarctic and Subantarctic region by Hustedt (1958, p. 118-119); Hargraves (1968, p. 49-50); and Abbott (1972, p. 105). Coscinodiscus stellaris var. symbolophora and C. stellaris are common in temperate and warm waters although there is some indication that the variety occurs predominatly in Antarctic and Subantarctic regions (Grunow, 1884 , p. 82 ; Hargraves 1968 , p. $49-50)$. Hustedt (1958, p. 119) is inclined to combine these two forms because different valve structures can be found within one single cell, with one frustule having tangential structure and the other one having fasciculate structure. The number of areolae on the valve surface is highly variable. The observed individuals vary in valve diameter from 43 to $64 \mu \mathrm{m}$; the number of areolae in $10 \mu \mathrm{m}$ was 20 to 22 .

\section{Coscinodiscus tabularis Grunow (1884)}

(Plate 7, Figures 10-13)

Description: Hustedt (1958), p. 119, fig. 48-56.

Remarks: Valves discoid, flat, with a diameter of 13-39 $\mu \mathrm{m}$. Areolae are arranged in radial lines sometimes in fascicles; 5-10 areolae in $10 \mu \mathrm{m}$. There is always one large "areolae" positioned slightly eccentrically from the center; in some specimens the center is also eccentrically positioned. Typical is the hyaline area which separates the finely areolate marginal area, which has 14-18 areolae in $10 \mu \mathrm{m}$. The number of marginal labiate processes was 5-14.

The species is confined to the Antarctic and Subantarctic water masses. It is reported to occur in variable numbers by Heiden and Kolbe (1928); Hustedt (1958); Manguin (1960); Cassie (1963); Hargraves (1968); Hasle (1969); and Abbott (1972). Abbott found this species to occasionally occur in his subtropical diatom assemblage.

Coscinodiscus tabularis was found in DSDP Leg 35 samples in small quantities in the Antarctic region. Its abundance increased in the Antarctic Convergence zone and even more abundantly, up to $20 \%$ of the total diatom flora, in the Subantarctic zone.

\section{Coscinodiscus tabularis var. egregius (Rattray) Hustedt (1930)}

(Plate 7, Figures 8, 9)

Description: Hustedt (1930), p. 428, fig. 230b.

Remarks: The variety differs from the species in possessing a closed net of areolae with no hyaline ring in the central part. The hyaline ring at the margin is smaller than in the species. The eccentric central process is present. Valve diameter ranges between 23 to 38 $\mu \mathrm{m}$. The number of marginal labiate processes varies from 6 to 12 . The number of areolae is $7-12$ in $10 \mu \mathrm{m}$ and, in the marginal zone, 1418 in $10 \mu \mathrm{m}$.

The variety had the same distribution as the species, but is not as abundant.

\section{Genus CYCLOTELLA Kutzing (1833)}

Cyclotella stylorum Brightwell (1860) (No illustration)

Description: Hustedt (1930), p. 348, fig. 179.
Remarks: One specimen of this species, mainly distributed in warmer waters, was found at the northernmost Station, $13^{\circ} \mathrm{S}$ latitude, off the coast of Chile.

\section{Genus DACTYLIOSOLEN Castracane}

\section{Dactyliosolen antarcticus Castracane (1886) (Plate 11, Figure 11)}

Description: Hendey (1937), p. 323-324, pl. VI, fig. 1-3.

Remarks: This species is widely distributed in the northern and southern hemispheres (Hendey, 1937). It was reported from the Subantarctic and Antarctic waters by Heiden and Kolbe (1928); Hustedt (1958); Manguin (1960, p. 261); Hargraves (1968, p. 54, 55); and Hasle (1969, p. 151).

In the DSDP Leg 35 plankton material, some specimens of the species were found in the Subantarctic region.

\section{Genus EUCAMPIA Ehrenberg (1839)}

\section{Eucampia balaustium Castracane (1886)} (Plate 5, Figures 7-9)

Description: Hendey (1937), p. 285-286, pl. XIII, fig. 8-10.

Remarks: The species is variable in length with apical axes varying from $27-43 \mu \mathrm{m}$. Some of the valves are coarsely structured with 4-5 loculi in $10 \mu \mathrm{m}$, or more finely structured with about 8 loculi in 10 $\mu \mathrm{m}$.

This diatom is characteristic of the Antarctic and Subantarctic regions [Heiden and Kolbe (1928, p. 535); Hendey (1937, p. 286); Hustedt (1958, p. 137); Manguin (1960, p. 292); Hasle (1969, p. 151); Hargraves (1968, p. 55-56); and Abbott (1972, p. 106). In Leg 35 collections it was found to be more frequent in Subantarctic waters and in the Antarctic Convergence than in Antarctic waters.

\section{Genus HEMIDISCUS Wallich (1860)}

\section{Hemidiscus cuneiformis Wallich (1860)} (Plate 11, Figure 17)

Description: Simonsen (1972), p. 267, fig. 7-11.

Remarks: The species is recognized to be a tropical and subtropical, species but, repeatedly, single specimens have been reported from the Subantarctic zone: Heiden and Kolbe (1928, p. 547 , as Euodia gibba and Euodia inornata); Hendey (1937, p. 265); Taylor (1966, p. 455); Hargraves (1968, p. 66); Hasle (1969, p. 151); and Abbott $(1972$, p. 110).

In the present material two specimens were found, one at station $201\left(62.5^{\circ} \mathrm{S}\right.$ latitude) and the other at station $14053^{\circ} \mathrm{S}$ latitude).

\section{Genus LITHODESMIUM Ehrenberg (1840)}

\section{Lithodesmium undulatum Ehrenberg (1840)} (Plate 5, Figure 6)

Description: Hustedt (1930), p. 789-791, fig. 461.

Remarks: Lithodesmium is a neritic species common in temperate waters (Hustedt 1930, p. 791; Cupp, 1943, p. 151). Three specimens were only found at the northernmost station 1 ( $13^{\circ} \mathrm{S}$ latitude).

\section{Genus NAVICULA Bory (1824)}

\section{Navicula directa (W. Smith) Ralfs in Pritchard (1861) (Plate 14, Figure 7)}

Description: Cleve (1895), v. 27, no. 3., p. 27.

Remarks: Valves lanceolate, with narrow axial area and small central area. Length $23-63 \mu \mathrm{m}$, width 6-9 $\mu \mathrm{m}$. Number of transapical striae is $9-13$ in $10 \mu \mathrm{m}$.

A cosmopolitan species, more common in colder waters, it occurs sporadically in the Antarctic region and the Antractic Convergence zone. Other authors who reported the species from the Subantarctic and Antarctic waters are Manguin (1960, p. 312); Heiden and Kolbe (1928, p. 698); Hargraves (1968, p. 71); and Abbott (1972, p. 113).

\section{Navicula trompii Cleve (1900)}

(Plate 14, Figure 9)

Description: Cleve (1900), p. 932. 
Remarks: This species was found to occur sporadically within the Antarctic region only, in good accordance with the reports of Heiden and Kolbe (1928, p. 625); Hustedt (1958, p. 147); Hargraves (1968, p. 74); and Hasle (1969, p. 151). It seems to be restricted to the colder waters of the southern hemisphere, commonly associated with packice (Hargraves, 1968).

\section{Nitzschia angulata (O'Meara) Hasle (1972)}

(Plate 1, Figures 17-39)

Description: Hasle (1965), p. 24-26; pl. 1, fig. 6; pl. 4, fig. 19; pl. 9, fig. 1-6.

Remarks: The length varies from $8-53 \mu \mathrm{m}$, the width from $7-14$ $\mu \mathrm{m}$. Length and width are correlated in Figure 11. The number of transapical costae is $10-20$ in $10 \mu \mathrm{m}$, the number of poroids in $10 \mu \mathrm{m}$ varies between 15 and 24

$N$. angulata is reported to be circumpolar from Antarctic and Subantarctic waters; Hasle (1969); Castracane (1886); Manguin (1960); Heiden and Kolbe (1929); Frenguelli and Orlando (1958); Frenguelli (1960); Manguin (1960); Kozlova (1962, 1964); and Hargraves (1968). The northernmost occurrence is reported by Hustedt (1958), who found it in plankton as far north as $49^{\circ} \mathrm{S}$ latitude. $N$. angulata was one of the common species in DSDP Leg 35 samples. It reached its maximum abundance of more than II\% at the Divergence zone where, also, it reached its greatest length. It was not found north of station 130. Hasle $(1969,1965)$ and Hargraves (1968) reported it from the undersurface of pack-ice.

\section{Nitzschia bicapitata Cleve (1900)}

(Plate 3, Figures 27-29)

Description: Hustedt (1958), p. 169, fig. 185-190.

Remarks: The size of the specimens collected on Leg 35 varies between $16.2-20 \mu \mathrm{m}$ in length and $4.5-5.5 \mu \mathrm{m}$ in width. The number of costae in $10 \mu \mathrm{m}$ is $21-26$ and the number of keel-punctae is $12-16$ in 10 $\mu \mathrm{m}$.

The list of localities where this species has been found, as reported by Hustedt $(1958$, p. 169$)$ and Hasle $(1960$, p. 21$)$, shows the worldwide distribution of Nitzschia bicapitata and its great temperature tolerance. It was reported by Hustedt $(1958)$ and Hasle $(1969,1964)$ from the Subantarctic zone with a maximum abundance just north of the Antarctic zone. Hargraves (1968) found it in the Antarctic zone. $N$. bicapitata was observed in DSDP Leg 35 samples from the Subantarctic and Antarctic region as well, but with a maximum abundance in the Antractic Convergence zone and the Subantarctic region. In the Antarctic it was found to occur only sporadically and as far south as southernmost station at $69^{\circ} \mathrm{S}$ latitude.

\section{Nitzschia curta (Van Heurck) Hasle (1972)}

(Plate 4, Figures 5-9)

Description: Hasle (1965), p. 32-33, pl. 12, fig. 2-5.

Remarks: Examined specimens are $15-43 \mu \mathrm{m}$ long, 5-6.2 $\mu \mathrm{m}$ wide (Figure 12) and have 11-16 costae in $10 \mu \mathrm{m}$. Valves were characterized by the hetoropole apical axis and the curved transapical costae near the apices. Transitional forms between $N$. curta and $N$. cylindrus were found; heteropolarity of the apical axis was used to distinguish between the two species whereas length, width, and number of transapical costae overlap in the two species.

$N$. curta is indigenous in the Antarctic zone with increased abundance southward to the Antarctic continent: Hasle (1959, 1965); Manguin (1960); Kozlova (1964); and Cassie (1963). Hasle (1959, 1965); Van Heurck (1909); Hendey (1937); and Hargraves (1968) found it in samples from the pack-ice and shelf-ice zone. Hustedt (1958) observed the species in samples from Antarctic waters north of the subtropical zone in the South Atlantic.

$N$. curta was observed in DSDP Leg 35 samples at stations in the Antarctic zone southward of station 210 , with increasing abundance to the south; it accounts for $5 \%$ of the diatom flora at the southernmost station 252 .

\section{Nitzschia cylindrus (Grunow) Hasle (1972$$
\text { (Plate 4, Figures 10-15) }
$$

Description: Hasle (1965), p. 34-37, pl. 12, fig. 6-12

Remarks: Studied specimens have an apical axis of $3.2-20 \mu \mathrm{m}$, with widths of $1.5-5 \mu \mathrm{m}$. The number of transapical costae in $10 \mu \mathrm{m}$ varies from 13-20. Length and width were positively correlated (Figure 13).

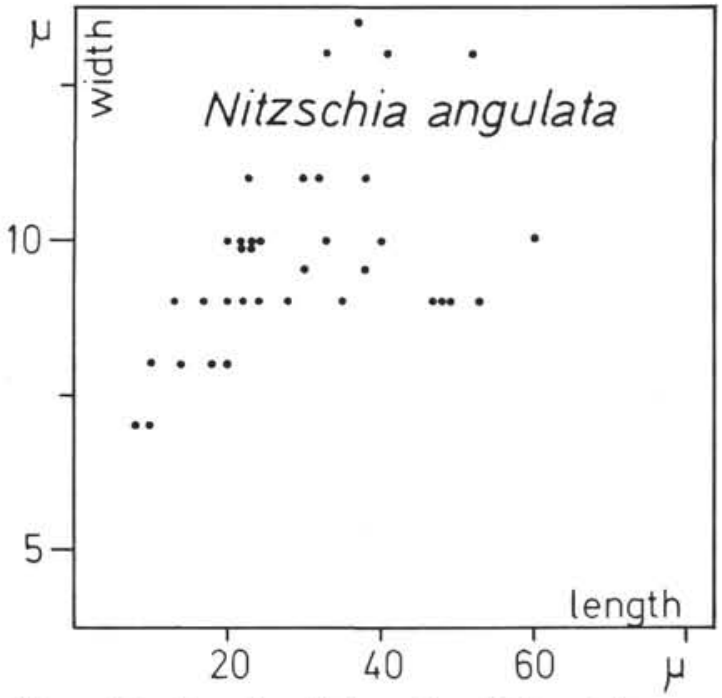

Figure 11. Length-width ratio of Nitzschia angulata.

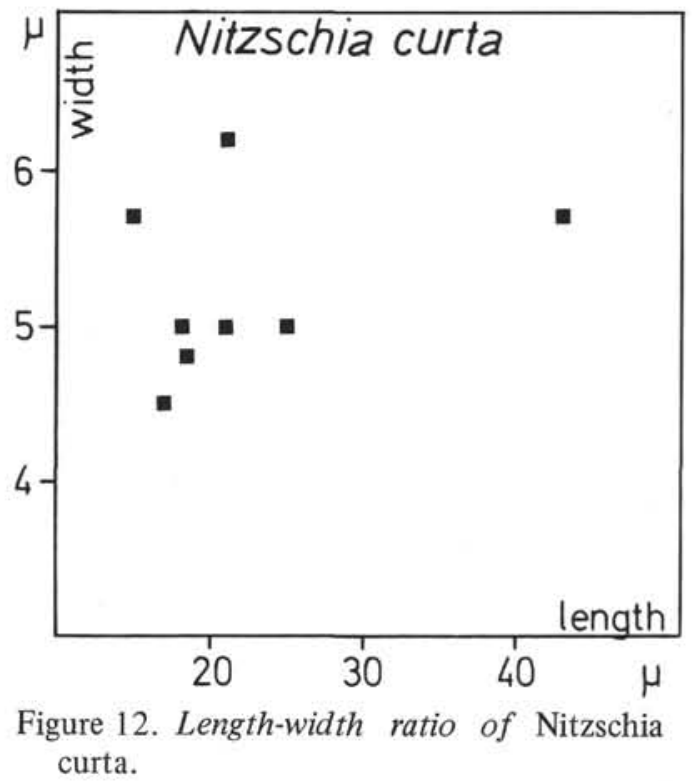

The smaller individuals were collected (plankton net $22 \mu \mathrm{m}$ meshsize) because they were included in fecal pellets. $N$. cylindrus was characterized by Hasle (1965) as being a bipolar cold water species. Peragallo (1921), Werff (1954), Frenguelli and Orlando (1958), Manguin (1960), Jousé et al. (1962), Kozlova (1962), and Hustedt (1958) found it abundant in Antarctic waters and less abundant as far north as $46^{\circ} \mathrm{S}$ latitude in the south Atlantic. Hasle $(1959,1965)$ record it in great abundance in "Brategg" samples from the Antarctic zone of the South Pacific with increasing abundance towards the south and equally abundant in pack-ice samples (Hasle 1969, 1965; Hargraves 1968).

$N$. cylindrus was found in the DSDP Leg 35 samples to have a similar occurrence as $N$. curta increasing in number to the south. The highest numbers, up to $30 \%$ of the total diatom flora, were found at station 300 .

\section{Nitzschia fraudulenta Cleve (1897)}

(Plate 3, Figure 17)

Description: Hasle (1964), pl. 6, fig. 2; Hasle (1965), pl. 1, fig. 2, 3; pl. 4 , fig. $8-10$; pl. 6 , fig. $5-10$; pl. 8 , fig. 1-3.

Remarks: The frustules have the typical spindle shape. Length varies from 75-110 $\mu \mathrm{m}$, width from 5.5-7.5 $\mu \mathrm{m}$. Keel-punctae and transapical ribs are present in approximately equal numbers: keel- 


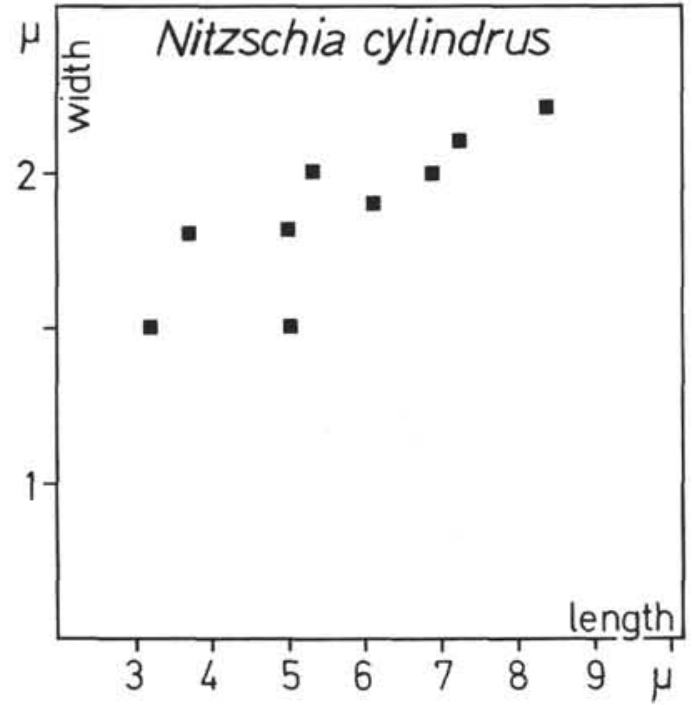

Figure 13. Length-width ratio of Nitzschia cylindrus.

punctae 19-24 in $10 \mu \mathrm{m}$, and transapical ribs $21-24$ in $10 \mu \mathrm{m}$. The keelpunctae were more widely spaced in the middle. Pseudonodulus was present.

Specimens were found in greatest abundance only north of the Antarctic Convergence at station 101. Isolated specimens were found at stations 170, 185, and 195. Most probably these specimens are allochthonous.

Hasle (1972) quotes that $N$. fraudulenta has been found off the coast of Chile from about $44^{\circ} \mathrm{S}$ latitude. Neither Hustedt (1958) nor Hargraves (1968) reported the species.

\section{Nitzschia grunowii (Cleve) Hasle (1972) \\ (Plate 2, Figures 1-5)}

Description: Hasle (1965), p. 11-14; pl. 1, fig. 15-19.

Remarks: The observed length is $24-63 \mu \mathrm{m}$, the breadth $7-10 \mu \mathrm{m}$, and the number of costae $11-18$ in $10 \mu \mathrm{m}$, the costae forming stronger "punctae" at one margin (after Hasle, 1965) and are interpreted as keel-punctae. The intercostal membranes are poroid (TEM-investigations), the poroids not being visible in normal transmitted light microscopy. Specimens in which the two marginal swellings in the middle of the valves were not more widely spaced than the others were found associated with $N$. grunowii.

All specimens were weakly silicified; small forms are spindleshaped. With increasing length, the margins become more linear than the middle area.

$N$. grunowii was found in the southern hemisphere only in small numbers by Peragallo (1921), Frenguelli and Orlando (1958), and Wood (1960) from Antarctic and Subantarctic waters, by Cassie (1963) from New Zealand waters, by Krasske (1941) from Chilean coastal waters, and by Frenguelli (1938) and Frenguelli and Orlando (1959) from Argentine inshore and offshore waters.

The species was found in DSDP Leg 35 samples in both Subantarctic and Antarctic waters from $41.7^{\circ} \mathrm{S}$ latitude to $67.5^{\circ} \mathrm{S}$ latitude (stations 101, 140, 235, 295, 307, and 170).

\section{Nitzschia heimii Manguin (1960)}

(Plate 3, Figures 21-23)

Description: Hasle (1964), pl. 2, fig. 3; pl. 4, fig. 7; Hasle (1965), pl. 1 , fig. 6,7 ; pl. 10, fig. 9-13; pl. 11, fig. 1-7.

Synonyms: Pseudonitzschia heimii Manguin (1957), p. 131, pl. 6, fig. 43; N. pacifica Cupp (sensu Hustedt) Hustedt (1958), p. 177, pl. 13, fig. 193, 194.

Remarks: The valves are linear-lanceolate with obtuse apices. Most specimens have a constriction near the apices. Length varies from 75 to $150 \mu \mathrm{m}$, and breadth from 5 to $6.5 \mu \mathrm{m}$. The number of transapical costae in $10 \mu \mathrm{m}$ is $18-24$, where the number of keel- punctae in $10 \mu \mathrm{m}$ is $11-16$. The keel is eccentric. The two keel-punctae in the middle are more widely spaced.

$N$. heimii is widely distributed and occurred in DSDP Leg 35 Samples from stations $101,130,155,160,170,175,185,190,195,201$, $205,210,215,220,225,235$, between $41.7^{\circ} \mathrm{S}$ to $63.5^{\circ} \mathrm{S}$ latitude. This distribution agrees well with the records of Hustedt (1958) who found $N$. heimii in the South Atlantic plankton from $44^{\circ} \mathrm{S}$ to $61^{\circ} \mathrm{S}$ latitude, and with the data of Manguin (1960) from the area south of Tasmania.

The greatest abundance of Leg 35 samples occurs between 61.5 to $63^{\circ} \mathrm{S}$ latitude within the Antarctic Convergence zone. Hargraves (1968) and Manguin (1960) found N. heimii in greater abundance immediately south of the Antarctic Convergence but Hustedt (1958) found the greatest abundances at $51^{\circ} \mathrm{S}$ latitude, and Hasle (1969) recorded maximum occurrences just north of the Antarctic Convergence. $N$. heimii appears to have its maximum occurrence close to the Antarctic Convergence.

\section{Nitzschia kerguelensis (O'Meara) Hasle (1972)}

(Plate 2, Figures 19-30)

Description: Hasle (1965), p. 14-18, pl. 4, fig. 11-18; pl. 7, fig. 9 (as Fragilariopsis kerguelensis O'Meara).

Remarks: The length and breadth values are $14-83 \mu \mathrm{m}$ and $4.5-12$ $\mu \mathrm{m}$, respectively. The number of poroids in the intercostal membranes varies from 7 to 13 in $10 \mu \mathrm{m}$ and the number of transapical costae in $10 \mu \mathrm{m}$ varies from 5 to 8 . Valves are straight to slightly oblique, sometimes slightly curved near the apices. Valves are strongly silicified, neither keel-punctae nor a canal-raphe was observed. Shape is variable, the smaller specimens are elliptical with broadly rounded apices. The medium-sized specimens are predominantly oval often with a pronounced heteropolarity of the apical axis; extremely long specimens are narrower. Larger specimens characteristic of lower water temperatures.

$N$. kerguelensis is one of the most abundant forms in all Antarctic and Subantarctic samples occurring as far north as $52^{\circ} \mathrm{S}$ latitude.

\section{Nitzschia kolaczeckii Grunow (1867)}

(Plate 3, Figure 16)

Description: Hasle (1960), p. 24, fig. 50 a-c.

Remarks: A single specimen was found at station 140, with a length of $76 \mu \mathrm{m}$, and a width of $10 \mu \mathrm{m}$. In addition it has 15-16 transapical costae in $10 \mu \mathrm{m}, 12$ poroids in $10 \mu \mathrm{m}$, and 8-9 keel-punctae in $10 \mu \mathrm{m}$. This harmonizes with the reports of Grunow $(1877, \mathrm{p} .173)$ and Hargraves (1968). Nitzschia kolaczeckii is known from subtropic to tropic waters (see list in Hasle, 1960). Some authors described it as a littoral form (Grunow, 1867, 1877; Petit, 1889; Boyer, 1927).

\section{Nitzschia lineola Cleve (1897)}

(Plate 3, Figures 24-26)

Description: Hasle (1965), pl. 2, fig. 4, 5; pl. 12, fig. 15, 16, 17-21; pl. 13, fig. 11-14; pl. 14, fig. 1-3.

Synonym: Nitzschia barckleyi Hustedt (1952).

Remarks: The length of the observed specimens in Leg 35 samples varies widely from 60 to $110 \mu \mathrm{m}$ where the width was quite constant at $2-2.5 \mu \mathrm{m}$ (see Figure 14).

Cleve (1897) recorded the species to occur as far south as $45^{\circ} \mathrm{S}$ latitude in the Atlantic and the Indian oceans and interpreted it as being a temperate-oceanic species. Hasle (1965) found it to be abundant in the "Brategg" samples, and Hargraves (1968) reported it in the Antarctic region of the Pacific Ocean occurring occasionally in the pack-ice (temperature $-0.8-+3.3^{\circ} \mathrm{C}$ ). In the DSDP Leg 35 planktonmaterial it occurred from $53^{\circ} \mathrm{S}$ latitude to the southernmost station at $69^{\circ} \mathrm{S}$ latitude, with a maximum abundance at $66.8^{\circ} \mathrm{S}$ latitude and in the region of the Antarctic Convergence at $60-61^{\circ} \mathrm{S}$ latitude. It was not found north of $53^{\circ} \mathrm{S}$ latitude.

\section{Nitzschia obliquecostata (Van Heurck) Hasle (1972)}

(Plate 2, Figures 15-18)

Description: Hasle (1965), p. 18-20, pl. 7, fig. 2-7.

Remarks: Individuals vary from $62-87 \mu \mathrm{m}$ in length and $7-9 \mu \mathrm{m}$ in width. The number of transapical costae is $6.5-9$ in $10 \mu \mathrm{m}$, and the number of poroids 11-14 in $10 \mu \mathrm{m}$. Valves are linear-lanceolate with an expansion over the middle of the valves; apical axis is heteropol. Transapical costae are slightly to obviously oblique and sometimes 


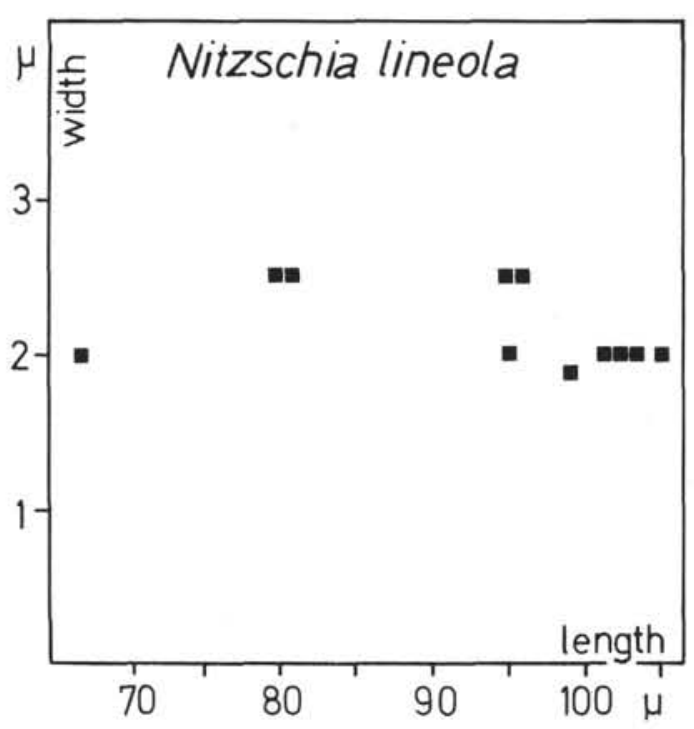

Figure 14. Length-width ratio of Nitzschia lineola.

curved at the apices. Almost all specimens show two rows of poroids within the intercostal membranes; occasionally one or three rows occur. Although the number of poroid rows corresponds to the data given by Hasle (1965), they were intermediate between $N$. kerguelensis and $N$. obliquecostata. All specimens with an eccentrical raphe are here combined with $N$. obliquecostata. The number of keelpunctae is the same as the number of transapical costae; in some individuals their arrangement is irregular, but no indication of a greater distance between the two middle punctae was observed.

Solitary specimens were found in both Antarctic and Subantarctic waters at stations $140,195,175,252,265$, and 305 . Hustedt (1958) observed a similar wide distribution as did Jousé et al. (1962); Hasle (1969, 1965); and Hargraves (1968). The latter found $N$. obliquecostata in samples from the pack-ice. Fritsch (1912), Frenguelli and Orlando (1958), and Kozlova (1962) found the species restricted to the Antarctic coastline and to the ice frontier.

\section{Nitzschia peragallii Hasle (1965)}

(Plate 2, Figures 12-14)

Description: Hasle (1965), p. 41-42; pl. 9, fig. 11, 12; pl. 15, fig. 1-5.

Remarks: Specimens range from $63-84 \mu \mathrm{m}$ long, and from $6.5-8$ $\mu \mathrm{m}$ wide; they have 6-7.5 transapical costae and $12-16$ poroids in 10 $\mu \mathrm{m}$. Keel-punctae are present in numbers equal to the costae, with no indication that the middle keel-punctae are separated by a distance greater than the others.

This species differs from $N$. obliquecostata by its narrower width and parallel sides.

It was found on Leg 35 only at the southernmost stations south of the Antarctic Convergence. The same findings were reported by Hasle (1965) who found it also in net samples from the southernmost "Brategg" stations and in pack-ice.

\section{Nitzschia pseudonana Hasle (1972)}

(Plate 2, Figures 6-11)

Description: Hasle (1965), p. 22-24, pl. 1, fig. 7-14; pl. 4, fig. 20, 21.

Remarks: Specimens are 6.1-20 $\mu \mathrm{m}$ long, 3-8 $\mu \mathrm{m}$ wide, and have 18-24 transapical costae and keel-punctae in $10 \mu \mathrm{m}$.

N. pseudonana was characterized by Hasle (1965) as a cosmopolitan species occurring in small numbers at the southernmost "Brategg" stations and in greater quantities in the Subantarctic zone. In DSDP Leg 35 plankton samples $N$. pseudonana was found south of station 140 with increasing numbers to the south. The greatest frequency was 5\%, attained in the Antarctic Divergence zone.

\section{Nitzschia pungens Grunow in Cleve and MöHler (1882)} (Plate 3, Figure 18)

Description: Hasle (1965), p. 12-14, pl. 1, fig. 4, 5; pl. 5, fig. 7-9; pl. 6 , fig. 3 ; pl. 7 , fig. $1-8$.
Remarks: The valves are linear-lanceolate with pointed ends; valve ends show considerable overlap. The observed specimens are 50-130 $\mu \mathrm{m}$ long and 4.5-5.5 $\mu \mathrm{m}$ wide. The transapical costae and keelpunctae are present in equal numbers, the keel-punctae being coarsened continuations of the costae. The number of keel-punctae respective of transapical costae varies between $10-15$ in $10 \mu \mathrm{m}$.

$N$. pungens was found at stations $75,101,130,120,115,135$, and 145 north of the Antarctic Convergence, with a maximum occurring at $41.7^{\circ} \mathrm{S}$ latitude; southward it is found only in small numbers. From other authors it is recorded from localities in the Atlantic and Pacific oceans as far south as $40^{\circ} \mathrm{S}$ latitude (Hasle, 1972).

\section{Nitzschia ritscheri (Hustedt) Hasle (1972) (Plate 3, Figures 1-12)}

Description: Hasle (1965), p. 20-21, pl. 1, fig. 20; pl. 4, fig. 1-7; pl. 7 , fig. 8 .

Remarks: Specimens have dimensions of: length $17-58 \mu \mathrm{m}$, width $6.5-10 \mu \mathrm{m}$. The number of costae is 8-13.5 in $10 \mu \mathrm{m}$ (maximum at 9.3); that of the poroids, 13-23 in $10 \mu \mathrm{m}$ (maximum at 16.5). All transitions between $N$. kerguelensis and $N$. ritscheri were observed.

$N$. ritscheri is an infrequent taxon in samples from the southern Antarctic stations of the Brategg-Expedition (Hasle 1969, 1965). Hustedt (1958) found it in samples from $49^{\circ} \mathrm{S}$ to $69^{\circ} 43^{\prime} \mathrm{S}$ latitude with its greatest abundance at $69^{\circ} 36^{\prime} \mathrm{S}$ latitude. Peragallo (1921) found it near the Argentine Islands near Graham Land. Jousé et al. (1962) and Kozlova (1962) report it in the Indian sector of the Antarctic, and Hargraves (1968) in Antarctic waters and in samples from pack-ice in the Pacific sector of the Antarctic.

It occurred in DSDP Leg 35 plankton samples from stations 140 to 252 (southernmost station at Site 324). Maximum occurrence was found in samples 140-160 (Subantarctic waters) with $11 \%$ abundance of the total assemblage. At the more southern stations percentages of less than $1 \%$ were observed.

\section{Nitzschia separanda (Hustedt) Hasle (1972) \\ (Plate 1, Figures 1-16; Plate 2, Figures 23-29)}

Description: Hasle (1965), p. 26-27; pl. 9, fig. 7-10; pl. 10, fig. 1. Remarks: Observed specimens are 12-45.5 $\mu \mathrm{m}$ long, 5.5-14 $\mu \mathrm{m}$ wide, and have $10-17$ costae in $10 \mu \mathrm{m}$ and $11-20$ poroids in $10 \mu \mathrm{m}$.

It is reported by Hustedt (1958) from Antarctic and Subantarctic zones of the Atlantic Ocean, by Jousé et al. (1962) from the Antarctic region of the Indian Ocean, in considerable numbers by Hasle (1969) from the Antarctic zone in the southeast Pacific, and in somewhat greater quantities by Hargraves (1968) who reported it as well from pack-ice samples. In the DSDP Leg 35 plankton samples, $N$. separan$d a$ was found in greater quantities $(1 \%-11 \%)$ in the Antarctic waters and smaller numbers in the Subantarctic region but nowhere north of station 160 .

\section{Nitzschia seriata Cleve (1883)}

(Plate 3, Figures 19, 20)

Description: Hasle (1965), p. 8-10, pl. 1, fig. 1; pl. 3, fig. 1-7, 10; pl. 4 , fig. 1,2 ; pl. 6 , fig. 2 .

Remarks: This species is characterized by its asymmetric shape, one margin being convex, the other one being approximately straight. The middle part of valves is sometimes elongated and shows more or less parallel margins. Valves taper towards the rounded apices. Keelpunctae and transapical ribs are present in equal numbers, 14-20 in 10 $\mu \mathrm{m}$; the keel-punctae are coarsened continuations of the transapical ribs. Valves are $110-140 \mu \mathrm{m}$ long and 7-8 $\mu \mathrm{m}$ wide.

$N$. seriata seems to be restricted (Hasle 1965, 1972) to the northern part of the northern hemisphere, because records of $N$. seriata (as Pseudonitzschia seriata) from the Antarctic (Helmcke and Krieger, 1962, 1963; Krasske, 1941; and Heiden and Kolbe, 1928) were doubted and related to taxonomic misinterpretation by Hasle (1972). These records are interpreted by both Hasle (1965) and Hustedt (1958) to belong to $N$. fraudulenta and or $N$. pseudoseriata. The latter species is distinguished from $N$. seriata by the symmetry relative to the apical axis.

The frustules found include forms representing the typical seriatatype as well as forms representing the typical pseudoseriata-type, with more or less parallel margins in the middle part of the valves. Differentiation between these two types has not been made. Indeed, their differentiation is questioned.

$N$. seriata was found on Leg 35 north of the Antarctic Convergence at station 101. 
Nitzschia sicula var. bicuneata (Grunow) Hasle, 1960 (Plate 3, Figure 15)

Description: Hasle (1960), p. 26, fig. 16c, d.

Remarks: Only one specimen of this species was found in Subantarctic waters at station 130 . It is $60 \mu \mathrm{m}$ long, $7 \mu \mathrm{m}$ wide, and has 11.5 transapical costae in $10 \mu \mathrm{m}$. Hasle (1964) reports it from Subantarctic and Antarctic zones. Hargraves (1968) found this variety characteristic of the Subantarctic zone.

\section{Nitzschia sicula var. rostrata Hustedt (1958)}

(Plate 3, Figures 13, 14)

Description: Hustedt (1958), p. 180-181, fig. 128-132.

Remarks: The observed specimens are $43-54.5 \mu \mathrm{m}$ long, and 5.5 $\mu \mathrm{m}$ wide. The number of costae in $10 \mu \mathrm{m}$ is $10-11$.

The species was recognized in only two stations 252 and 245 in the Antarctic region. It is described in the literature (Hustedt 1958; Hasle $1960,1964)$ as having a wide distribution mainly in the temperate and subtropical seas, but it is reported, by several authors, from the Antarctic and Subantarctic although in very small numbers (Hustedt, 1958; Hasle, 1969, 1964). Heiden and Kolbe $(1928$, p. 671) record $N$. sicula from pack-ice.

\section{Nitzschia turgidula Hustedt (1958)}

(Plate 4, Figure 4)

Description: Hasle (1965), p. 24-25, pl. 1, fig. 11, pl. 2, fig. 3; pl. 11, fig. 8-13; pl. 12, fig. 1-6.

Remarks: A single specimen was observed (station $175 \mathrm{ca} .60 .5^{\circ} \mathrm{S}$ latitude) measuring $54 \mu \mathrm{m}$ in length and $3 \mu \mathrm{m}$ in width; the number of keel-punctae is 13 in $10 \mu \mathrm{m}$. Transapical costae are not resolvable.

$N$. turgidula was found by Hustedt (1958) in the Subantarctic plankton from $46^{\circ} \mathrm{S}$ to $51^{\circ} \mathrm{S}$ latitude, whereas Hasle (1965) recorded it from $53^{\circ} \mathrm{S}$ to $62^{\circ} \mathrm{S}$ latitude in "Brategg" samples. Hasle found it to be a species belonging to the Subantarctic floral element together with $N$. bicapitata (with a temperature range from $6^{\circ}$ to $2.3^{\circ} \mathrm{C}$ ); south of the Antarctic Convergence it occurs sporadically, probably as an allochthonous element.

\section{Nitzschia turgiduloides Hasle (1965)}

(Plate 4, Figures 1-3)

Description: Hasle (1965), p. 28-29, pl. 12, fig. 9-44; pl. 13, fig. 3-6.

Remarks: The valves of this species have parallel sides and broad rounded apices. The length varies from 100-120 $\mu \mathrm{m}$, width, 2-2.8 $\mu \mathrm{m}$. The keel is eccentric and two keel-punctae in the middle part are more widely spaced than the others. The number of transapical costae in 10 $\mu \mathrm{m}$ is $18-20$; keel-punctae number $11-12$ in $10 \mu \mathrm{m}$.

Only small numbers of this species were found in the region of the Antarctic Convergence. Hasle (1965) reports $N$. turgiduloides in greater abundances from stations near the ice border, thus agreeing with Hargraves' (1968) results.

\section{Nitzschia vanheurckii (M. Peragallo) Hasle (1970)}

(Plate 4, Figure 16)

Description: Hasle (1965), p. 30-31; pl. 12, fig. 13-16; pl. 15, fig. 8.

Remarks: The single recovered specimen is characterized by an enlarged distance between the two middle keel-punctae. Its proportions are: $23 \mu \mathrm{m}$ length, $5 \mu \mathrm{m}$ width; number of costae, 13 in 10 $\mu \mathrm{m}$

It was found at station 235 in Antarctic waters. Sporadic occurrences of the species is reported from Antarctic waters by Peragallo (1921, p. 69); Hustedt (1958, p. 166); Hasle (1969, 1965, p. 31); and Hargraves $(1968$, p. 64$)$. Hasle $(1969,1965)$ found it to be fairly abundant only in samples of the brownish undersurface of shelf ice; she concluded it to have a restricted occurrence and probably a benthonic mode of life.

\section{Genus PLEUROSIGMA W. Smith}

\section{Pleurosigma directum Grunow in Van Heurck (1880)} (Plate 14, Figure 6)

Description: Hendey (1937), p. 348.

Remarks: Specimens vary between $111-172 \mu \mathrm{m}$ in length and 17-30 $\mu \mathrm{m}$ in width; there are 17-30 striae in $10 \mu \mathrm{m}$, the two striation systems crossing each other at an angle of about $60^{\circ}$.
This almost cosmopolitan species is reported from tropical and subtropical areas by such workers as Peragallo (1891, p. 14); Taylor (1966); Simonsen (1974, p. 45); but is found more frequently in the Subantarctic and Antarctic plankton material Karsten (1905); Heiden and Kolbe (1928); Hendey (1937, p. 348); Hustedt (1958, p. 148-149); Manguin (1960, p. 381); Hargraves (1968, p. 82-83); and Hasle (1969, p. 151).

In the present study it was found in small numbers in the Subantarctic waters and the Antarctic Convergence region.

\section{Genus PSEUDOEUNOTIA Grunow in Van Heurck (1880)}

\section{Pseudoeunotia doliolus (Wallich) Grunow in Van Heurck (1880)} (Plate 14, Figure 12)

Description: Hustedt (1959), p. 259-260, fig. 737.

Remarks: Pseudoeunotia doliolus was found in single specimens as far south as $62.5^{\circ} \mathrm{S}$ latitude. Length, $30 \mu \mathrm{m}$; width, $8 \mu \mathrm{m}$; number of transapical costae in $10 \mu \mathrm{m}$ is 12 .

It is one of the most common plankton species in subtropical and tropical areas (Hasle, 1960, p. 21; Simonsen, 1974, p. 56). Single specimens in the plankton of the Subantarctic are reported by Hustedt (1958, p. 158), and Hargraves (1968, p. 85)

\section{Genus RHIZOSOLENIA Ehrenberg (1841)}

\section{Rhizosolenia alata Brightwell (1858)}

(Plate 13, Figure 1)

Description: Hustedt (1930), p. 600-601, fig. 344.

Remarks: A cosmopolitan species found in Antarctic and Subantarctic waters with a maximum of abundance $(10 \%-44 \%)$ of the diatom flora in the Antarctic Convergence zone and just south of it, Rhizosolenia alata was reported from the Antarctic region by Hendey (1937, p. 310); Hustedt (1958, p. 132-133); Manguin (1960, p. 262); Hargraves (1968, p. 85-86); and Hasle (1969, p. 115).

\section{Rhizosolenia alata forma inermis (Castracane) Hustedt (1930)} (Plate 13, Figure 2)

This forma was only found in the region of the Antarctic Convergence whereas Hustedt $(1958$, p. 133) reports a domination of forma inermis in the Antarctic region with the species predominating more in the north. Heiden and Kolbe (1928) observed it exclusively in the pack-ice region.

\section{Rhizosolenia bergonii Peragallo (1892) \\ (Plate 13, Figure 13)}

Description: Hustedt (1930), p. 575-577, fig. 327.

Remarks: The species was found in samples from the Antarctic Convergence and north thereof. Specimens with lateral wing-like basal expansions (Plate 13, Figure 5) were assigned to Rhizosolenia styliformis.

\section{Rhizosolenia hebetata Bailey forma hiemalis Gran (1905)}

(Plate 13, Figures 10-12)

Description: Hustedt (1930), p. 590-592, fig. 337.

Remarks: This forma of Rhizosolenia hebetata has more or less developed lateral wing-like expansions in the "styliformis" manner. It occurs in Antarctic and Subantarctic waters, with predominance in the Antarctic Convergence region.

Rhizosolenia hebetata Bailey forma semispina (Hensen), Gran (1905) (Plate 13, Figures 6, 7)

Description: Hustedt (1930), p. 592, fig. 338

Remarks: A cosmopolitan species observed in Antarctic and Subantarctic waters with a maximum in the region of the Antarctic Convergence. Some specimens have lateral wing-like basal expansions like Rhizosolenia styliformis (Plate 13, Figure 7). Hendey (1937) and Hasle $(1969$, p. 152) point to the difficult distinction between Rhizosolenia hebetata f. semispina and Rhizosolenia styliformis. Most investigators have reported Rhizosolenia hebetata f. semispina from the Antarctic.

\section{Rhizosolenia hebetata forma bidens Heiden (1928)}

(Plate 13, Figure 8)

Remarks: A single specimen with a semispina-like, long spine was recovered at station 190 . 
Rhizosolenia styliformis Brightwell (1858)

(Plate 13, Figures 3-5, 9)

Description: Hustedt (1930), p. 584-588, fig. 333

Remarks: This species is highly variable in morphology and, as already indicated in the description of the other Rhizosolenia species, all transitional forms between the Rhizosolenia species and formae exist.

Most Antarctic workers have reported this cosmopolitan species. In the DSDP Leg 35 plankton material it was found to be in Antarctic as well as in Subantarctic waters but with a maximum abundance in the region of the Antarctic Convergence.

\section{Genus ROPERIA Grunow in Van Heurck (1881)}

\section{Roperia tesselata (Roper) Grunow (1881)}

$$
\text { (Plate 12, Figures 1-14) }
$$

Description: Hustedt (1930), p. 523-524, fig. 297.

Remarks: The valve morphology of this species varies considerably from circular to asymmetrically oval, the latter forms appearing especially in the weakly silicified specimens in the north.

Valve diameter varies from 18-55 $\mu \mathrm{m}$. The submarginal open pseudonodulus is distinctly visible. The number of marginal processes varies from 7 to 15 . Margin is finely striate with $10-22$ striae in $10 \mu \mathrm{m}$. Areolae are uniform or slightly decreasing in size near the margin. The number of loculi in $10 \mu \mathrm{m}$ is $6-11$ and the loculi are arranged in fascicles, with a broad and irregular central area or in straight lines.

Although these multiple variations in shape and structure were separately analyzed, no indication of ecologic importance of any one of them was apparent other than that they all occurred only in the samples north of the Antarctic Convergence. Although this species has mainly a subtropical to tropical distribution (e.g., Simonsen, 1974), it is reported also by Hargraves (1968, p. 91), and Hasle (1969, p. 151) from the Subantarctic region.

\section{Genus SCHIMPERIELLA Karsten (1905)}

\section{Schimperiella antarctica Karsten (1905)}

(Plate 14, Figures 1-5)

Description: Hendey (1937), p. 256.

Remarks: Cells discoid, convex with a broad radially striate margin. The valve diameter is $23-59 \mu \mathrm{m}$, the width of the margin 3-5.5 $\mu \mathrm{m}$. The margin is radially striate with $14-20$ striae in $10 \mu \mathrm{m}$. Areolae are arranged in "eccentrica" manner, fasciculate or grouped to sectors. The central areolae are often somewhat irregularly and loosely grouped; in some specimens a hyaline, irregularly circumscribed central area is present. Areolae decrease in size towards the margin and become more dense; their number in $10 \mu \mathrm{m}$ varies from 8 to 12 . In at least half of the valves a great number (14-19) of fine marginal spines, directed to the center, can be observed. Hendey (1937, p. 256) describes these marginal spines as being typical for the lower valve of this valve-dimorphe species.

Schimperiella antarctica is closely related to Micropodiscus oliveranus (O'Meara) Grunow, which is differentiated from $S$. antarctica by a distinct submarginal process; in addition, in Micropodiscus oliveranus dimorphism in valves is not observed. Much confusion exists concerning these two species; many authorities on Antarctic diatoms report only one of the two species both of which are described as common to Antarctic waters. Hustedt (1959, p. 121); Hasle (1969, p. 151); and Hendey $(1937$, p. 256,258$)$ report only Micropodiscus oliveramus; Hargraves (1968, p. 70) treats them as separate taxa, whereas Jousé et al. (1962, p. 80) and Abbott (1972) treat both species as synonyms. Where, in the studied specimens no submarginal process could be found, such specimens were assigned to Schimperiella antarctica.

The species was found in Antarctic and Subantarctic surface water samples with a maximum abundance in the Antarctic Convergence, and immediately south thereof, where it accounted for $5 \%$ of the diatom flora.

\section{Genus THALASSIONEMA Grunow in Van Heurck (1881)}

\section{Thalassionema nitzschioides Grunow in Van Heurck (1881)} (Plate 14, Figure 11)

Description: Hustedt (1959), p. 244, fig. 725.

Remarks: Specimens recovered from DSDP Leg 35 plankton material are 33-98 $\mu \mathrm{m}$ long, 4-7 $\mu \mathrm{m}$ wide, and have 9-11 marginal punctae in $10 \mu \mathrm{m}$. The valves were variable in outline, from linear with broadly rounded ends to lanceolate with subcapitate ends, heteropolar or isopolar.

A cosmopolitan species (Smayda, 1958, p. 169), it was found in the studied plankton material in small quantities as far south as $63^{\circ} \mathrm{S}$ latitude becoming more common to the north.

Other authors who have reported this species from Subantarctic waters are Heiden and Kolbe (1928, p. 563) and Hustedt (1958, p. 139); Hargraves (1968, p. 96) and Hasle (1969, p. 58) found $T$. nitzschioides even south of the Antarctic Convergence.

\section{Thalassionema nitzschioides var. parva Heiden in Heiden and Kolbe (1928)}

(Plate 14, Figure 10)

Description: Heiden and Kolbe (1928), p. 564, fig. 118.

Remarks: This variety was found only in the northernmost surface-water samples, north of $27.5^{\circ} \mathrm{S}$ latitude.

$$
\text { Genus THALASSIOSIRA Cleve (1873) }
$$

\section{Thalassiosira decipiens (Grunow) Jorgensen (1905)}

(Plate 11, Figures 4-6)

Description: Hustedt (1930), p. 322-323, fig. 158.

Remarks: Valves discoid, slightly convex. The valve diameter is 11$30 \mu \mathrm{m}$, the number of areolae is $9-20$ in $10 \mu \mathrm{m}$. Areolae, largest in the center and decreasing continuously in size towards the margin, are arranged in the same manner as in $T$. eccentrica, but some individuals show a fasciculated structure. The mucilage pore is mostly eccentrical and the marginal spines are strongly developed, varying in number from 4 to 17 .

The species has a wide distribution in temperate and boreal waters in both hemispheres (Hustedt, 1930; Hasle, 1960). It is reported from Antarctic waters by Heiden and Kolbe (1928), Frenguelli and Orlando (1958), Manguin (1960), Cassie (1963), Hargraves (1968), and Abbott (1972). Hendey (1937) found it in the Peru Current off the Chilean coast.

Thalassiosira decipiens was found in DSDP Leg 35 samples only rarely in the Antarctic, but slightly more abundant in the Subantarctic region.

\section{Thalassiosira delicatula Hustedt (1958)}

(Plate 9, Figures 21-25)

Description: Hustedt (1958), p. 110, fig. 8-10.

Remarks: Valves of the observed individuals are convex with a diameter of 8-23 $\mu \mathrm{m}$ and with 7-12 marginal processes. In addition there is always one labiate process, slightly eccentric, and another more or less distinct between the center and the margin. The number of areolae increases toward the margin being 16-20 in $10 \mu \mathrm{m}$ in the inner part of the valve and 22-28 in $10 \mu \mathrm{m}$ near the margin. The species is closely related to Thalassiosira gracilis and is differentiated from the latter species (which has the same geographical distribution) only by its finer structure.

$T$. delicatula is a widespread species in the Southern Ocean (Kozlova, 1962, 1964; Jousé et al., 1962; Hasle and Heimdal, 1970), especially its Antarctic and Subantarctic parts (Hustedt, 1958; Hargraves, 1968; Abbott, 1972). Hasle (1960) reports it from the "Brategg" Expedition as one of the quantitatively important species, at least at certain times.

In the present study it was found in Antarctic waters and in the Antarctic Convergence region.

\section{Thalassiosira eccentrica (Ehrenberg) Cleve (1904)}

(Plate 10, Figures 1, 2, 4, 5)

Description: Hustedt (1930), p. 388, fig. 201 (as Coscinodiscus).

Remarks: In the present investigation Thalassiosira punctifera (Grunow) Fryxell, Simonsen, and Hasle, and most probably $T$. spinosa Simonsen, have been included in $T$. eccentrica because of uncertainties in separating these closely related species. The valves examined measure $25-53 \mu \mathrm{m}$ in diameter and have 6-12 areolae in 10 $\mu \mathrm{m}$. Marginal spines and a labiate process are not always distinct.

The species was found in small numbers in the Antarctic as well as the Subantarctic region. It is a cosmopolitan species (Hustedt, 1930, p. 113; Hasle, 1960, p. 10), but has not been frequently found in high latitudes. It has been reported in small numbers from the Antarctic by Karsten (1905), Van Heurck (1909), Hendey (1937), Heiden and Kolbe (1928), Frenguelli and Orlando (1958), Hustedt (1958), Hargraves (1968), Hasle (1969), and Abbott (1972). 
Thalassiosira eccentrica var. jousei Kanaya in Kanaya and Koizumi (1966)

(Plate 10, Figure 3; Plate 11, Figure 7)

Description: Koizumi (1973), p. 832, pl. 3, fig. 1-6.

Remarks: Individuals of this variation were sporadically found in Antarctic waters and in the Antarctic Convergence. The specimens found are 28-50 $\mu \mathrm{m}$ wide and have 6-8 areolae in $10 \mu \mathrm{m}$. Several small "pores" are clustered in the center.

\section{Thalassiosira gracilis (Karsten) Hustedt (1958)}

(Plate 9, Figures 12-20)

Description: Hustedt (1958), p. 109-110, fig. 4-7.

Remarks: Valves discoid, convex with a diameter of $8-28 \mu \mathrm{m}$. Its most outstanding morphological feature is the widely spaced areolae in the center of the valve with a slightly eccentrically situated mucilage pore. The number of marginal processes is directly related to the diameter of the valve (Figure 15).

This species is frequently reported from the Subantarctic and Antarctic region of the Southern Ocean (Heiden and Kolbe, 1928; Hendey, 1937; Hustedt, 1958; Kozlova, 1962; Hargraves, 1968; Hasle, 1969; and Abbott, 1972).

In the present study it was found in large quantities in samples from the Antarctic waters and the Antarctic Convergence zone, with the same distribution as $T$. delicatula.

\section{Thalassiosira gravida Cleve (1896)}

(Plate 8, Figure 5)

Description: Hustedt (1930), p. 325-326, fig. 161.

Remarks: Valves discoid, flat or slightly convex. Valve diameter 23-37 $\mu \mathrm{m}$. The number of areolae in $10 \mu \mathrm{m}$ is $18-22$, arranged in rows or, in some specimens, in fascicles. Characteristic of the species is a cluster of central pores, a single marginal process, and spines covering the valve surface.

The species is reported from the polar and temperate North Atlantic (Hustedt, 1930, p. 326; Hasle and Heimdal, 1968, p. 366); in this study it was found to be sporadic north of $55^{\circ} \mathrm{S}$ latitude.

\section{Thalassiosira lineata Jousé (1968)}

(Plate 11, Figures 8-10)

Description: Simonsen (1974), p. 9, pl. 1, fig. 6, 7 .

Remarks: This species was found only sporadically north of $52^{\circ} \mathrm{S}$ latitude. Valve diameter is $15-31 \mu \mathrm{m}$, the number of areolae $11-18$ in $10 \mu \mathrm{m}$. Typical are the scattered tubuli over the valve face.

$T$. lineata is described as a tropical to subtropical oceanic species known from the Indian and Pacific oceans (Jousé 1968 and Simonsen, 1974). Results obtained here demonstrate that the species also occurs in colder waters.

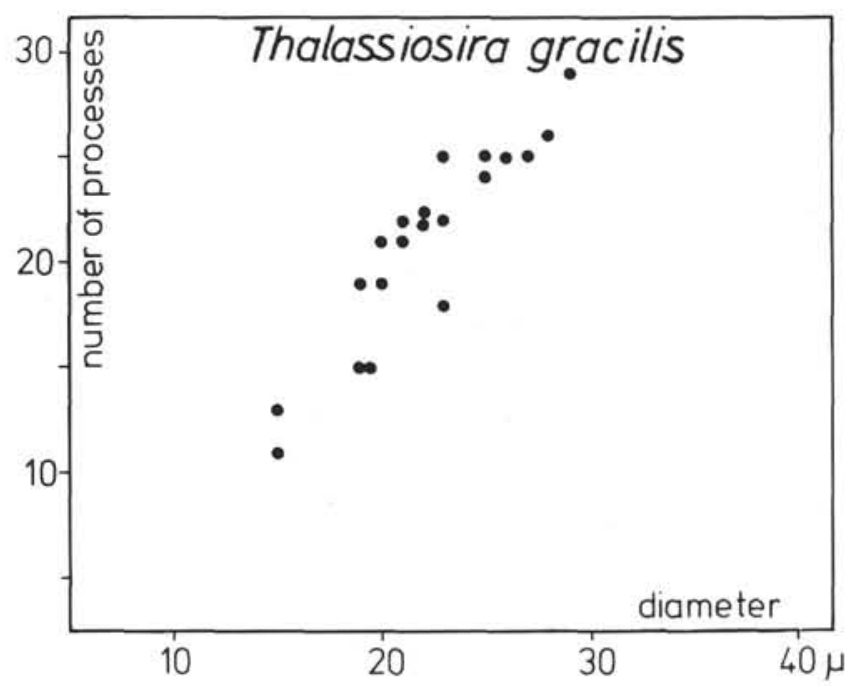

Figure 15. Correlation of number of marginal processes and diameter of valves in Thalassiosira gracilis.
Thalassiosira oestrupii (Ostenfeld) Proshkina-Lavrenko (1955) (Plate 9, Figures 1-11)

Description: Hustedt (1930), p. 318, fig. 155 (as Coscinosira oestrupii).

Remarks: The observed specimens are 10-45 $\mu \mathrm{m}$ wide, and have 610 areolae in $10 \mu \mathrm{m}$. Two types can be distinguished: larger specimens with a nearly flat valve and gradually decreasing areolae size from the center to the margin (Figures 1-5, 11), and smaller convex specimens characterized by large areolae in the center $(6-8$ in $10 \mu \mathrm{m})$ decreasing rapidly in size to the margin (12 in $10 \mu \mathrm{m}$ ) (Figures 6-10); both have the same distribution, occurring in samples from Antarctic and Subantarctic waters with a maximum in the Antarctic Convergence.

The species is more or less cosmopolitan (see locations cited in Hasle, 1960, p. 9) and is reported from the Subantarctic by Hargraves (1968, p. 100), Hasle (1969, p. 152), and Abbott (1972, p. 116).

\section{Thalassiosira poroseriata (Ramsfjell) Hasle ex Hasle and Heimdal (1970) (Plate 9, Figures 26-33)}

Description: Hasle and Heimdal (1970), p. 576, fig. 65-69.

Remarks: Valve discoid, convex, with diameters of $15-32 \mu \mathrm{m}$. The number of areolae is 14-18 in $10 \mu \mathrm{m}$, arranged radially, often fasciculate, and sometimes in an "eccentrica" type. Specific characteristics are: a straight or curved row of 3-5 labiate processes in the center of the valve and two labiate processes grouped together lying between the central group and the margin. In one specimen as many as three labiate processes, forming a line, can be recognized between margin and center. Marginal spines are distinct and varied in number from 9 to 12. A further labiate process can be detected in some specimens in the marginal to submarginal zone.

More heavily silicified and more convex frustules were found in the same samples with identical arrangement of the labiate processes but with a coarser areolation of 8-12 in $10 \mu \mathrm{m}$. The number of central labiate processes never exceeds 4 ; in some specimens only two are present and between the center and the margin there is, in most specimens, only one labiate process; more seldom, even a group of two may be present.

$T$. poroseriata has been found in the Norwegian Sea (Ramsfjell, 1959) and in the northern Antarctic but never inbetween (Hasle and Heimdal, 1970). This concurs with the occurrence of the species in the present material where it was found only north of $52^{\circ} \mathrm{S}$ latitude, between $52^{\circ}$ and $42^{\circ} \mathrm{S}$ latitude, with a maximum at station 130 $\left(50^{\circ} \mathrm{S}\right)$.

\section{Thalassiosira symmetrica Fryxell and Hasle (1972)} (Plate 11, Figures 1-3)

Description: Fryxell and Hasle (1972), p. 312, fig. 37-46.

Remarks: Observed specimens are $25-48 \mu \mathrm{m}$ in diameter and have 9-10 areolae in $10 \mu \mathrm{m}$. In the present material it occurred only sporadically in the Antarctic Convergence and northwards thereof. It is reported by Fryxell and Hasle (1972) and Simonsen (1974) from the tropical and subtropical Pacific and Indian oceans and from one sample of uncertain location from the Shackelton South Pole Expedition in 1908 .

\section{Thalassiosira tumida (Janisch) Hasle (1971) in Hasle, Heimdal, and Fryxell (1971)} (Plate 10, Figures 6, 7)

Description: Hasle, Heimdal and Fryxell (1971), p. 316-318.

Synonym: Coscinodiscus inflatus Karsten.

Remarks: Valve diameter is $52-137 \mu \mathrm{m}$, areolae number $5-12$ in 10 $\mu \mathrm{m}$. The number of marginal labiate processes is 5-9. Larger specimens tend to have a greater number of processes and larger areolae. In the present study the species was found in small numbers in the Antarctic, Subantarctic, and Subtropical waters, which agrees with former records indicating $T$. tumida being a southern circumpolar species with a wide latitudinal range (Manguin, 1966; Cassie, 1963; Balech and El-Sayed, 1965; Hargraves, 1968; Dale, 1968; Hasle, 1969; Kozlova, 1966; Hendey, 1937; Hustedt, 1958).

Thalassiosira sp. a

(Plate 8, Figures 15-17)

The valve is convex, discoid, with valve diameter ranging between 23-32 $\mu \mathrm{m}$. The number of areolae in $10 \mu \mathrm{m}$ is $10-15$, and are grouped 
in radial rows, decreasing in size from the center to the margin. In the center they are irregularly and loosely arranged. The number of marginal spines increases with the size of the diameter and varies from 16 to 29 .

It was found in quantities of $0.5 \%-1 \%$ of the diatom flora in the samples from the Antarctic region and the Antarctic Convergence.

Thalassiosira sp. b

(Plate 8, Figure 12-14)

Thalassiosira sp.

(Plate 8, Figures 10, 11)

Thalassiosira sp. d

(Plate 8, Figures 8, 9)

Thalassiosira sp. e

(Plate 8, Figures 6, 7)

Thalassiosira sp. $f$

(Plate 8, Figures 1, 2)

Thalassiosira sp. $\mathrm{f}$ is discoid and flat, the valve diameter ranging from 32 to $43 \mu \mathrm{m}$. The areolae form radial rows grouped together into fascicles. One areolae row extends from the center to the margin; shorter neighboring rows on both sides are parallel to the first. The areolae in the center form an irregular group mostly surrounded by a rim. The size of the areolae decrease from the center (9-10 areolae in $10 \mu \mathrm{m})$ to the margin $(12-13$ areolae in $10 \mu \mathrm{m})$. Marginal spines number from 18-20 with one process lying submarginal and outside of this ring.

It was found in samples from Antarctic and Subantarctic waters, with a maximum abundance ( $2 \%$ of the diatom flora) south of the Antarctic Convergence.

Thalassiosira sp. $\mathrm{g}$

(Plate 8, Figures 3, 4)

\section{Genus THALASSIOTHRIX Cleve and Grunow (1860)}

\section{Thalassiothrix longissima Cleve and Grunow (1880) (No illustration)}

Description: Hustedt (1959), p. 247, fig. 726.

Remarks: Although Thalassiothrix antarctica Schimper, is differentiated from Thalassiothrix longissima by the lack of marginal spines, there is a continuous transition between the two forms in the present material and they have been included in the one species $T$. longissima. Cupp (1943, p. 184) also found these "connecting forms", Heiden and Kolbe (1928, p. 565) and Hendey (1937, p. 336) treat them as synonyms.

Thalassiothrix longissima is one of the most common plankton species of the oceans. It is reported from the Antarctic and Subantarctic region by Heiden and Kolbe (1928, p. 565), Hendey (1937, p. 335); Hustedt (1958, p. 141-142); Manguin (1960, p. 301); Hargraves (1968, p. 101-104); Hasle (1969, p. 152); and Abbott (1972, p. 117).

In the present study the species was found in nearly all samples from Antarctic and Subantarctic surface water samples in percentages varying from $0.2 \%$ to $13 \%$ of the diatom flora.

\section{Genus TRACHYNEIS Cleve (1894)}

\section{Trachyneis aspera (Ehrenberg) Cleve (1894)}

$$
\text { (Plate 14, Figure 13) }
$$

Description: Cleve (1894), v. 26, no. 2, p. 191.

Remarks: This cosmopolitan species was found only sporadically at station $101\left(41.8^{\circ} \mathrm{S}\right.$ latitude).

\section{Genus TROPIDONEIS Cleve (1891)}

\section{Tropidoneis antarctica (Grunow) Cleve (1894)}

$$
\text { (Plate 14, Figure 8) }
$$

Description: Hustedt (1958), p. 149-150.

Remarks: The observed specimens are 39-77 $\mu \mathrm{m}$ long, 13-18 $\mu \mathrm{m}$ wide, and the number of transapical striae is $18-22$ in $10 \mu \mathrm{m}$, not becoming denser towards the ends. The transapical striae are crossed by finer apical striae, numbering $21-23$ in $10 \mu \mathrm{m}$.

The species is reported to be common in the plankton of the Antarctic by Hustedt (1958, p. 149); Manguin (1960, p. 321); and Hasle (1969, p. 152). It was found in the examined plankton material in Antarctic and Subantarctic surface water samples with a maximum frequency ( $1.6 \%$ of the diatom flora) in the Antarctic Convergence zone.

\section{REFERENCES}

Abbott, W.H., 1972. Vertical and lateral patterns of diatomaceous ooze found between Australia and Antarctica: Ph.D. Thesis Department Geol., University of South Carolina.

1974. Observations on Pleistocene diatoms from the southeast Indian Ocean: Symp. Marine Plankt. and Sediments, Kiel, Abstracts, p. 4. in press. Temporal and spatial distribution of Pleistocene diatoms from the southeast Indian Ocean: Nova Hedw.

Balech, E. and El-Sayed, S.Z., 1965. Microplankton of the Weddell Sea. Biol. Antarct., Seas, II: Antarctic Res. Ser., v. 5 , p. $107-124$.

Berger, W.H. and Parker, F.L., 1970. Diversity of planktonic foraminifera in deep-sea sediments: Science, v. 168, p. 1345-1347.

Boden, B.P., 1949. The diatoms collected by the U.S.S. Cacopan in the Antarctic in 1947: Sears Found. J. Mar. Res., v. 8, p. 6-13.

Bory de Saint Vincent, J. Baptiste Marcellin, 1822-1831. Collaborator in "Dictionaire classique d'Histoire Naturelle": 17 volumes, Paris.

Botnikov, V.N., 1964. Geographic position of the Antarctic Convergence Zone in the Pacific Ocean: Soviet Antarctic Exped. Inform. Bull., v. 4, p. 324-327 (English translation).

Boyer, C.S., 1927. Synopsis of North American Diatomaceae: Acad. Nat. Sci. Philadelphia Proc., v. 78, suppl., part 1, p. 1-228; 79, suppl., part 2, p. 229-583.

Brightwell, T., 1858. Remarks o, the genus "Rhizosolenia" of Ehrenberg: Quart J. Micr. Sii., v. 6, p. 93.

1860. On some of the rarer or undescribed species of Diatomaceae: Quart. J. Micr. Sci., v. 7, p. 179-181.

Bunt, J.S., 1961. Introductory studies: Hydrology and plankton, Mawson: ANARE Rept. Ser., B 3, p. 1-135.

Burckle, L.H., 1974. The distribution of displaced Antarctic diatoms in the Argentine Basin (abstract): Symp. Marine Plankt. and Sediments, Kiel, Abstracts, p. 14.

Burkholder, P.R. and Sieburth, J.M., 1961. Phytoplankton and chlorophyll in the Gerlache and Bransfield Straits of Antarctica: Limnol. Oceanogr., v. 6, p. 45-52.

Cassie, V., 1963. Distribution of surface phytoplankton between New Zealand and Antarctic, December 1957: T.A.E. Sci. Rept., v. 7, p. 1-9.

Castracane, C.A.F.A., 1886. Report on the scientific results of the voyage of H.M.S. Challenger during the years 18731876: Botany, v. 2, p. 1-178.

Cleve, P.T., 1881. Diatomées rares ou nouvelles: Le Diatomiste, v. 1, p. 53.

1883. Diatoms collected during the expedition of the Vega: Vega Exped. Jakttagelser, v. 3, p. 455-517.

1894-1895. Synopsis of the naviculoid diatoms: Kongliga Svenska Vetenskaps Akad. Handlg., v. 26, p. 1194; v. 27, p. 1-219 (Part I, 1894, part II, 1895).

1896. Diatoms from Baffin Bay and Davies Strait: Bih. Svenska Vetenskaps Akad. Handlg., v. 22, p. 1-22. 1897. A treatise on the phytoplankton of the Atlantic and its tributaries and on the periodic changes of the plankton of the Skagerak: Uppsala, $27 \mathrm{p}$. 
1897. Report on the phytoplankton collected on the expedition of H.M.S. "Research" 1896. Part 3, Scientific investigations: Rept. Fishery Bd. Scott., v. 15, p. $297-$ 304.

1900. Plankton from the southern Atlantic and the southern Indian Ocean: Ofversigt af Kongliga Vetenskaps. Akad. Förhandlingar, v. 57, p. 919-1038.

1904. Plankton tables for the North Sea: Cons. Explor. Mer. Bull., 1903-1904, p. 216.

Cleve, P.T. and Grunow. A., 1880. Beiträge zur Kenntnis der arktischen Diatomeen: Kongl. Svenska Vetenskaps-Akad. Handlingar, v. 17, p. 1-121.

Cleve, P.T. and M $\phi$ ller, J.D., 1882. Diatoms: 6th part, no. 277-324, Uppsala, p. 1-6.

Cupp, E.E., 1943. Marine plankton diatoms of the west coast of North America Scripps Inst. Oceanogr. Bull., Tech. Ser., v. 5 .

Dale, R.L., 1968. International Weddell Sea Oceanographic Expedition 1968: Antarctic J. U.S., v. 3, p. 80-84.

David, P.M., 1958. The diatribution of the Chaetognatha of the Southern Ocean: Discovery Rept., v. 29, p. 199-228.

Deacon, G.E.R., 1937. The hydrology of the Southern Ocean: Discovery Rept., v. 15, p. 1-24.

1963. The Southern Ocean, ideas and observations on progress in the study of seas. In Hill, M.N. (Ed.), The sea, vol. 2: New York (Interscience), p. 281-296.

Donahue, J.G., 1970a. Diatoms as quaternary biostratigraphic and plaeoclimatic indicators in high latitudes of the Pacific Ocean: Ph.D. Thesis, Faculty of Pure Science. Columbia University.

1970b. Pleistocene diatoms as climatic indicators in North Pacific sediments: Geol. Soc. Am. Mem. 126, p. 121-138.

Ehrenberg, C.G., 1830 (1832). Beiträge zur Kenntnis der Organisation der Infusorien und ihrer geographischen Verbreitung besonders in Sibirien: Abh. Kgl. Akad. Wiss. Berlin, p. 1-88.

1837. Die fossilen Infusorien und die lebendige Dammerde: Preuss. Physik. Abh. Kgl. Akad. Wiss. Berlin. 1838. Die Infusionstierchen als vollkommene Organismen. Ein Blick in das tiefere organische Leben der Natur: Leipzig (Leopold Voss), p. 1-7, 1-548.

1839. Uber noch jetzt zahlreich lebende Tierarten der Kreidebildung und den Organismus der Polythalamien: Abh. Kgl. Akad. Wiss. Berlin, p. 81-174.

1844. Einige vorläufige Resultate der Untersuchungen der von der Sudpolreise des Capitain Ross, so wie von den Herren Schayer and Darwin zugekommen Materialien: Monatsber. Preuss. Akad. Wiss., p. 182-207. 1844 (1845). Uber zwei neue Lager von Gebirgsmassen aus Infusorien als Meeresabsatz in Nord-Amerika und ein Vergleich derselben mit den organischen Kreidegebilden in Europa und Afrika: Abh. Kgl. Akad. Wiss. Berlin, p. 57-97.

El-Sayed, S.Z., 1966a. Biologie der Antarktischen Meere: Umschau, v. 8, p. 1-7.

1966b. Phytoplankton production in Antarctic and Subantarctic waters (Atlantic and Pacific sectors): 2nd Int. Oceanogr. Congr. Moscow 1966, p. 106-107.

El-Sayed, S.Z. and Mandelli, E.F., 1965. Primary production and standing crop of phytoplankton in the Weddell Sea and Drake Passage: Biology of the Antarctic Seas II: Antarctic Res. Ser., v. 5, p. 87-106.

Frenguelli, J., 1938. Diatomeas de la Bahia de San Blas (Provinca de Buenos Aires): Rev. Mus. de la Plata, v. 1, p. 251-337.

1960. Diatomeas y Silicoflagelados recogidas en Tierra Adelia durante la Expediciones Polare Francesas de Paul-Emile Victor (1950-1952): Rev. Algol. N.S., v. 5, p. 3-48.
Frenguelli, J. and Orlando, H., 1958. Diatomeas y silicoflagelados del sector Antarctico Sudamericano: Inst. Antarctico Argentino, v. 5, p. 1-191.

1959. Operacion Merluza. Diatomeas y silicoflagelados del plankton del "VI Crucero": Serv. Hidrogr. Naval., Publ. H. 619, p. 1-62.

Fritsch, F.E., 1912. Freshwater algae: Nat. Antarctic Exped. 1901-1904. Nat. Hist., v. 6, p. 1-60.

Fryxell, G.A. and Hasle, R.G. (1973). Coscinodiscineae: Some consistent patterns in diatom morphology: Nova Hedw., Beihft., v. 45, p. 69-98.

Fukase, S., 1962. Oceanographic condition of surface water between the south end of Africa and Antarctica: Antarctic Rec., v. 15, p. 53-110.

Fukase, S. and El-Sayed, S.Z., 1965. Studies on diatoms of the Argentine Coast, the Drake Passage and the Bransfield Strait: Oceanogr. Mag., v. 17, p. 1-11.

Gombos, A., 1974. Diatoms as indicators of Antarctic interglacials (abstract): Symp. Marine Plankt. and Sediment, Kiel, Abstracts, p. 27.

Gordon, A., 1967. Structure of Antarctic waters between $20^{\circ}$ west and $170^{\circ}$ west: Antarctic Map Folio Series, Folio 6, American Geographical Society, p. 1-10.

1971a. Oceanography of Antarctic Waters. In Reid, J.L. (Ed.), Antarctic Oceanology I, Antarctic Research Series: v. 15, p. 196-203.

1971b. Recent physical oceanographic studies of Antarctic Waters: Res. in the Antarctic, no. 93, p. 609 629.

1971c. Spreading of Antarctic bottom Waters II. In Gordon, A.L. (Ed.), Studies in physical oceanography. A tribute to Georg Wüst on his 80th birthday: New York (Gordon and Breach).

Gran, H., 1905. Diatomeen. In Brandt, K. and Apstein, C. (Eds.), Nordische Plankton: v. 19, p. 1-146.

Gray, S.F., 1821. Diatomaceae: London (Balduin, Cradock \& Joy), v. 1, p. 293-295.

Greville, R., 1859. Descriptions of diatomaceae observed in California guano: Quat. J. Micr. Sci., v. 7, p. 155-166.

Grunow, A., 1867. Diatomeen auf Sargassum von Honduras: Hedwigia, v. 6, p. 17-37.

1877. New diatoms from Honduras: Mon. Micr. J., v. 18 , p. $165-186$. Taf. 118 .

1881. In Van Heurck, Synopsis....., Erklärung zu

1884. Die Diatomeen von Franz-Josephs Land: Denkschrift. Math. Naturw. Klasse kaiserl. Akad. Wiss., v. 48, p. $53-112$.

Hargraves, P., 1968. Species composition and distribution of net plankton diatoms in the Pacific sector of the Antarctic Ocean: Ph.D. Thesis, Columbia University.

Hart, T.J., 1934. On the phytoplankton of the southwest Atlantic and the Bellingshausen Sea, 1929-1931. Discovery Rept., v. 8, p. 1-268.

1942. Phytoplankton periodicity in Antarctic surface waters: Discovery Rept., v. 21, p. 263-348.

Hasle, G.R., 1960. Phytoplankton and ciliate species from the tropical Pacific: Skrift. det Norske Videnskaps-Akad., Oslo, Matemat. Naturv., Kl, no. 2, p. 1-50.

G.R., 1964. Nitzschia and Fragilariopsis species studied in the light and electron microscopes. I. Some marine species of the groups Nitzschiella and Lanceolatae: Skr. Norske Vidensk.

1965. Nitzschia and Fragilariopsis species studied in the light and electron microscopes. III. The genus Fragilariopsis: Skr. norske Vidensk.-Akad. 1. Mat.-Nat. Kl., v. 21, p. 1-49.

1969. An analysis of the phytoplankton of the Pacific Southern Ocean: abundance, composition, and distribution during the Brategg Expedition, 1947-1948. 
Hvalradets Skrifter, Norske Videskaps-Akademi I Oslo, no. 52 , p. 1-168.

1972. Fragilariopsis Hustedt as a section of the genus Nitzschia Hassal: Nova Hedw., Beihft., v. 39, p. 111-119.

Hasle, G.R. and Heimdal, B.R., 1967. Morphology and distribution of the marine centric diatom Thalassiosira antarctica. J. Roy. Micr. Soc., v. 88, p. 357-369.

1968. Morphology and distribution of the marine centric diatom Thalassiosira antarctica COMBER: J. Roy. Micr. Soc., v. 88 , p. 357-369.

1970. Some species of the centric diatom genus Thalassiosira studied in light and electron microscopes: Nova Hedw., Beihft., v. 31, p. 559-581.

Hasle, G.R., Heimdal, B.R., and Fryxell, G.A., 1971. Morphologic variability in fasciculated diatoms as exemplified by Thalassiosira tumida (Janisch) Hasle, comb. nov.: Ant. Res. Ser. 17 (Biol. Antarctic Seas IV), p. 313333.

Heiden, H. and Kolbe, R.W., 1928. Die Marinen Diatomeen der Deutschen Sudpolar Expedition 1901-1903: Deutsche Südpolar Exped., v. 8, p. 450-714.

Helmcke, J.-G. and Krieger, W., 1962/1963. Diatomeenschalen im elektronenmikroskopischen Bild: II and IV, Weinheim.

Hendey, N.I., 1937. The plankton diatoms of the Southern Ocean: Discovery Rept., v. 16, p. 151-364.

Husted, F., 1930. Die Kieselalgen Deutschlands, Osterreichs und der Schweiz: In L. Rabenhorst, Kryptogamen-Flora von Deutschland, Osterreich und der Schweiz, v. 7, pt. 1. 1952. Diatomeen aus der Lebensgemeinschaft des Buckelwals (Megaptera nodosa Bonn.): Arch. Hydrobiol., v. 46, p. 286-298.

, 1958. Diatomeen aus der Antarktis und dem Südatlantik: Deutsche Antarkt. Exped. 1938-1939, v. 2, p. 103-191.

1959. Die Kieselalgen Deutschlands, Osterreichs und der Schweiz unter Berücksichtigung der übrigen Länder Europas sowie der angrenzenden Meeresgebiete: Kryptogamen-Flora von Deutschl., Osterr. u.d. Schweiz, Teil 2, v. 7, p. 1 .

Janisch, C., 1878. In A. Schmidt, Atlas der Diatomaceenkunde, Taf. 57.

1891. Diatomeen der Gazelle-Expedition. 18741876: Tafelerklärungen von $\mathrm{O}$. Witt, unpublished.

Jörgensen, E., 1905. The protist plankton and the diatoms in bottom samples. O. Nordgaard, Hydrographical and biological investigations in Norwegian Fjords, Bergens Museum Skrift., Bergen.

John, G.R., 1936. The southern species of the genus Euphausia. Discovery Rept., v. 14, p. 193-324.

Jousé, A.P., 1968. Species novae bacillariophytorum in sedimentis fundi oceani Pacifici et Mario Ochotensis inventae. Novitates Systematicae Plantarum non vascularum: Akad. Sci. SSSR, Inst. Botanicum nomine V.L. Komarovii, p. 12-21.

Jousé, A.P., Koroleva, G.S., and Nagaeva, G.A., 1962. Diatomorrje Vodorosli v poverkhnostnom Sloe Donnykh Osadkov Indiiskogo Sketora Antarktiki: Trudy Inst. Oceanol. Akad. NAUK, SSSR, v. 61, p. 19-92 (in Russian).

Kanaya, T. and Koizumi, I., 1966. Interpretation of diatom thanatocoenoses from the North Pacific applied to a study of core V20-130 (Studies of deep-sea core V20-130, Part IV): Sci. Rept., Tohoku Univ., Japan, 2nd. ser. (geol.), v. 37, p. $89-130$.

Karsten, G., 1905. Das Phytoplankton des Antarktischen Meeres. In Chun, C. (Ed.), Wissenschaftliche Ergebnisse der Deutschen Tiefsee-Expedition auf dem Dampfer Valdivia, 1898-1899: Jena, p. 1-136.
Koizumi, I., 1966. Tertiary stratigraphy and diatom floras of the Ajigassawa District, Aomori Prefecture, Northeast Japan Tohoku Univ., Inst. Geol. Paleontol., Contrib. no., 62 (in Japanese with English abstract).

1973. The late Cenozoic diatoms of Sites 183-193, Leg 19, Deep Sea Drilling Project. In Creager, J.S., Scholl, D.W., et al., Initial Reports of the Deep Sea Drilling Project, Volume 19: Washington (U.S. Government Printing Office), p. 805-856.

Kolbe, R.W., 1954. Diatoms from equatorial Pacific cores: Rept. Swedish Deep-Sea Exped., v. 6, p. 1-48.

Kort, V.G., 1964. Antarctic Oceanography. In Odishaw, H. (Ed.), Research in Geophysics: v. 2, p. 309-333.

Kozlova, O.G., 1962. Specific composition of diatoms in the waters of the Indian sector of the Antarctic: Trud. Inst. Oceanol., v. 61, p. 3-18 (in Russian with English summary).

1964. Diatoms of the Indian and Pacific sectors of the Antarctic: Acad. Sci. SSSR, Inst. Oceanol. (Translated in 1966 by the Israel Program for Scientific translations, U.S. clearing house for Federal Scientific and Technical Information, TT 66-51154), p. 1-191.

Krasske, G., 1941. Die Kieselalgen des chilenischen Küstenplanktons: Archiv Hydrobiol., v. 38, p. 275.

Kützing, F.T., 1833. Synopsis Diatomacearum oder Versuch einer systematischen Zusammenstllung der Diatomeen: Linnaea, v. 8, p. 529.

Lauder, H.S., 1864. On new diatoms: Trans. Micr. Soc. London, v. 12 , p. 6-8.

Mackintosh, N.A., 1946. The Antarctic Convergence and the distribution of surface temperatures in Antarctic Waters: Discovery Rept., v. 23, p. 177-180.

1964. Distribution of the plankton in relation to the Antarctic Convergence: Roy. Soc. London Proc., v. 281 , p. $21-38$.

1960. The pattern of distribution of Antarctic fauna: Roy. Soc. London Proc., v. 152, p. 624.

Mandelli, E.F. and Burkholder, P.R., 1966. Primary productivity in the Gerlache and Bransfield Straits of Antarctica: Sears Found. J. Mar. R5s., v. 24, p. 15-27.

Manguin, E., 1957. Premier inventaire de Diatomées de la Terre Adélie. Antarctic espèces nouvelles: Rev. Algol., v. 3, p. 111-134.

1960. Les diatomees de la Terre Adelie: Ann. Sci. Naturelles, v. 1, p. 223-385.

Mann, A., 1973. Diatoms: Sci. Rept. Austral. Antarctic Exped., v. 1, p. 1-82.

McCollum, D.W., 1975. Leg 28 Diatoms. In Hayes, D., Frakes, L.A., et al., Initial Reports of the Deep Sea Drilling Project, Volume 28: Washington (U.S. Government Printing Office), p. 515-572.

Ostapoff, F., 1962. The salinity distribution at 200 meters and the Antarctic frontal zones: Deut. Hydrograph. Z., v. 15, p. 133-142.

Oxford Atlas. 1963, London.

Peragallo, H., 1892. Monographie du genre Rhizosolenia: Le Diatomiste, v. 1, p. 99-117.

Peragallo, M., 1921. Diatomees d'eau douce, et Diatomees d'eau salée: Deux. Exped. Antarc. Franc. 1908-1910, Docum. Sci., Bot., v. 16, Paris.

Petit, P. 1889. Classification. In Pelletan, J. (Ed.), Les diatomées: Paris (Baillère), p. 189-208.

Proshkina-Lavrenko, A.J., 1955. Plankton diatoms of the Black Sea): Moscow-Leningrad (in Russian).

Ralfs, J., 1861. In Pritchard. A History of infusoria, living and fossil: Ed. IV, revised and enlarged, London.

Ramsfjell, E., 1959. Two new phytoplankton species from the Norwegian Sea, the diatom Coscinodiscus poroseriata, and the dinoflagellate Goniaulax parva: Nytt. Mag. Bot., v. 7, p. 175-177. 
Schmidt, A., et al., 1874. Atlas der Diatomaceenkunde. Heft 1-120, Tafeln 1-460 (Tafeln 1-216 A. Schmidt; 213-216 M. Schmidt; 217-240 (1900-1901) F. Fricke; 241-244 (1903) H. Heiden; 245-246 (1904) O. Müller; 247-256 (1904-1905) F. Fricke; 257-264 (1905-1906) H. Heiden; 265-268 (1906) F. Fricke; 269-472 (1911-1959) F. Hustedt): Leipzig (R. Reisland).

Schrader, H.-J., 1969. Die pennaten Diatomeen aus dem Obereozän von Oamaru, Neuseeland: Nova Hedw., Beihft., v. 28, p. 1-124.

1974. Proposal for a standardized method of cleaning diatom-bearing deep-sea and land-exposed marine sediments: Nova Hedw., Beihft., v. 45, p. 403-409.

Shadbolt, G., 1854 (1853). A short description of some new forms of Diatomaceae from PortNatal: Micrc. Soc. London Trans., v. 2, p. 13-18.

Simonsen, R., 1972. Uber die Diatomeengattung Hemidiscus WALLICH und andere Angehörige der sogenannten Hemidiscaceae: Veröfftl. Inst. Meeresforschung Bremerhaven, v. 13 , p. $265-273$.

1974. The diatom plankton of the Indian Ocean Expedition of R.V. "Metero" 1964-1965: "Meteor"Forschungsergb., Reihe D, No. 19, 66 p.
Taylor, F.J.R., 1966. Phytoplankton of the south-western Indian Ocean: Nova Hedw., v. 12, p. 433-476.

Van Heurck, H., 1909. Diatomees. Resultats du voyage du S.Y. "Belgica" en 1897-1899: Rapports Scientifiques, Botaniques, p. 3-126.

Wallich, G.C., 1860. On the siliceous organisms found in the digestive cavities of the Salpae, and their relation to the Flint nodules of the Chalk Formation: Micr. Soc. Trans. London, v. 8, p. 36.

Werff, van der A., 1954. Diatoms in plankton samples of the Willem Barendsz-Expedition 1947: Hydrobiologia, v. 6, p. 331-333.

Wexler, H., 1959. The Antarctic convergence or divergence? In atmosphere and the sea in motion. In Bolin, B. (Ed.), New York (Rockefeller Inst.), p. 107-120.

Wood, E.J., 1965. Antarctic phytoplankton studies: Proc. Linn. Soc. N.S.W., v. 85, p. 215-229.

Wyrtki, K., 1960. The Antarctic Circumpolar Current and the Antarctic Polar Front: Dtsch. Hydrogr. Z., v. 13, p. 153174. 



\section{PLATE 1}

(Magnification $1500 \times$ )

Figures 1-16 Nitzschia separanda (Hustedt) Hasle.

1. Station 280.

2. Station 300 .

3. Station 300 .

4. Station 305.

5. Station 307.

6. Station 307.

7. Station 307.

8. Station 300 .

9. Station 300 .

10. Station 252 .

11. Station 240.

12. Station 252.

13. Station 252.

14. Station 305.

15. Station 300.

16. Station 252.

Figures 17-39 Nitzschia angulata (O'Meara) Hasle.

17. Station 307.

18. Station 307.

19. Station 307.

20. Station 252.

21. Station 307.

22. Station 252.

23. Station 252.

24. Station 252.

25. Station 252.

26. Station 190.

27. Station 235.

28. Station 252.

29. Station 235 .

30. Station 165.

31. Station 307.

32. Station 252.

33. Station 305.

34. Station 225.

35. Station 252.

36. Station 252.

37. Station 201.

38. Station 305.

39. Station 305. 
PLATE 1

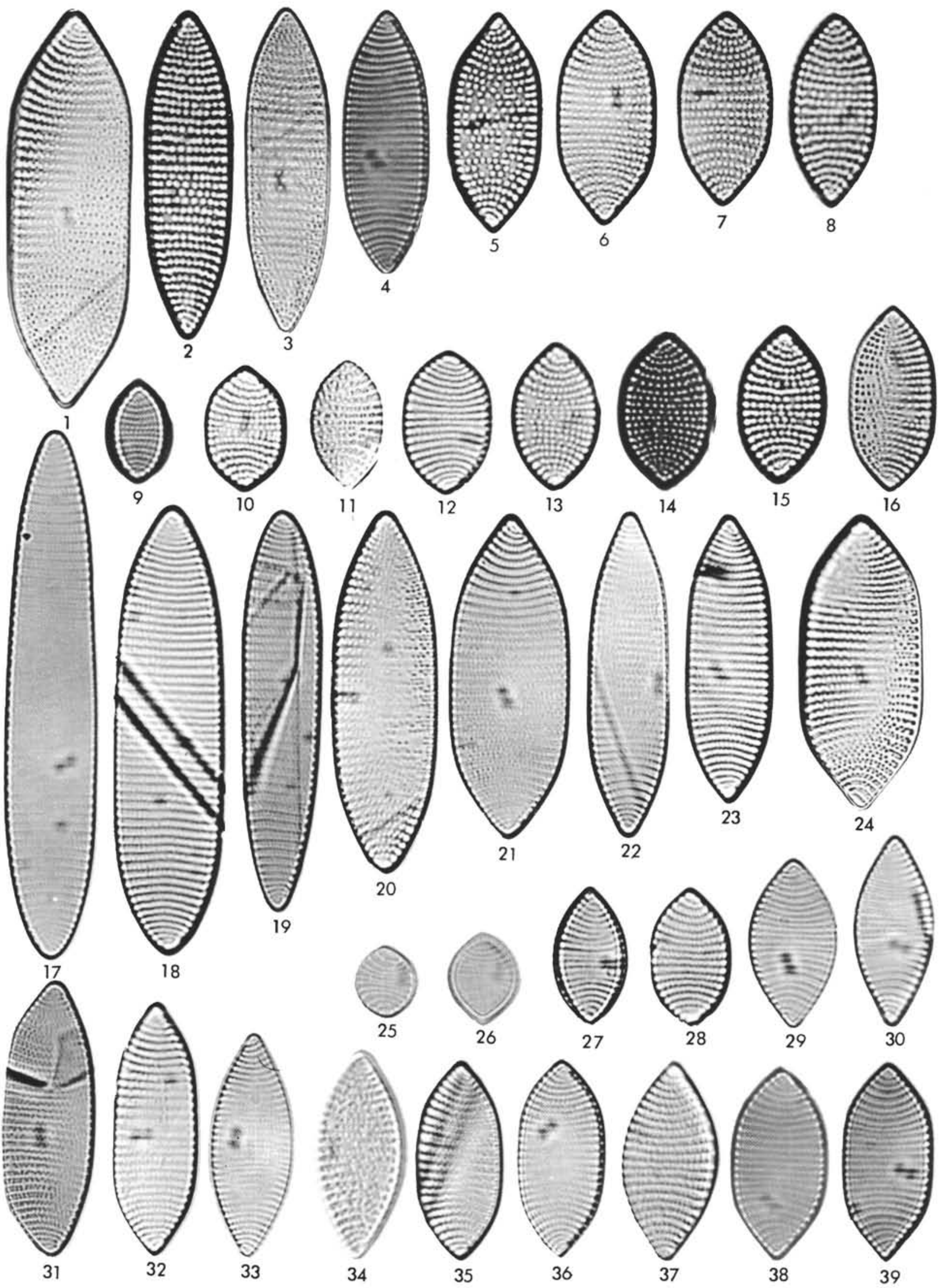




\section{PLATE 2}

(Magnification $1500 \times$ )

Figures 1-5 Nitzschia grunowii (Cleve) Hasle.
1. Station 101.
2. Station 140 .
3. Station 140 .
4. Station 140.
5. Station 145.

Figures 6-11 Nitzschia pseudonana Hasle.
6. Station 145.
7. Station 140.
8. Station 230.
9. Station 175.
10. Station 145.
11a. Station 270 .
11b. Station 145.

Figures 12-14 Nitzschia peragallii Hasle.

12. Station 245.

13. Station 252.

14. Station 265 .

Figures 15-18 Nitzschia obliquecostata (Van Heurck) Hasle.

15. Station 140.

16. Station 140.

17. Station 195.

18. Station 252.

Figures 19-30 Nitzschia kerguelensis (O'Meara) Hasle.

19. Station 265.

20. Station 240.

21. Station 240.

22. Station 270.

23. Station 235 .

24. Station 180.

25. Station 185 .

26. Station 240.

27. Station 265.

28. Station 240.

29. Station 300 .

30. Station 240 . 
PLATE 2

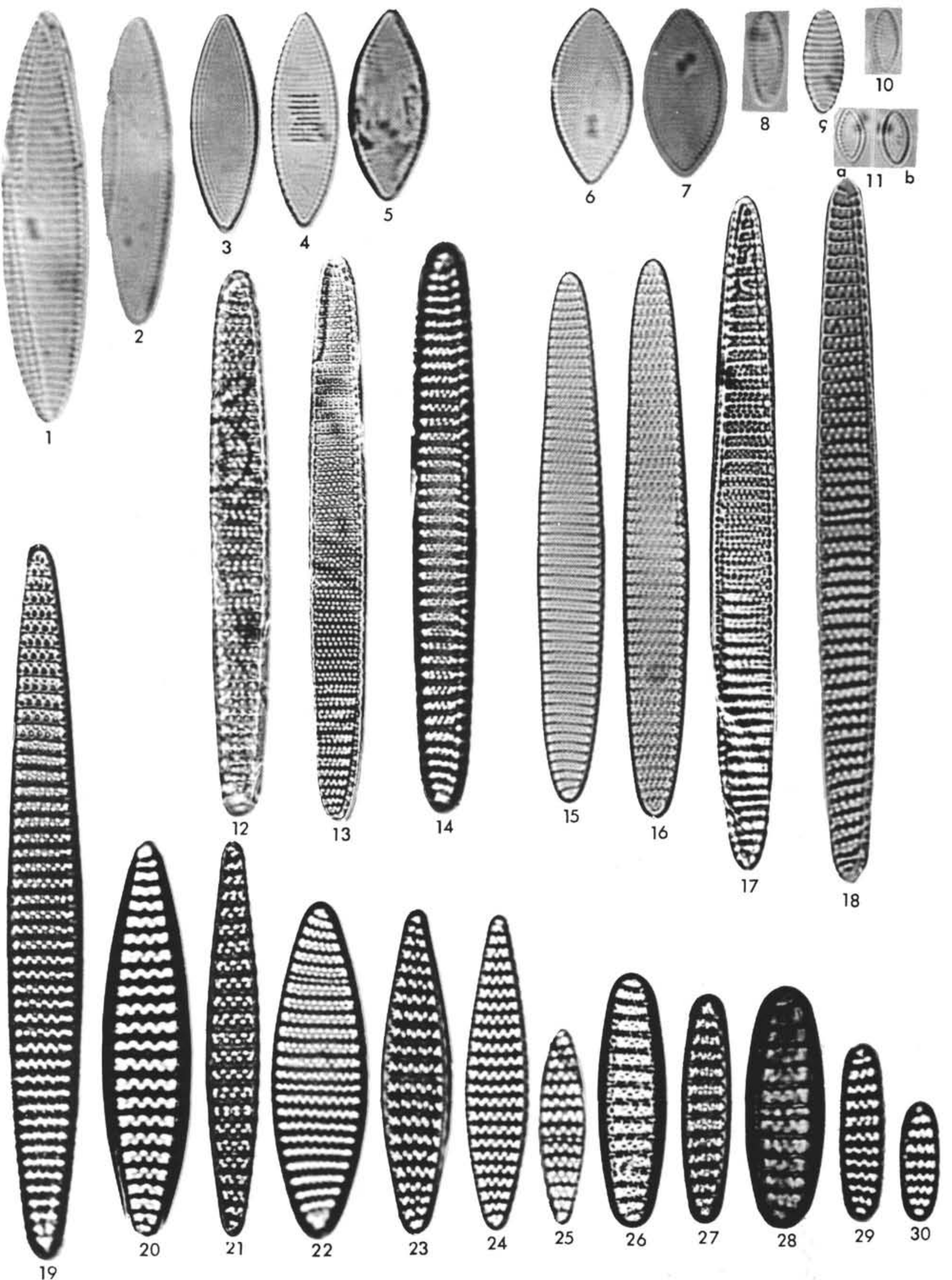


PLATE 3

(Magnification $1500 \times$ )

Figures 1-12 Nitzschia ritscheri (Hustedt) Hasle.

1. Station 140.

2. Station 252 .

3. Station 145 .

4. Station 140.

5. Station 230 .

6. Station 140 .

7. Station 140 .

8. Station 205.

9. Station 175.

10. Station 300.

11. Station 140.

12. Station 155 .

Figures 13, 14 Nitzschia sicula var. rostrata Hustedt.

13. Station 245.

14. Station 252.

Figure $15 \quad$ Nitzschia sicula var. bicuneata (Grunow) Hasle.

Figure $16 \quad$ Nitzschia kolaczeckii Grunow. Station 140.

Figure $17 \quad$ Nitzschia fraudulenta Cleve. Station 101.

Figure $18 \quad$ Nitzschia pungens Grunow. Station 101.

Figures 19,20. Nitzschia seriata Cleve. Station 101.

Figures 21-23. Nitzschia heimii Manguin. Station 201.

Figures 24-26. Nitzschia lineola Cleve.

24. Station 205.

25. Station 201.

26. Station 185 .

Figures 27-29 Nitzschia bicapitata Cleve.

27. Station 165.

28. Station 190.

29. Station 170 . 
PLATE 3

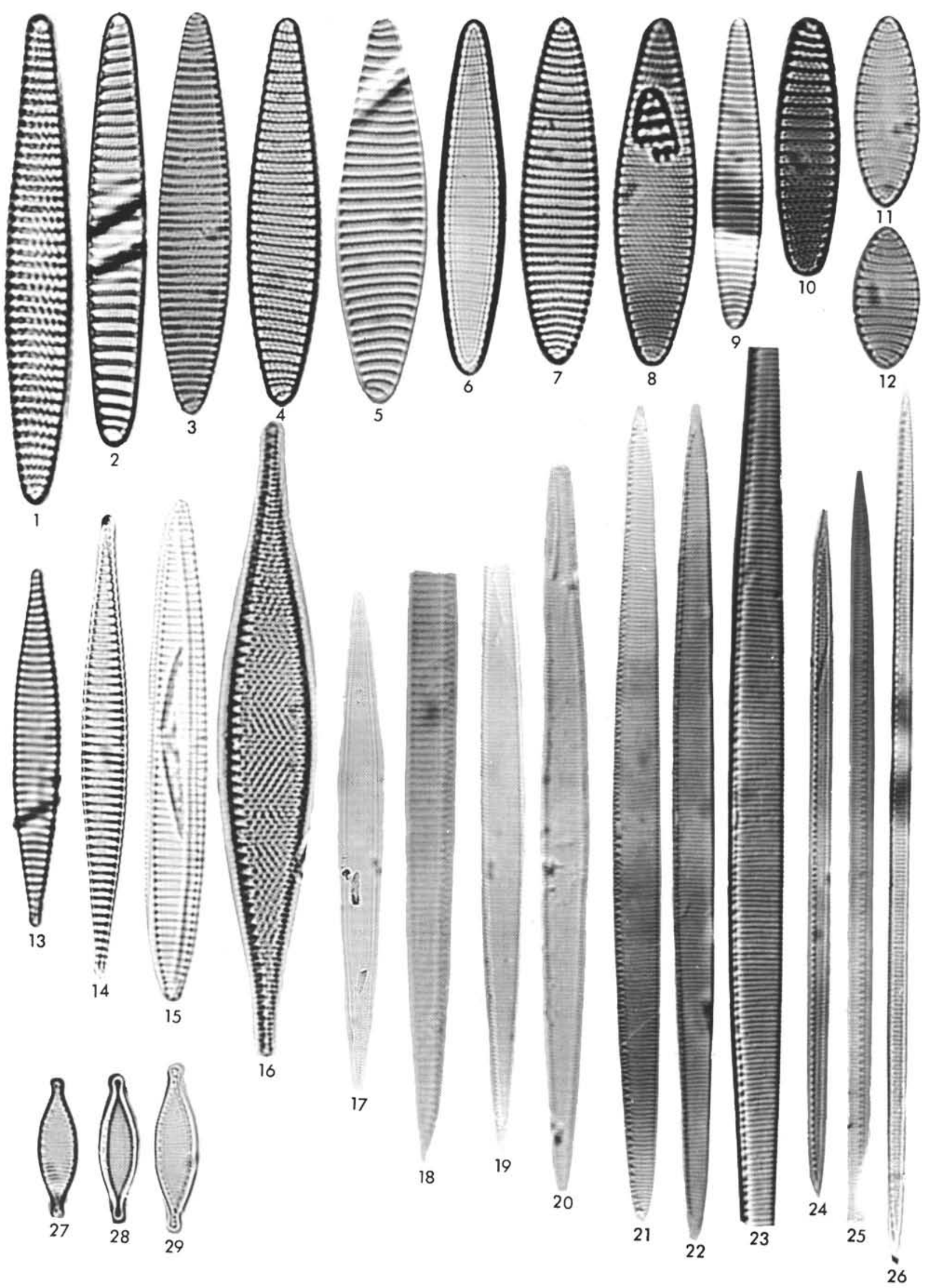




\section{PLATE 4 \\ (Magnification 1500 $\times$ )}

Figures 1-3 Nitzschia turgiduloides Hasle.

1. Station 201.

2. Station 252 .

3. Station 175.

Figure $4 \quad$ Nitzschia turgidula Hustedt. 175.

Figures 5-9 Nitzschia curta (Van Heurck) Hasle.
5. Station 252.
6. Station 252 .
7. Station 252.
8. Station 252.
9. Station 230 .

Figures 10-15 Nitzschia cylindrus (Grunow) Hasle.
10. Station 252.
11. Station 235 .
12. Station 252.
13. Station 307.
14. Station 290.
15. Station 230.

Figure $16 \quad$ Nitzschia vanheurckii (M. Peragallo) Hasle. 235.

Figures 17-19 Asteromphalus hyalinus Karsten.

17. Station 205.

18. Station 235.

19. Station 305.

Figures 20, 21 Asteromphalus parvulus Karsten.

20. Station 305.

21. Station 245.

Figures 22, 23 Asteromphalus heptactis (Bréb.) Ralfs.

22. Station 195.

23. Station 235 .

Figure $24 \quad$ Asteromphalus hookeri Ehrenberg. Station 307. 
PLATE 4
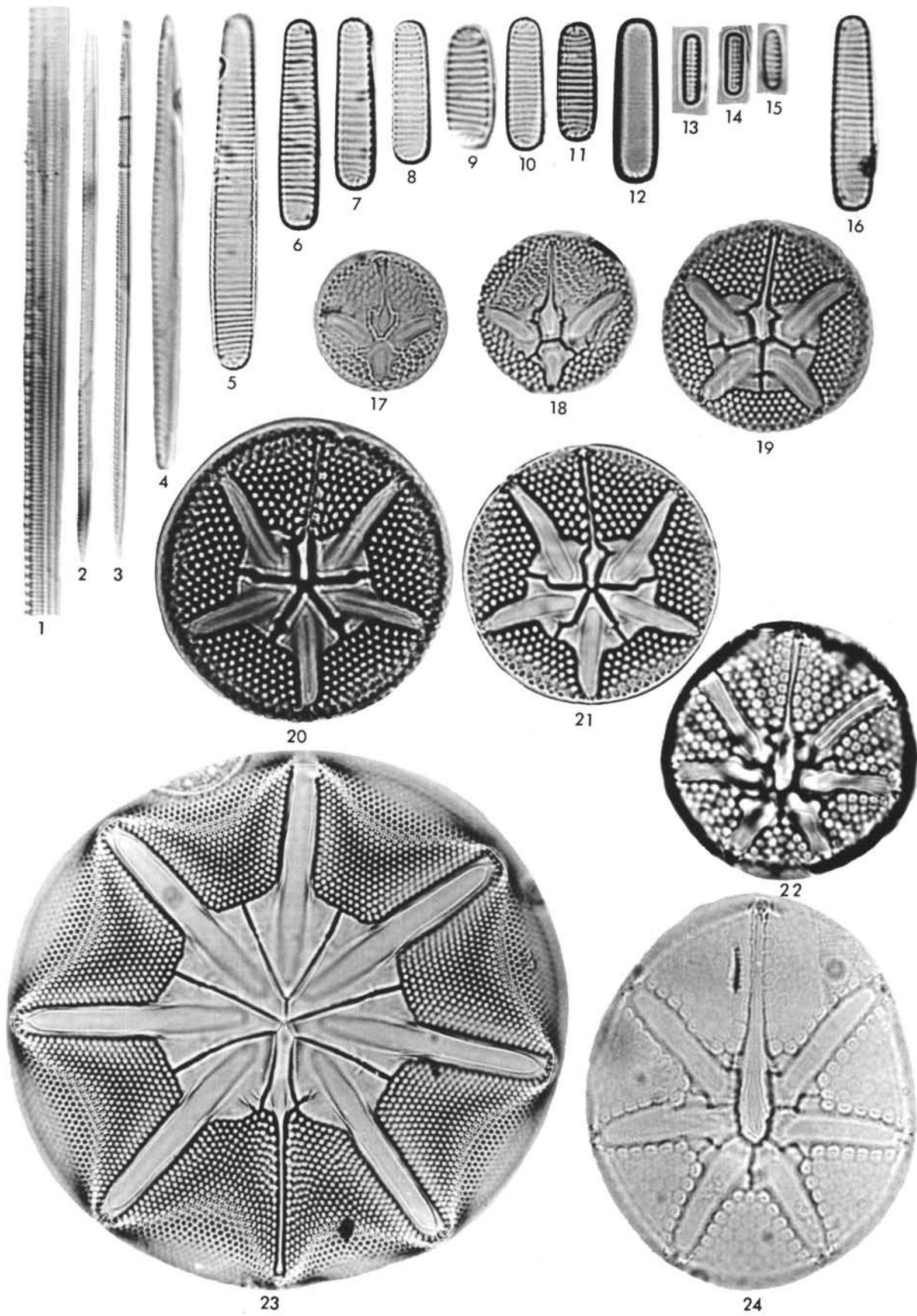
PLATE 5

(Magnification $1500 \times$ )

Figures 1, 2 Actinocyclus ehrenbergii Ralfs. Station 101.

Figures 3,4 Actinocyclus ehrenbergii var. tenella (Bréb.) Hustedt.

3. Station 230 .

4. Station 1.

Figure 5 Charcotia actinochilus (Ehrenberg) Hustedt. Station 252.

Figure $6 \quad$ Lithodesmium undulatum Ehrenberg. Station 1.

Figures 7-9 Eucampia balaustium Castracane.

7. Station 245.

8. Station 170.

9. Station 165 .

Figure $10 \quad$ Actinocyclus ehrenbergii Ralfs. Station 1. 
PLATE 5
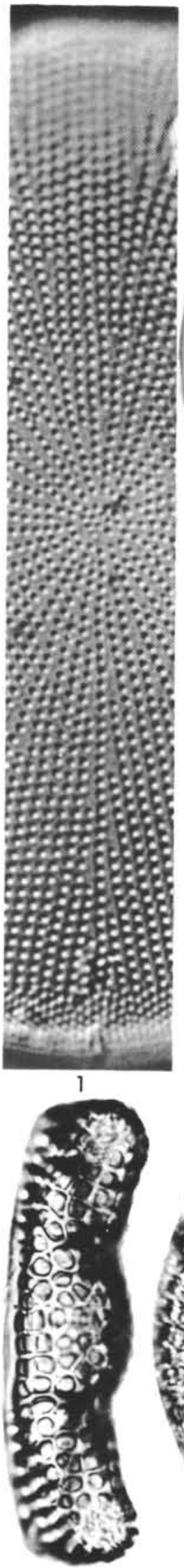

8
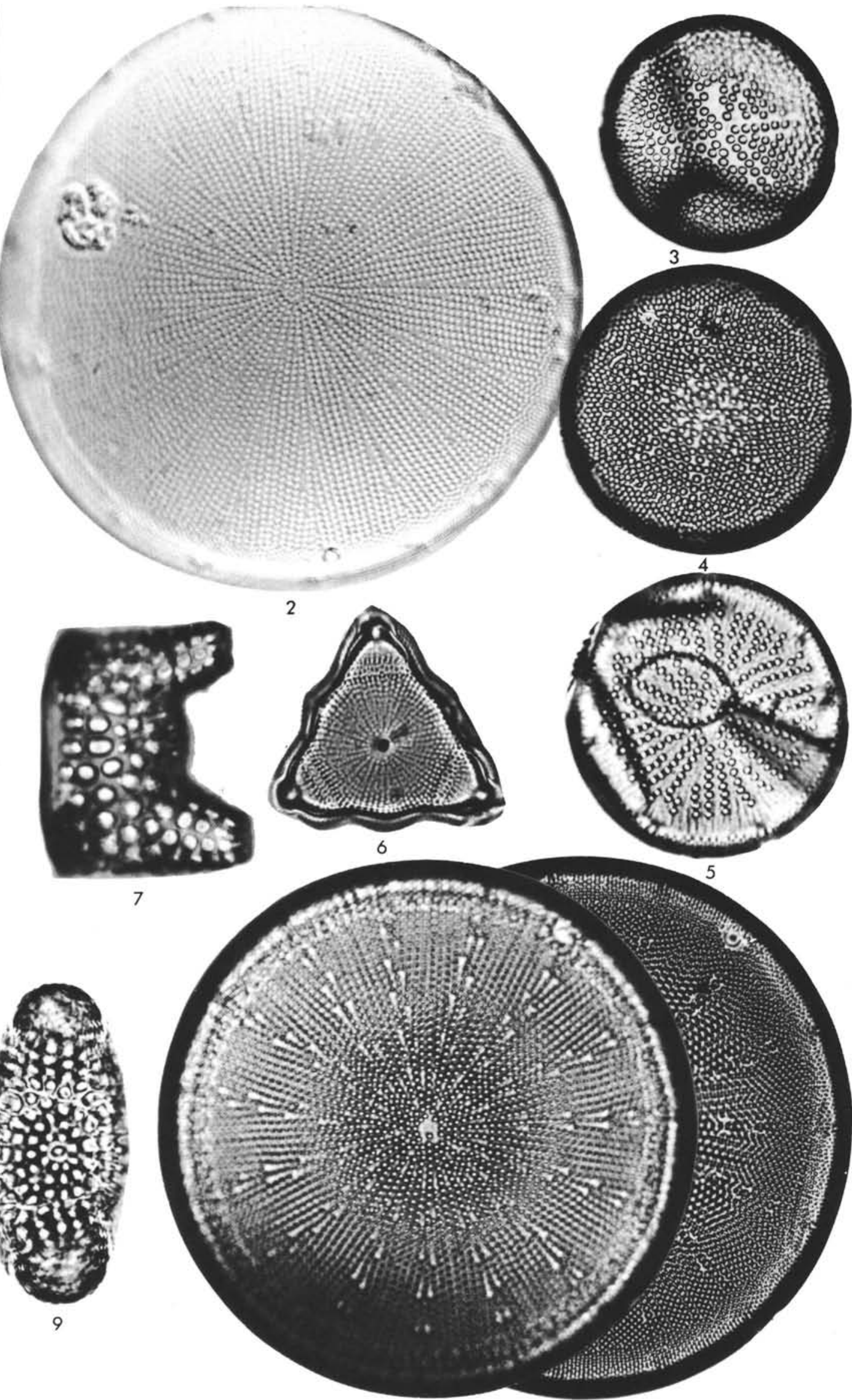
PLATE 6

(Magnification $1500 \times$ )

Figures 1,2 Actinocyclus curvatulus Janisch.

1. Station 75 .

2. Station 105 .

Figures 3-10 Coscinodiscus curvatulus Grunow.

3. Station 185.

4. Station 307.

5. Station 235 .

6. Station 210 .

7. Station 307.

8. Station 195.

9. Station 190.

10. Station 210.

Figures 11-14 Coscinodiscus bullatus Janisch.

11. Station 307.

12. Station 195.

13, 14. Station 307. 
PLATE 6

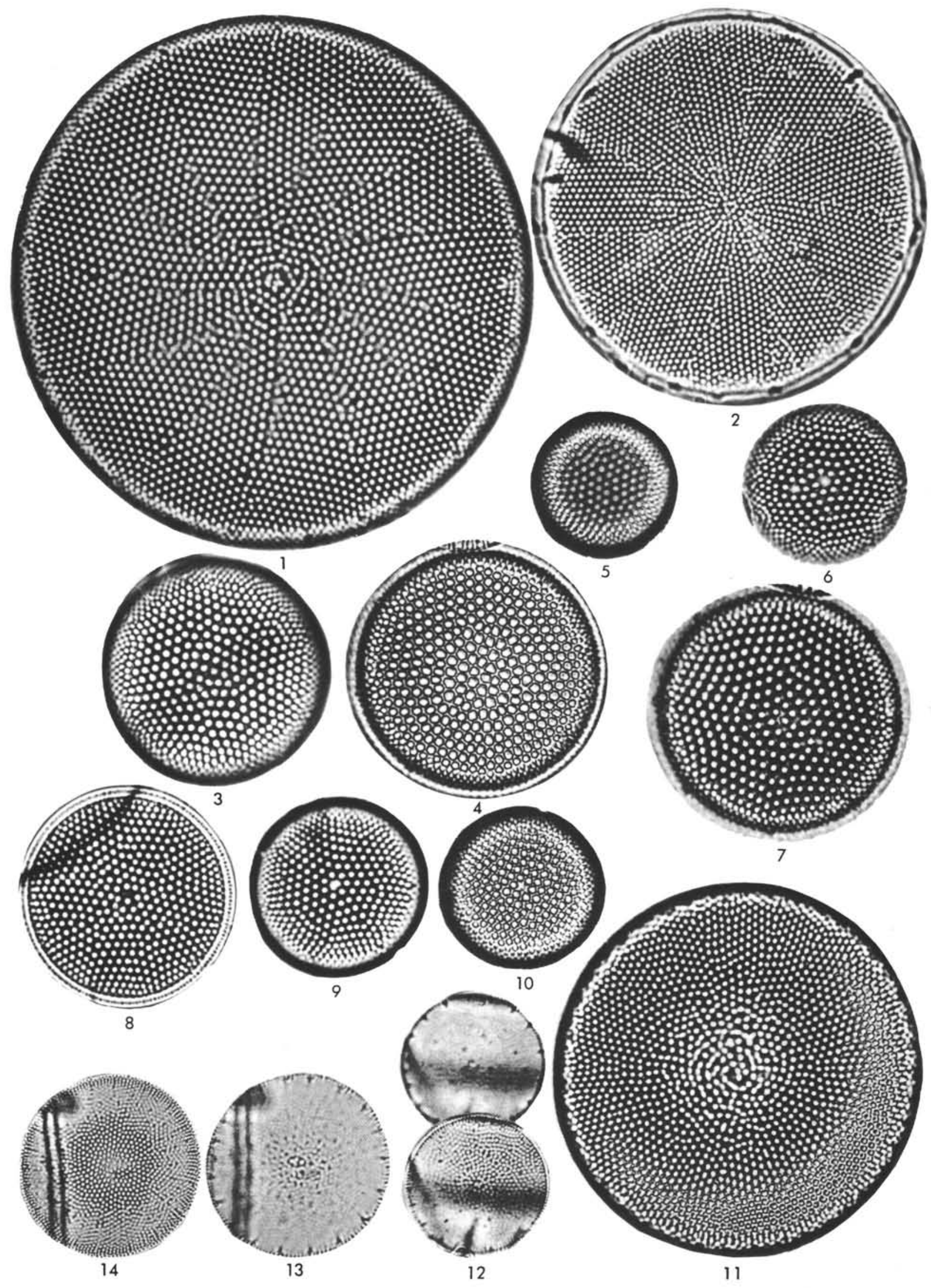


PLATE 7

(Magnification 15-0×)

Figure 1 Coscinodiscus radiatus Ehrenberg. Station 120.

Figure 2 Coscinodiscus kützingi Schmidt. Station 120.

Figure 3 Coscinodiscus lineatus Ehrenberg. Station 305.

Figures 4-6 Coscinodiscus lentiginosus Janisch.

4. Station 235.

5. Station 210.

6. Station 195.

Figure 7 Coscinodiscus crenulatus Grunow. Station 140.

Figures 8,9 Coscinodiscus tabularis var. egregius (Rattray) Hustedt.

8. Station 130.

9. Station 135 .

Figures 10-13 Coscinodiscus tabularis Grunow.

10. Station 205.

11. Station 252.

12. Station 240.

13. Station 307.

Figure 14 Coscinodiscus stellaris var. symbolophorus (Grunow) J $\phi$ rgensen. Station 120. 

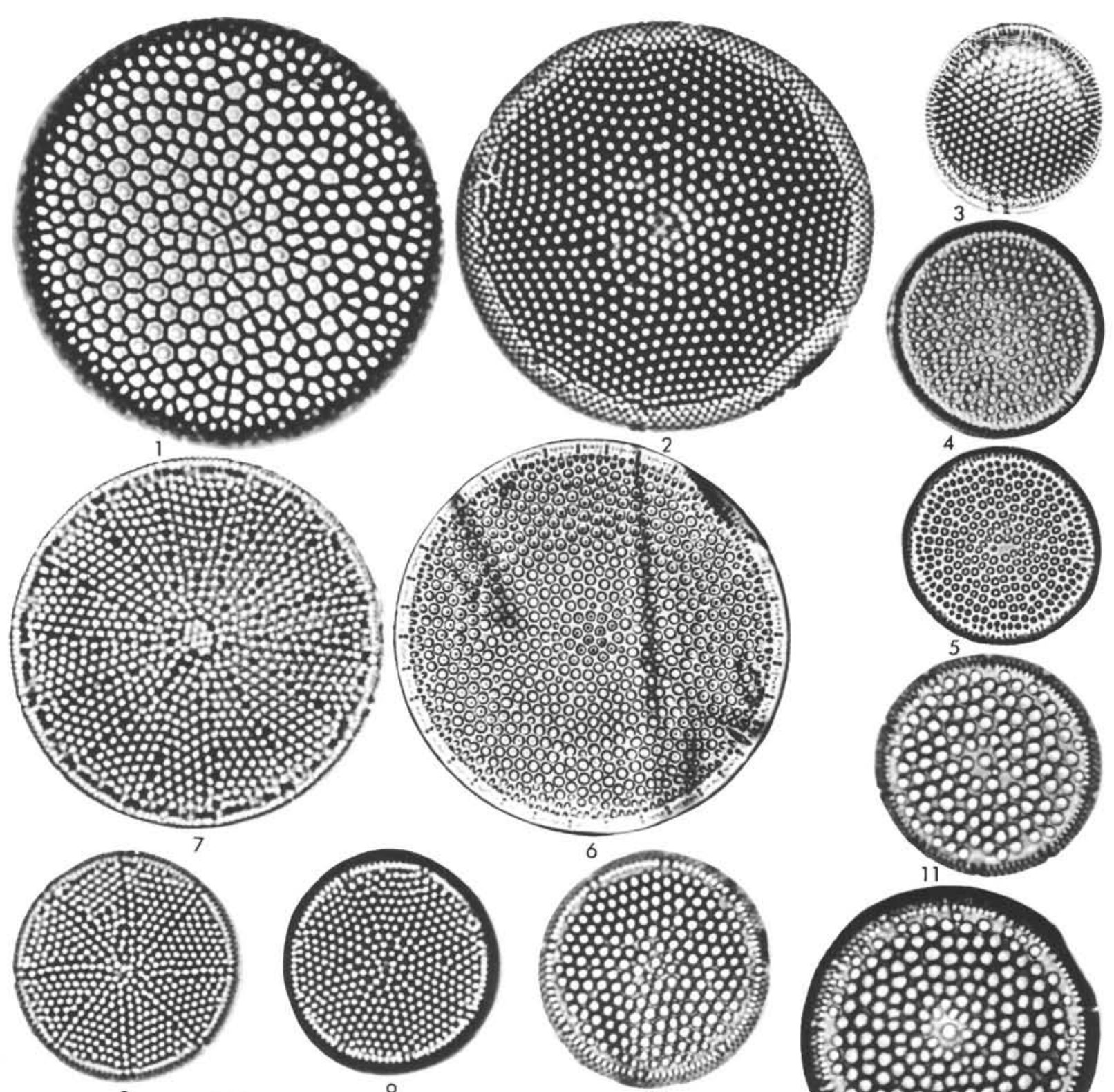

10
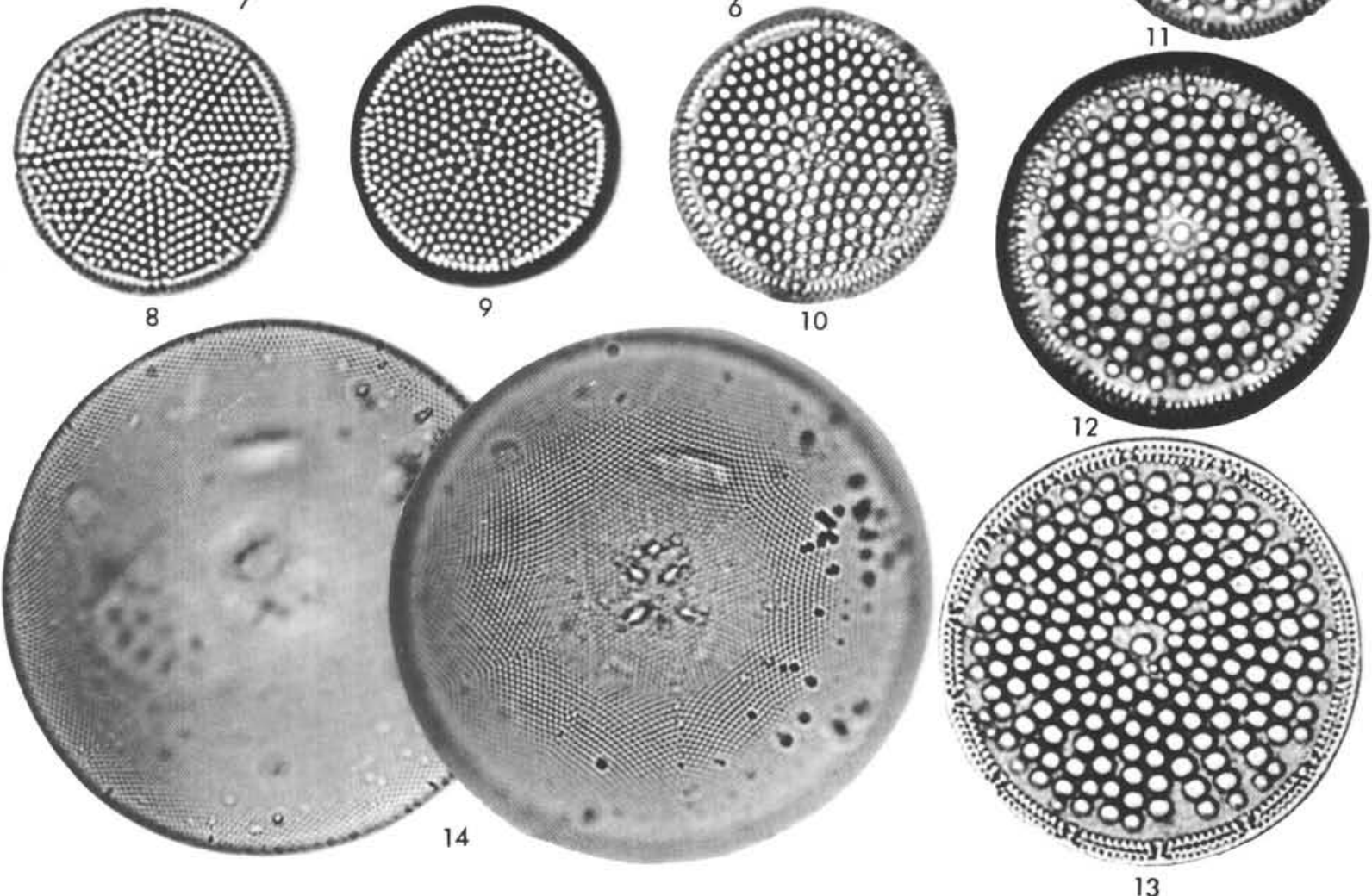


\section{PLATE 8 \\ (Magnification $1500 \times$ )}

Figures 1, 2 Thalassiosira sp. f.

1. Station 201.

2. Station 230 .

Figures 3,4 Thalassiosira sp. g.

3. Station 120.

4. Station 115.

Figure 5 Thalassiosira gravida Cleve. Station 120.

Figures 6,7 Thalssiosira sp. e.

6. Station 300 .

7.Station 235.

Figures 8, 9 Thalassiosira sp. d.

8. Station 120.

9. Station 135.

Figures 10,11 Thalassiosira sp. c. Station 185.

Figures 12-14 Thalassiosira sp. b.

12. Station 135.

13. Station 155.

14. Station 155.

Figures 15-17 Thalassiosira sp. a. Station 270. 
PLATE 8

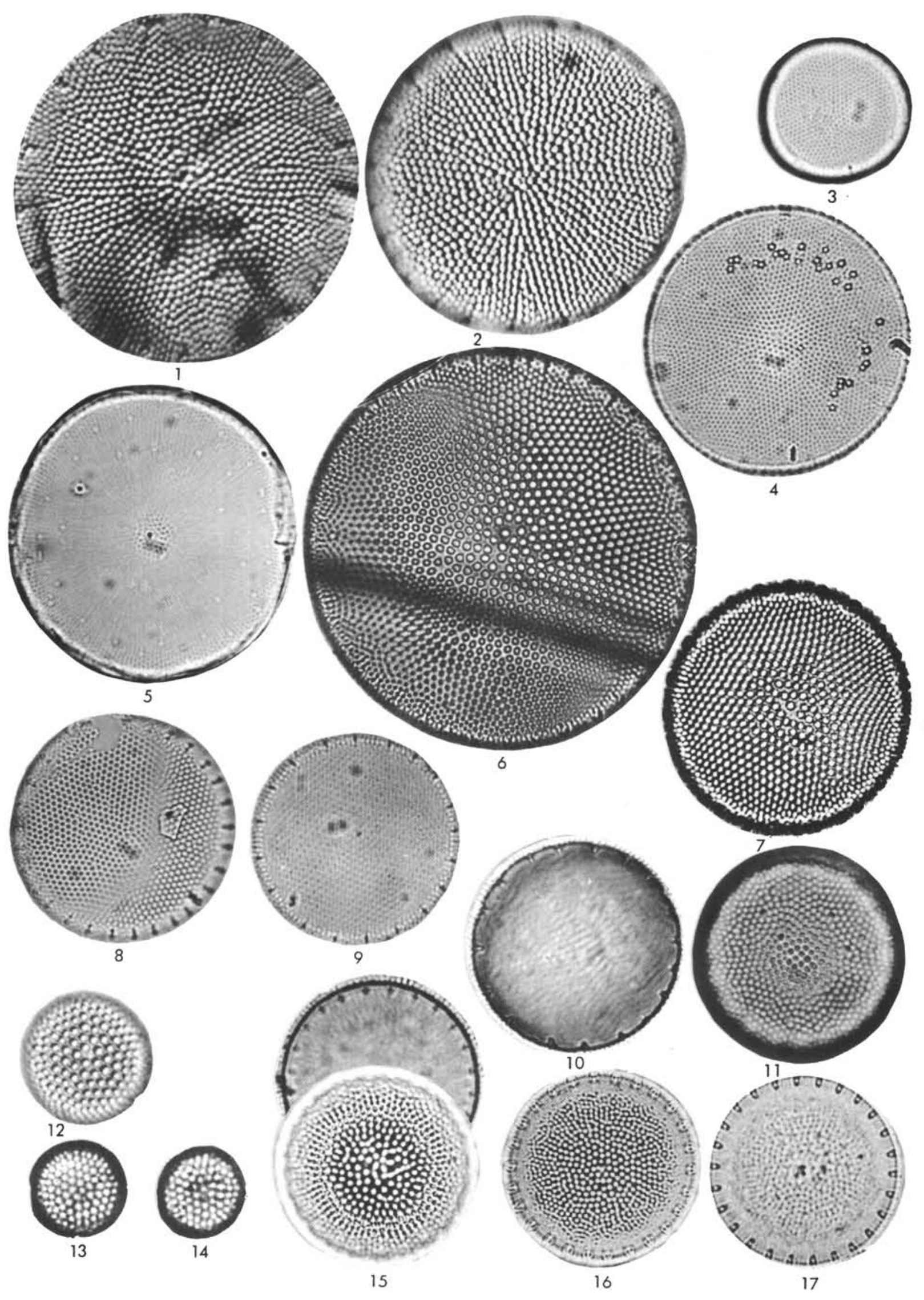




\section{PLATE 9}

(Magnification $1500 \times$ )

Figures 1-5 Thalassiosira oestrupii (Ostenfeld) ProshkinaLavrenko (type 1).

1. Station 185.

2. Station 215 .

3. Station 195.

4. Station 185.

5. Station 240.

Figures 6-10 Thalassiosira oestrupii (Ostenfeld) ProshkinaLavrenko (type 2).

6. Station 205.

7. Station 155.

8. Station 195.

9. Station 190.

10. Station 195.

Figure $11 \quad$ Thalassiosira oestrupii (Ostenfeld) ProshkinaLavrenko (type 1) Station 220.

Figures 12-20 Thalassiosira gracilis (Karsten) Hustedt.

12. Station 300.

13, 14. Station 307.

15. Station 290.

16. Station 305.

17. Station 307.

18. Station 307.

19. Station 307.

20. Station 280 .

Figures 21-25 Thalassiosira delicatula Hustedt.

21. Station 135.

22. Station 270.

23, 24. Station 280 .

25. Station 255 .

Figures 26-31 Thalassiosira poroseriata (Ramsfjell) Hasle (weakly silicified). Station 130 .

Figures 32, 33 Thalassiosira poroseriata (Ramsfjell) Halse (strongly silicified).

32. Station 307.

33. Station 130. 
PLATE 9
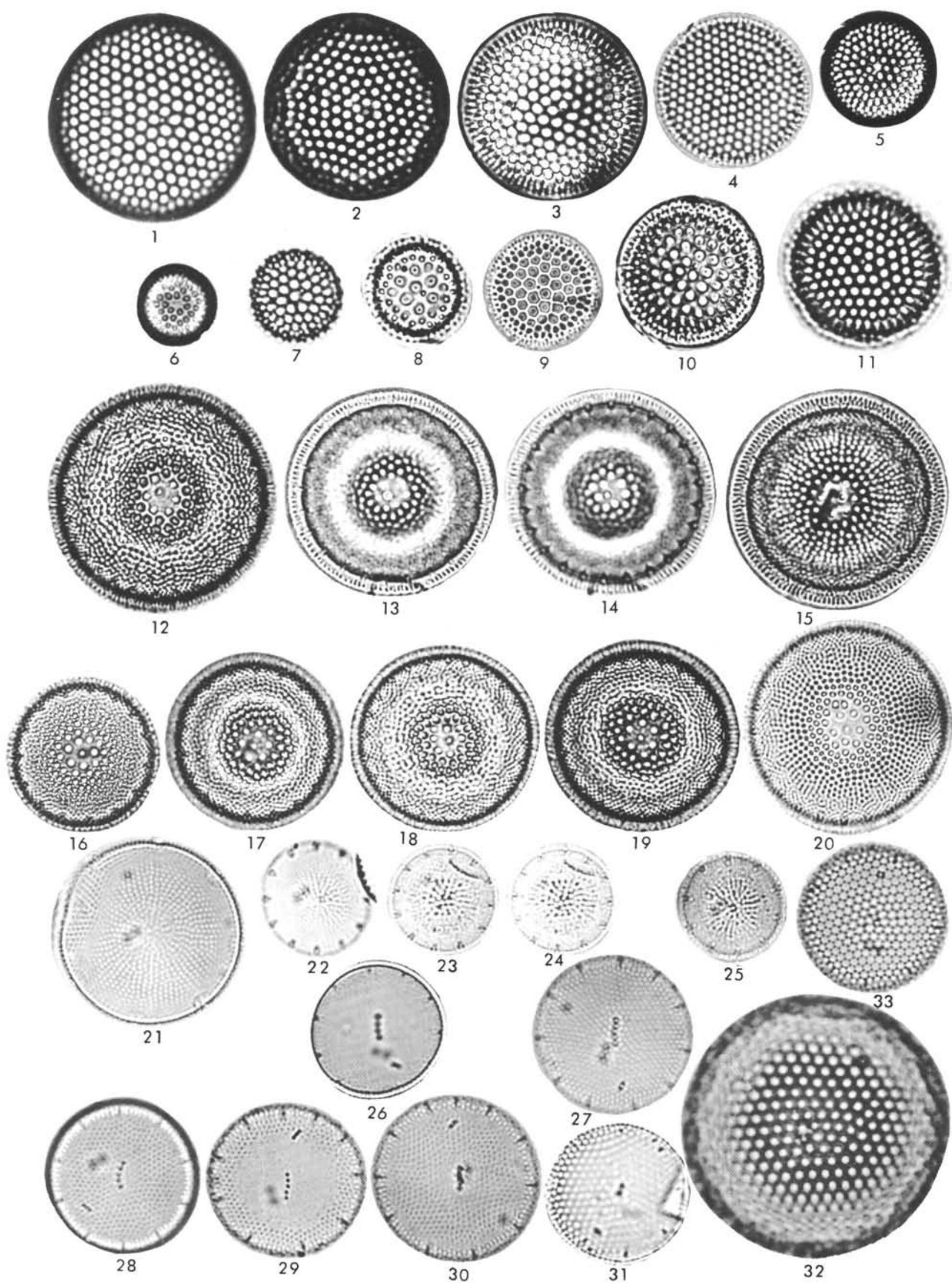
PLATE 10

(Magnification $1500 \times$ )

Figures 1,2 Thalassiosira eccentrica (Ehrenberg) Cleve.

1. Station 220.

2. Station 190.

Figure 3 Thalassiosira eccentrica var. jousé Kanaya. Station 185.

Figures 4, 5 Thalassiosira eccentrica (Ehrenberg) Cleve. Station 75 .

Figures 6,7 Thalassiosira tumida (Janisch) Hasle.

6. Station 120.

7. Station 307. 
PLATE 10
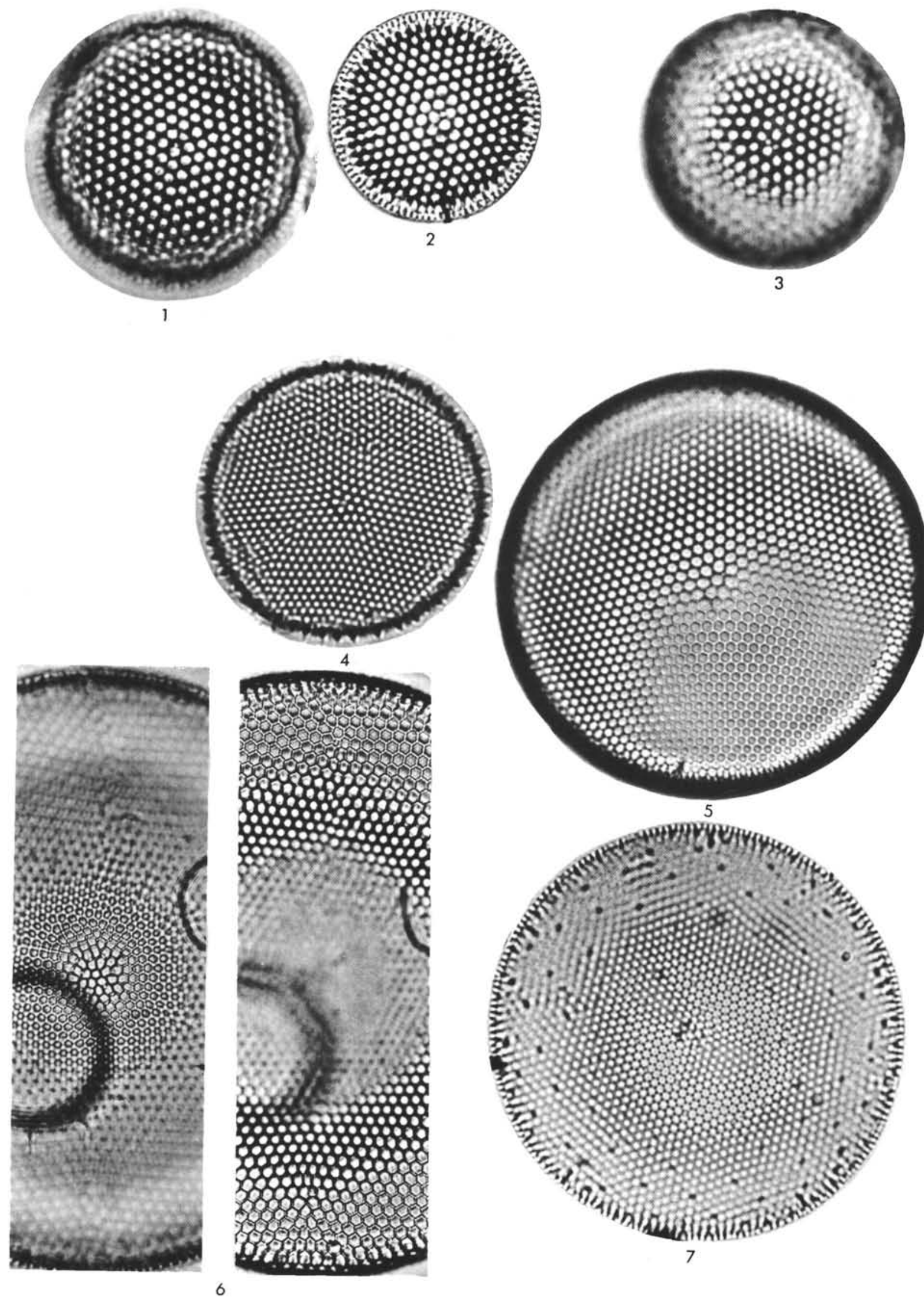


\section{PLATE 11 \\ (Magnification 1500×)}

Figures 1-3 Thalassiosira symmetrica Simonsen. Station 130.

Figures 4-6 Thalassiosira decipiens.

4. Station 50 .

5, 6. Station 170 .

Figure 7 Thalassiosira eccentrica var. jousei Kanaya. Station 235.

Figures 8-10 Thalassiosira lineata Jousé.

8. Station 130 .

9, 10. Station 135 .

Figure 11 Dactyliosolen antarcticus Castracane. Station 145.

Figure 12 Cocconeis costata var. antarctica Manguin. Station 75.

Figure $13 \quad$ Cocconeis aff. placentula Ehrenberg. Station 175.

Figure 14 Amphora 1 (aff. biarcuata Heiden and Kolbe). Station 1.

Figure $15 \quad$ Amphora sp. 2. Station 1.

Figure $16 \quad$ Biddulphia longicruris Greville. Station 1.

Figure 17 Hemidiscus cuneiformis Wallich. Station 140.

Figure $18 \quad$ Biddulphia sp. Station 75. 
PLATE 11
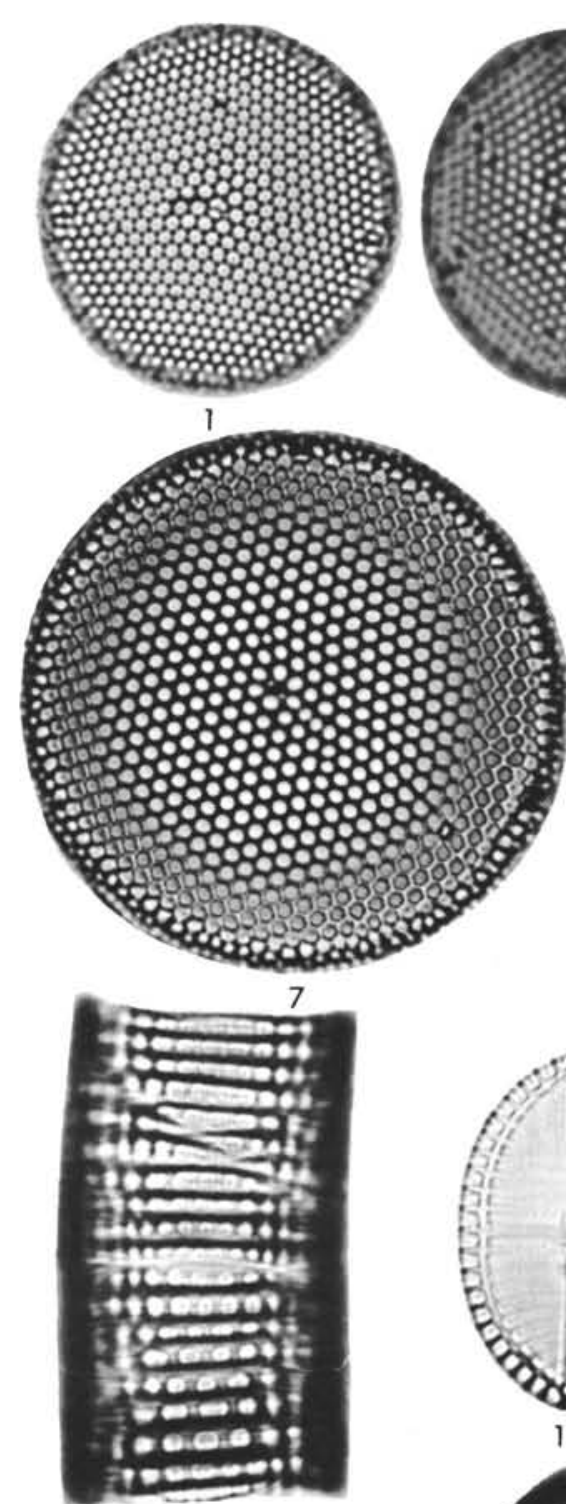

11

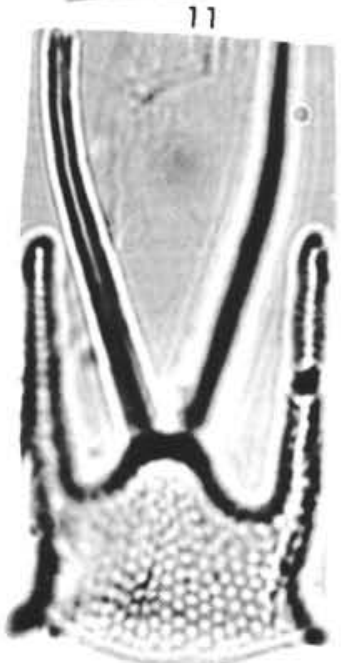

16

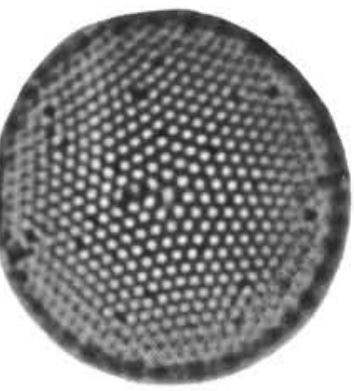

2

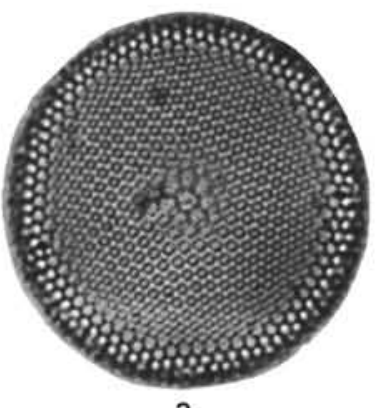

3
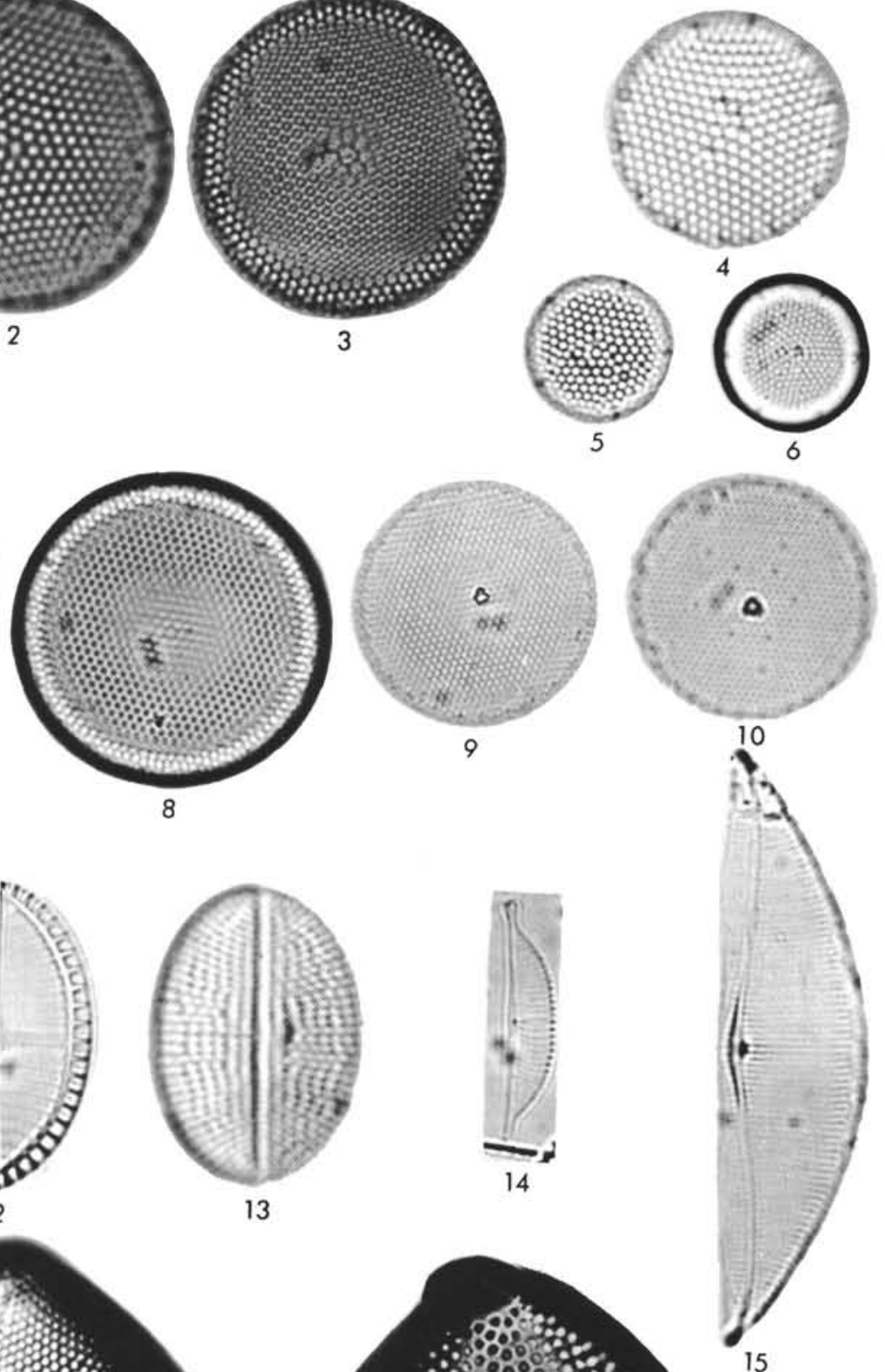
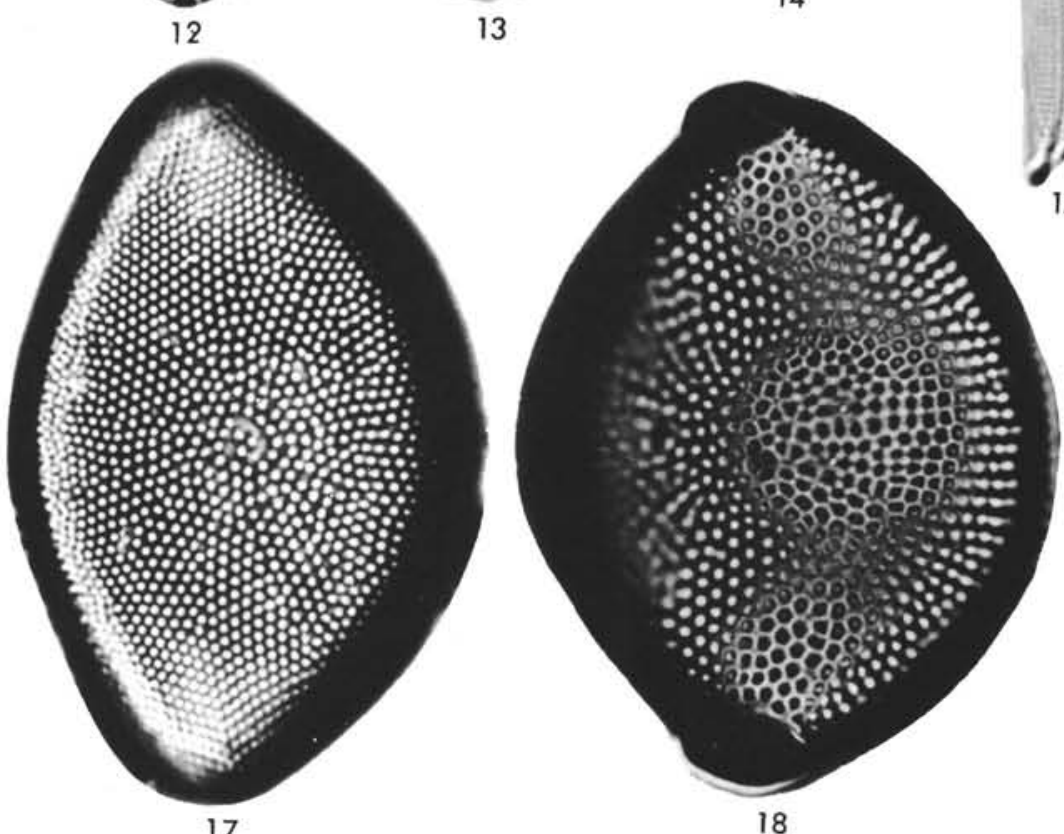
PLATE 12

(Magnification 1500×)

Figures 1-14 Roperia tesselata (Roper) Grunow.

1-6. With coarsely fasciculated, irregularly arranged areolae in the center.

7,8 . With fasciculated areolate structure.

9-14. With tangential areolate structure and 9, 11,12 with oval valves.

1, 2. Station 150.

3. Station 115.

4. Station 140.

5. Station 155.

6. Station 150.

7. Station 101.

8. Station 115.

9, 10. Station 50.

11. Station 135.

12, 13. Station 50 .

14. Station 101 


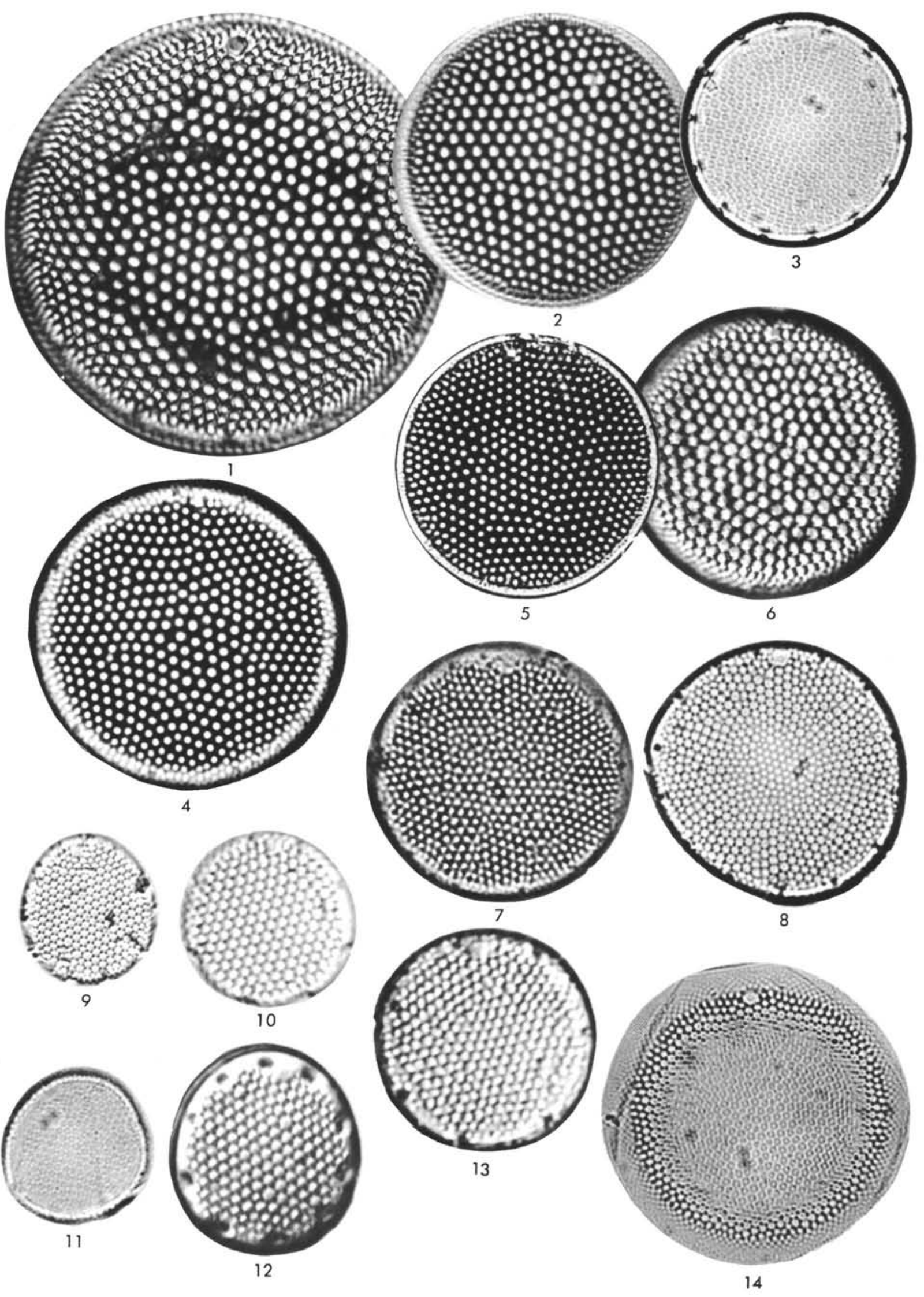


PLATE 13

(Magnification 1500 $\times$ )

Figure $1 \quad$ Rhizosolenia alata Brightwell. Station 50.

Figure 2 Rhizosolenia alata forma inermis (Castracane) Hustedt. Station 195.

Figures 3-5 Rhizosolenia styliformis Brightwell.

3. Station 101.

4. Station 185.

5. Station 180.

Figures 6,7 Rhizosolenia hebetata forma semispina (Hensen) Gran.

6. Station 235.

7. Station 195.

Figure 8 Rhizosolenia hebetata forma bidens Heiden. Station 190.

Figure 9 Rhizosolenia styliformis Brightwell. Station 235.

Figures 10-12 Rhizosolenia hebetata forma hiemalis Gran. 10. Station 220.

11, 12. Station 235 .

Figure 13 Rhizosolenia bergonii Peragallo. Station 50. 
PLATE 13
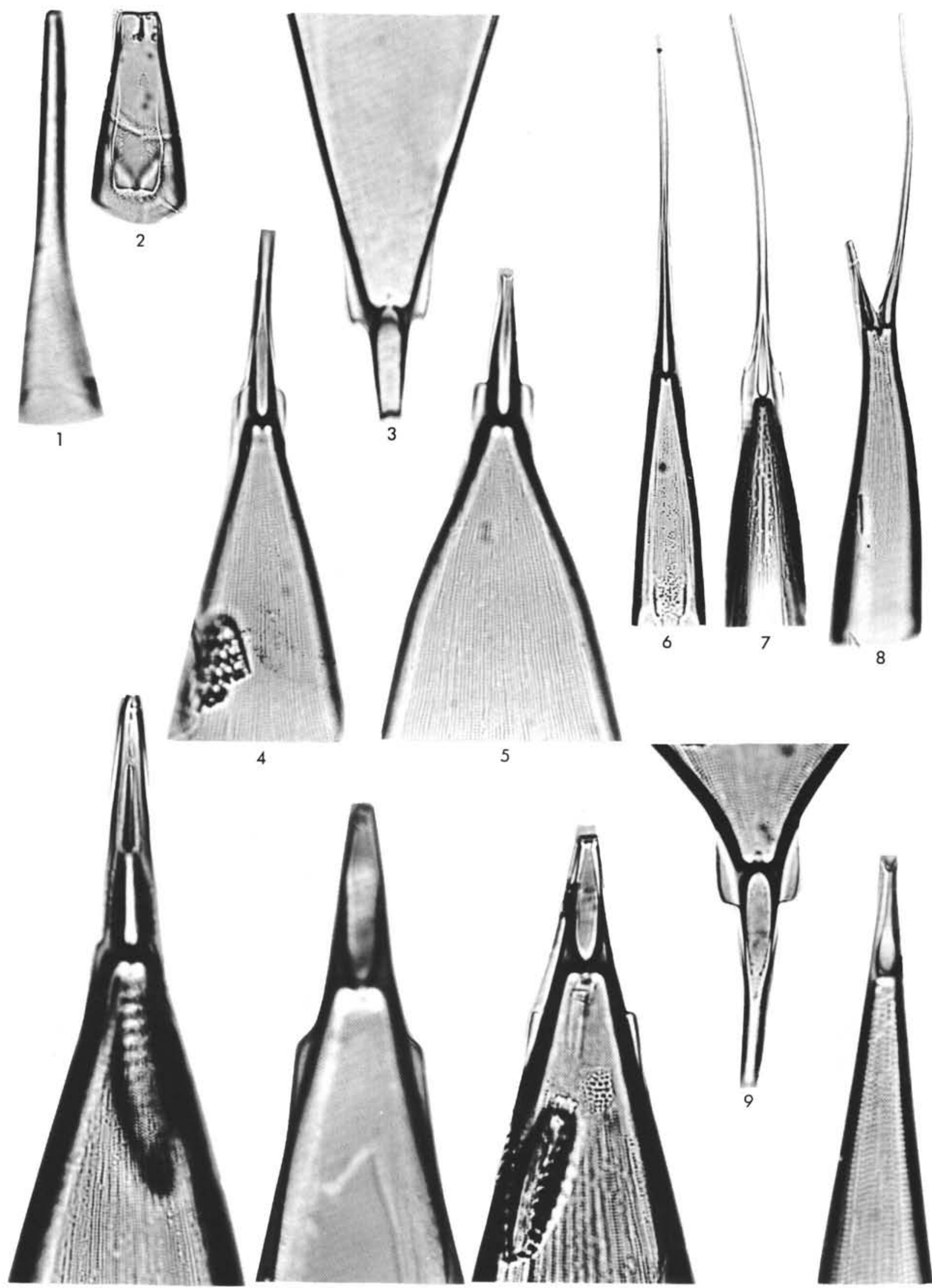

10

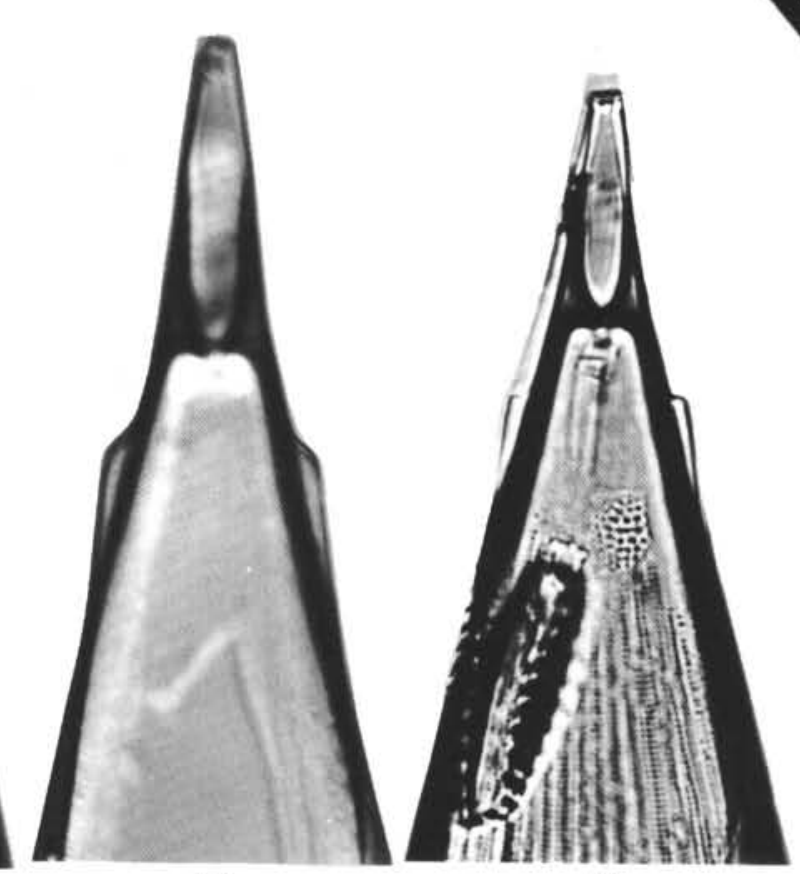

11

12

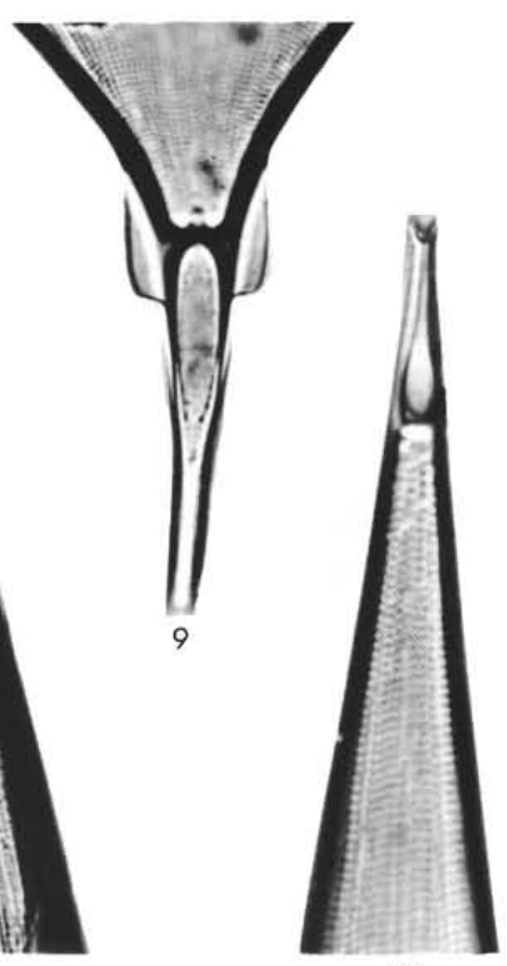

13 
PLATE 14

(Magnification $1500 \times$, except for 6 which is $500 \times$ )

Figures 1-5 Schimperiella antarctica Karsten.

1. Station 185.

2. Station 185 .

3. Station 180 .

4. Station 195.

5. Station 210.

Figure $6 \quad$ Pleurosigma directum Grunow. Station 205.

Figure $7 \quad$ Navicula directa (W. Smith) Ralfs. Station 175.

Figure $8 \quad$ Tropidoneis antarctica (Grunow) Cleve. Station 1.

Figure $9 \quad$ Navicula trompii Cleve. Station 290.

Figure 10 Thalassionema nitzschioides var. parva Heiden. Station 50.

Figure 11 Thalassionema nitzschioides Grunow aberrant form. Station 190.

Figure 12 Pseudoeunotia doliolus (Wallich) Grunow. Station 1.

Figure 13 Trachyneis aspera (Ehrenberg) Cleve. Station 101. 
PLATE 14
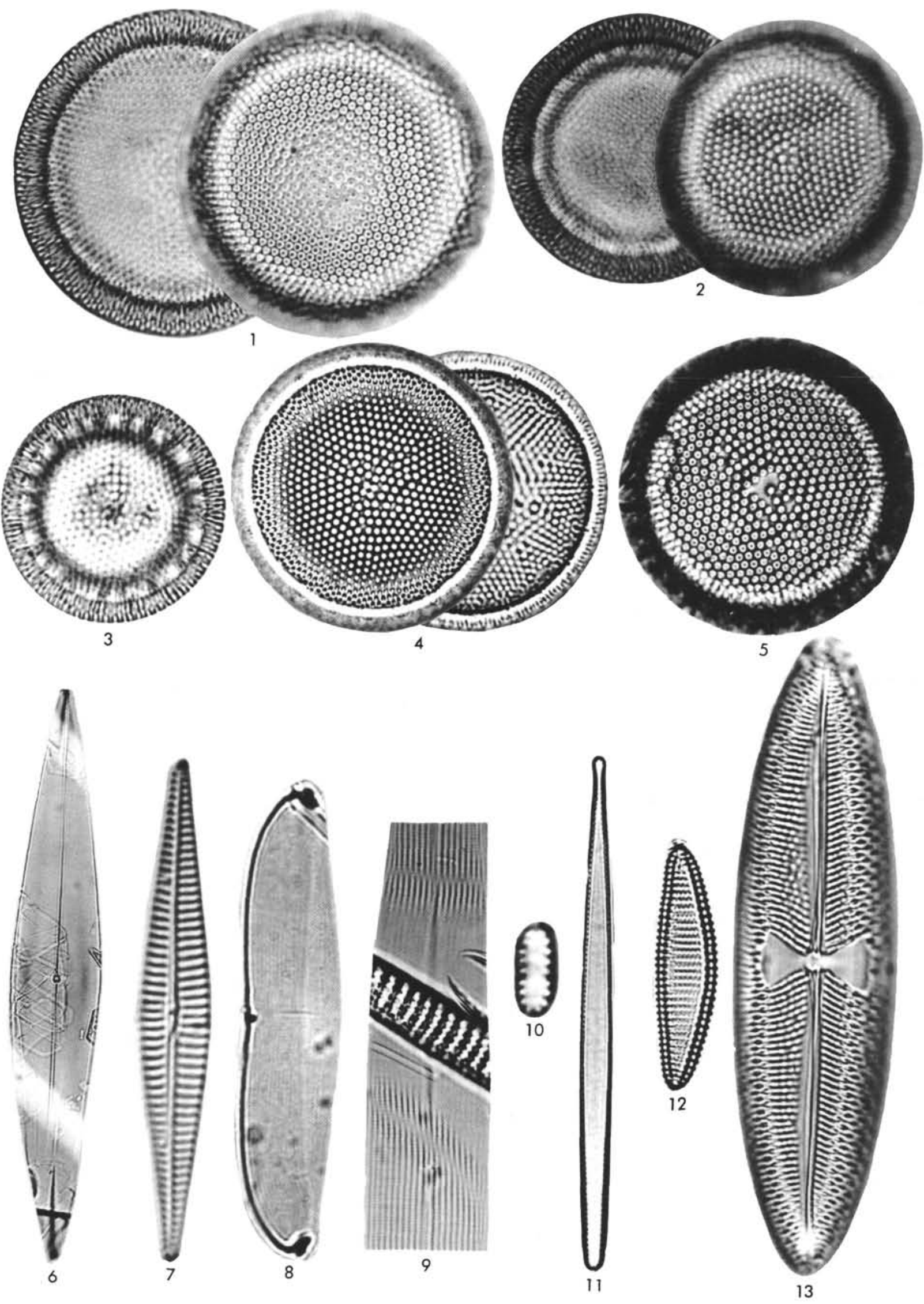